\title{
Phase Space Theory of Bose-Einstein Condensates and Time-Dependent Modes
}

\author{
B J Dalton* \\ ARC Centre for Quantum-Atom Optics \\ and \\ Centre for Atom Optics and Ultrafast Spectroscopy \\ Swinburne University of Technology \\ Melbourne, Victoria 3122, Australia
}




\subsection{Abstract}

A phase space theory approach for treating dynamical behaviour of Bose-Einstein condensates applicable to situations such as interferometry with BEC in timedependent double well potentials is presented. Time-dependent mode functions are used, chosen so that one, two,.. highly occupied modes describe well the physics of interacting condensate bosons in time dependent potentials at well below the transition temperature. Time dependent mode annihilation, creation operators are represented by time dependent phase variables, but time independent total field annihilation, creation operators are represented by time independent field functions. Two situations are treated, one (mode theory) is where specific mode annihilation, creation operators and their related phase variables and distribution functions are dealt with, the other (field theory) is where only field creation, annihilation operators and their related field functions and distribution functionals are involved. The field theory treatment is more suitable when large boson numbers are involved. The paper focuses on the hybrid approach, where the modes are divided up between condensate (highly occupied) modes and non-condensate (sparsely occupied) modes. It is found that there are extra terms in the Ito stochastic equations both for the stochastic phases and stochastic fields, involving coupling coefficients defined via overlap integrals between mode functions and their time derivatives. For the hybrid approach both the Fokker-Planck and functional Fokker-Planck equations differ from those derived via the correspondence rules, the drift vectors are unchanged but the diffusion matrices contain additional terms involving the coupling coefficients.

Results are also presented for the combined approach where all the modes are treated as one set. Here both the Fokker-Planck and functional Fokker-Planck equations are exactly the same as those derived via the correspondence rules. However, although the Ito stochastic field equations are also unchanged, the Ito equations for the stochastic phases contain an extra classical term involving the coupling coefficients.

* Tel: +61 039214 8187; fax: +610392145160

Email address: bdalton@swin.edu.au 


\section{Introduction}

Bose-Einstein condensates (BEC) in cold atomic gases are a quantum system on a macroscopic scale. At temperatures well below the critical temperature almost all the bosonic atoms occupy the same single particle state or mode 11. Consequently, BEC exhibit coherence properties analogous to those for an optical laser where macroscopic occupancy of photons in a single mode can occur, and therefore their application for interferometry was a logical outcome following early experiments demonstrating that BEC could show interference effects [2, 3. However, unlike the photons in a laser, the bosonic atoms in a BEC interact with each other and this can cause decoherence effects that degrade interference patterns. Ideally, the BEC should be at a temperature well below the transition temperature, so that coherence is enhanced due to all the bosons occupying only one or two modes, with decoherence effects due to thermally occupied excited modes becoming small. However such low temperatures are not always realised. The interactions between the bosons could in principle be made zero via Feshbach resonance methods [4, but this is hard to accomplish over the range of trapping magnetic fields involved during an interferometric process and requires a broad Feshbach resonance. Furthermore, it is bosonic interactions that provide for the possibility of realising Heisenberg limited interferometry via spin squeezing effects [5], [6, and proposals for such interferometry have been made [7, 8] in which the bosonic interactions are central. Recent reviews of BEC interferometry include [9, 10, 11, 12.

The theoretical treatment of BEC interferometry must take into account the many body nature of the BEC including the presence of boson-boson interactions. The interferometry process is time dependent and may involve non-static trapping potentials, such as when the trap changes from a single well to a possibly asymmetric double well and back again. The possibility of fragmentation of the BEC into more than a single mode [1] must be allowed for, since it cannot just be assumed that all bosons stay in one single condensate mode throughout the process even if this was initially the case. Decoherence effects associated with bosons being lost from condensate modes need to be treated, including the creation of Bogoliubov excitations [13. Unless the BEC is prepared well below the transition temperature thermal effects would also need to be taken into account. Also, the experiments may not control the total number of bosons in the BEC. These considerations suggest that the quantum state of the BEC should be described via a density operator rather than a pure state, and the many body nature of the BEC would best be discussed in terms of field operators and second quantisation rather than via standard first quantisation methods involving symmetrised products of wave functions.

A number of different theoretical approaches have been used for studying BECs, including treatments using variational methods, Heisenberg equations, master equations and phase space distributions. General descriptions of and references to these methods are set out in [14. The present paper focuses on

phase space methods which involve the overall bosonic field rather than just the separate modes - these were originally developed for applications in quantum 
optics [15], 16], 17], [18, [19], but are now applied in quantum-atom optics [20, 21. The quantum density operator is represented either by a quasi-distribution function in a phase space involving variables that replace the mode annihilation, creation operators for treatments where it is practical to consider separate modes, or by a quasi-distribution functional in a phase space involving field functions which replace the bosonic field annihilation, creation operators. As these two situations are interconvertible it is convenient to consider both though ultimately the paper is focused on the latter. Also, it is often convenient to define field operators for condensate and non-condensate modes separately [22], [23, and for situations well below the transition temperature often only one or two condensate modes are involved. Quantum correlation functions involving products of annihilation, creation operators either for the separate modes or for the bosonic field are given by phase space ordinary or functional integrals of the equivalent products of phase space variables or field functions, weighted by the quasi-distribution function or functional. Furthermore, there are several possible choices for the type of quasi-distribution function or functional that may be used. The positive $\mathrm{P}$ type is suitable for consideration of quantum correlation functions involving normally ordered products of annihilation, creation operators, the Wigner type is better suited to treat those that involve symmetrically ordered products. Indeed, when the BEC is well below the transition temperature a hybrid approach, such as where the highly occupied condensate modes are described via a Wigner quasi-distribution function or functional and the positive $\mathrm{P}$ type is used for the mainly unoccupied non-condensate modes [20, 24], 25], 14, might best describe the physical situation where the condensate bosons behave essentially like a classical mean field and the non-condensate bosons exhibit mainly quantum features . In the phase space approaches the Liouvillevon Neumann or master equation for the density operator is replaced by either Fokker-Planck or functional Fokker-Planck equations for the quasi-distribution function or functional. Finally, the Fokker-Planck equations are replaced by equivalent Ito stochastic equations for either stochastic phase space variables or stochastic fields, and the phase space ordinary or functional integrals for the quantuim correlation functions given by stochastic averages.

Phase space methods first involve a consideration of what are suitable single particle states or modes that could be used to describe states of the bosonic system. In treating both the dynamics of bosonic systems and their static behaviour at non-zero temperatures it is usually convenient to use Fock states as a basis. In first quantization the Fock states are symmetrised products where the identical bosons occupy single particle states or modes, and for bosons there are Fock states in which more than one particle can occupy any particular mode. In an idealised Bose-Einstein condensate of non-interacting particles at zero temperature all $N$ bosons occupy the mode with lowest single particle energy. Finite temperature effects for non-interacting bosons allow for excitations to higher energy modes. Below a critical temperature $T_{c}$ Bose-Einstein condensation occurs as a phase transition. For non-interacting bosons in a static potential $V$ the usual choice for the single particle states is the time independent energy eigenstates for a single boson in the static potential. However, for interacting bosons 
in cases where the trap potential $V$ changes with time - such as in interferometry experiments involving Bose-Einstein condensates - there is no obvious set of time independent modes that could be used. Of course theoretical treatments using time independent modes can still be carried out, often these are chosen for mathematical convenience such as in facilitating numerical calculations. For example Blakie et al 26] use single particle states for non-interacting bosons in a static trap potential, Egorov et al [27] use plane wave states. It is well known however that for interacting bosons at temperatures close to zero, the solution to the time dependent Gross-Pitaevskii equation [28, [29] usually provides a good physical choice for the single particle state that all the bosons occupy. This mode function is obtained from the Dirac-Frenkel variational principle [30, 31] in which the state vector is a product with all bosons occupying the same single particle state. However, as Leggett has pointed out [1 BoseEinstein condensates may sometimes exist in fragmented states, in which there is macroscopic occupancy of more than one mode. Such a situation could occur in double-well interferometry with Bose-Einstein condensates, where two modes may be involved. These may be two localised modes in the separate potential wells, or they may be delocalised modes spread over both wells, in a symmetric double well one could be symmetric and the other antisymmetric. Generalised coupled Gross-Pitaevskii equations [32, 33, 34, 35, [36, [37, 38, [14, 39] can be obtained via the Dirac-Frenkel variational principle for the actual pair of mode functions. Theories in which all the bosons occupy only or a few single particle states where the mode functions satisfy Gross-Pitaevskii type equations are referred to as mean field theories, since the Gross-Pitaevskii type equations contain non-linear terms which can be interpreted as due to mean fields. However, the actual dynamics is more complicated than that can be treated via mean field theories, processes associated with unoccupied modes such as Bogoliubov excitations [40, 1], 13. need to be considered. Phase space theories are a standard approach to moving beyond mean field theories. Since such time dependent modes or single particle states provide a useful first approximation to treating the dynamics of interacting bosons in time dependent potentials at temperatures well below $T_{c}$, it is natural to base the phase space theory on time dependent modes as these more closely describe the BEC before interactions involving both occupied and non-occupied modes are included. It is also possible that choosing time-dependent modes could facilitate numerical calculations. A redevelopment of phase space theory to allow for this modal time dependence is warranted.

However, if the theory is to be based on time dependent modes it follows that as the total bosonic field annihilation, creation operators are not time dependent, then the mode annihilation, creation operators must be. Since in phase space theories these mode annihilation, creation operators are represented via the phase space variables, the question arises as to whether these should be chosen to be time dependent to reflect the time dependence of the mode functions. It turns out that although the phase space theory can also be developed in terms of using time independent phase space variables to represent the time dependent mode annihilation, creation operators, the field functions that represent the to- 
tal bosonic field annihilation, creation operators via mode expansions with the phase space variables as coefficients then become time dependent. This is rather unsatisfactory as it would be preferable to represent time independent bosonic total field operators by time independent field functions. There are other complications with using time independent phase space variables, so the present paper is framed around using phase space variables whose time dependence is related to the time dependence of the mode functions. The time dependence of the phase space variables is actually chosen to be identical to that for the mode annihilation, creation operators. Both the Wigner and positive $\mathrm{P}$ distribution functions or functionals are treated, both in a double phase space. The theory presented here treats two situations, one (mode theory) being where mode annihilation, creation operators and their related phase variables and distribution functions are dealt with specifically, the other (field theory) being where field creation, annihilation operators and their related field functions and distribution functionals involve a description where individual modes are not distinguished.

A so-called combined approach could be adopted, in which the modes are not divided into condensate and non-condensate types, either when separate modes are considered or in the field theory treatment involving the total fields. However, even in the combined approach there are some unexpected consequences. For the phase space distribution functions involved in the mode theory situation, although the Fokker-Planck equations and the results for the quantum correlation functions as averages via the phase space integrals are unchanged from those for the case of conventional time independent mode functions, the relationship between the Ito stochastic equations and the Fokker-Planck equations now includes an additional classical term that depends on the time dependence of the mode functions via the so-called coupling coefficients. These are defined via integrals of products of mode functions with the mode function time derivatives. On the other hand, when the field theory results are derived, not only are the functional Fokker-Planck equations for the phase space distribution functional and the results for the quantum correlation functions as averages involving phase space functional integrals unchanged from those for the situation of conventional time independent mode functions, but the relationship between the Ito stochastic field equations and the functional Fokker-Planck equations no longer includes any additional term that depends on the time dependence of the mode functions. These results confirm the validity of phase space distribution functional results for functional Fokker-Planck equations and Ito stochastic field equations presented elsewhere [20, 26] in the situation where the total bosonic fields are considered.

The situation changes for the so-called hybrid approach [20, 24, 25, 14], which is the approach treated in the present paper. In the hybrid approach, the modes are divided into the highly occupied condensate modes and the sparsely occupied non-condensate modes, these being treated in terms of a Wigner or positive $\mathrm{P}$ distribution functions or functionals respectively. The total bosonic field operators are the sum of condensate and non-condensate field operators. As in Refs 24, 14] time dependent mode functions are involved in both cases, and the condensate and non-condensate field operators now also become time 
dependent. Hence the field functions that represent them in the phase space theory are no longer time independent, as was the case for the total fields. The phase space theory is therefore more complex than that outlined previously in 14, both when separate modes are considered and when condensate and noncondensate fields are involved. However, in both the mode and field theory situations, ordinary or functional Fokker-Planck equations and Ito stochastic equations for the phase space variables or fields can still be derived, and relationships between the drift, diffusion terms in the Fokker-Planck equations and the classical and noise terms in the Ito stochastic equations established both for separate phase variables and for the fields. Hybrid Wigner and positive $\mathrm{P}$ distribution functions or functionals are treated, both in a double phase space. Both the ordinary and functional Fokker-Planck equations now have additional diffusion terms involving coupling coefficients between condensate and non-condensate modes. The drift terms are unchanged. Also, additional classical terms involving the coupling coefficients need to be included in the relationship between the Ito stochastic (field) equations and the (functional) Fokker-Planck equation, and the noise terms are now related to the new diffusion term. These additional terms modify the functional Fokker-Planck and Ito stochastic field equations presented previously in Ref. [14]. We show that the condensate and non-condensate stochastic fields involve the same expansions in terms of time dependent stochastic phases and mode functions as do the condensate and non-condensate field functions when expanded in terms of non-stochastic phase variables.

The theory for the hybrid approach is presented in two parts, the first (mode theory) dealing with separate time dependent modes, their annihilation and creation operators, phase variables, the mode quantum correlation functions, characteristic and distribution functions, Fokker-Planck and Ito stochastic equations for the phase variables associated with separate mode annihilation, creation operators. This more familiar separate mode theory treatment will be covered in Section 2 for the hybrid approach. In the second part (field theory) we consider field annihilation and creation operators, field functions, field correlation functions, characteristic and distribution functionals, functional Fokker-Planck and Ito stochastic equations for the fields associated with condensate and noncondensate field annihilation, creation operators. This less familiar quantum field theory treatment will be covered in Section 3 for the hybrid approach where the total fields are divided into condensate and non-condensate fields. However, although the quantum field theory treatment is more useful for dealing with systems containing a large number of bosons, it is less familiar than the separate mode approach, involving as it does concepts based on functional calculus. Consequently the key steps for the quantum field theory treatment will be set out as a separate entity, rather than just deriving the functional Fokker-Planck and Ito stochastic field equations from the previous separate mode results. Connections between the separate mode and quantum field theory treatments will be frequently emphasised however. Both in the separate mode and the field theory treatments the stochastic equations are the sum of a classical contribution related to the drift vector and a noise contribution involving Gaussian-Markov 
random noise terms. The stochastic properties of the noise contributions are derived and related to the diffusion matrices. The conclusions are presented in Section 4, along with a comparison to phase space theories based on time independent modes. Key results are summarised in Section 5 both for the hybrid approach and for the combined approach. In Appendix 6 a brief outline of functional calculus is presented, and the equivalence of the separate mode and field theory treatments is shown in Appendix 7 for the hybrid approach. The paper is also accompanied by several further Appendices, available as On-line Supplementary Material. Appendices 8, 9] and10 include details of derivations for Sections 2 and 3 to avoid overloading the main body of the paper. Many of the technical details on deriving Fokker-Planck equations can also be found in Ref. 14. Time derivatives will be written as $\frac{\partial}{\partial t}$ though perhaps $\frac{d}{d t}$ should sometimes be used to emphasise that all time dependences are differentiated. 


\section{Theory - Separate Modes Treatment}

Phase space theory is based on the idea of representing the quantum density operator $\hat{\rho}$ by a quasi-distribution function $P\left(\alpha, \alpha^{+}, \alpha^{*}, \alpha^{+*}\right)$ which depends on phase variables $\alpha=\left\{\alpha_{1}, \alpha_{2}, . ., \alpha_{k}, . ., \alpha_{n}\right\}, \alpha^{+}=\left\{\alpha_{1}^{+}, \alpha_{2}^{+}, . ., \alpha_{k}^{+}, . ., \alpha_{n}^{+}\right\}$. Here $\alpha_{k}$ is associated with the annihilation operator $\widehat{a}_{k}$ and $\alpha_{k}^{+}$is associated with the creation operator $\widehat{a}_{k}^{\dagger}$ for the $k$ th mode involved in an expansion of the field operators $\hat{\Psi}(\mathbf{r})$ and $\hat{\Psi}^{\dagger}(\mathbf{r})$ in terms of orthonormal mode functions $\phi_{k}(\mathbf{r}, t)$ or their complex conjugates $\phi_{k}^{*}(\mathbf{r}, t)$. Quantum correlation functions can be expressed in terms of phase space averages involving the quasi-distribution function. The time dependence of the distribution function is given by a Fokker-Planck equation which includes drift and diffusion terms associated with first and second order derivatives of the distribution function with respect to the phase variables. Ultimately the phase space variables will be replaced by stochastic variables satisfying Ito stochastic equations that are equivalent to the Fokker-Planck equation, and the quantum correlation determined via stochastic averages. In the present paper dealing with time dependent mode functions, a hybrid distribution function is used where the condensate modes are treated via a Wigner distribution function and the non-condensate modes via a positive $P$ distribution function. Thus the phase variables for the condensate modes will be treated differently to those for the non-condensate modes. The treatment involves using time dependent phase variables, and leads to non-standard Fokker-Planck and Ito equations. Details of derivations are covered in Appendix 8 ,

\subsection{Field Operators, Hamiltonians, Quantum Correlation Functions}

In the Schrodinger picture the field operators associated with annihilating or creating a boson at position $\mathbf{r}$ are time independent and satisfy the standard bosonic commutation rules, for which the non-zero expressions are

$$
\left[\hat{\Psi}(\mathbf{r}), \hat{\Psi}^{\dagger}(\mathbf{s})\right]=\delta(\mathbf{r}-\mathbf{s})
$$

For simplicity we consider only single component bosonic systems, however cases where the bosons may involve differing internal (hyperfine) states can be treated via appropriate generalisations of the present approach.

The field operators are fundamental, appearing in expressions for the system Hamiltonian

$$
\hat{H}=\int d \mathbf{r}\left(\frac{\hbar^{2}}{2 m} \nabla \hat{\Psi}(\mathbf{r})^{\dagger} \cdot \nabla \hat{\Psi}(\mathbf{r})+\hat{\Psi}(\mathbf{r})^{\dagger} V \hat{\Psi}(\mathbf{r})+\frac{g}{2} \hat{\Psi}(\mathbf{r})^{\dagger} \hat{\Psi}(\mathbf{r})^{\dagger} \hat{\Psi}(\mathbf{r}) \hat{\Psi}(\mathbf{r})\right)
$$

where $m$ is the mass, $V$ is the trapping potential and the zero range approximation is used for interactions between the particles, with $g=4 \pi \hbar^{2} a_{s} / m$ involving the s-wave scattering length $a_{s}$.

In addition, measurement results are often expressed in terms of quantum correlation functions, which involve expectation values of products of field op- 
erators. A typical quantum correlation function is the normally ordered case

$$
\begin{aligned}
& G^{n}\left(\mathbf{r}_{1} \cdots \mathbf{r}_{p} ; \mathbf{s}_{q} \cdots \mathbf{s}_{1}\right)=\left\langle\hat{\Psi}\left(\mathbf{r}_{1}\right)^{\dagger} \cdots \hat{\Psi}\left(\mathbf{r}_{p}\right)^{\dagger} \hat{\Psi}\left(\mathbf{s}_{q}\right) \cdots \hat{\Psi}\left(\mathbf{s}_{1}\right)\right\rangle \\
= & \operatorname{Tr}\left(\hat{\Psi}\left(\mathbf{s}_{q}\right) \cdots \hat{\Psi}\left(\mathbf{s}_{1}\right) \hat{\rho}(t) \hat{\Psi}\left(\mathbf{r}_{1}\right)^{\dagger} \cdots \hat{\Psi}\left(\mathbf{r}_{p}\right)^{\dagger}\right)
\end{aligned}
$$

where for an $N$ particle system we require $p, q \leq N$ to give a non-zero result. Various spatial interference and coherence effects in Bose Einstein condensates can be described via such quantum correlation functions. The case for $p=q$ where $\mathbf{r}_{i}=\mathbf{s}_{i}$ for all $i$ is proportional to the probability of simultaneously detecting bosons at $\mathbf{r}_{1}, \mathbf{r}_{2} \cdots \mathbf{r}_{p}$ [41, 39].

\subsection{Time Dependent Modes}

For reasons set out in the Introduction, the phase space theory presented here is based on using time dependent mode functions to describe single particle states of the bosonic system. Such mode functions are required to be orthonormal and to form a complete set in the function space of interest. Thus the mode functions $\phi_{k}(x, t)$ satisfy

$$
\begin{aligned}
\int d x \phi_{k}^{*}(x, t) \phi_{l}(x, t) & =\delta_{k l} \\
\sum_{k} \phi_{k}(x, t) \phi_{k}^{*}(y, t) & =\delta(x-y)
\end{aligned}
$$

Henceforth $x$ refers to position in a 3D space unless otherwise stated.

For the case where the set of modes is not complete and restricted to a set $S$ the completeness relation is replaced by

$$
\sum_{k \in S} \phi_{k}(x, t) \phi_{k}^{*}(y, t)=\delta^{S}(x, y)
$$

where $\delta^{S}(x, y)$ is a restricted delta function [26, [14. The restricted delta function can be used to define a projector $\mathcal{P}_{x}^{S}$ that turns any function $F(y)$ into a linear combination of the $\phi_{k}(x, t)$ within the restricted function space. Thus

$$
\begin{aligned}
\mathcal{P}_{x}^{S}[F(x)] & =\int d y \delta^{S}(x, y) F(y) \\
& =\sum_{k \epsilon S} \phi_{k}(x, t) \int d y \phi_{k}^{*}(y, t) F(y)
\end{aligned}
$$

If the set of modes is complete then $\delta^{S}(x, y)=\delta(x-y)$, the usual Dirac delta function. Unless otherwise stated we will consider sets of modes $\phi_{k}(x, t)$ or $\phi_{k}^{*}(x, t)$ that are complete. 


\subsection{Mode Creation and Annihilation Operators}

The field operators may be expanded in terms of a complete set of time dependent orthonormal single mode functions as

$$
\hat{\Psi}(x)=\sum_{k} \widehat{a}_{k}(t) \phi_{k}(x, t), \quad \hat{\Psi}^{\dagger}(x)=\sum_{k} \widehat{a}_{k}^{\dagger}(t) \phi_{k}^{*}(x, t)
$$

where $\widehat{a}_{k}(t), \widehat{a}_{k}^{\dagger}(t)$ are time dependent mode annihilation, creation operators. These operators satisfy standard bosonic commutation rules that are equivalent to (1) for which the non-zero expressions are

$$
\left[\widehat{a}_{k}(t), \widehat{a}_{l}^{\dagger}(t)\right]=\delta_{k l}
$$

The annihilation, creation operators can be expressed in terms of the field operators and mode functions as

$$
\widehat{a}_{k}(t)=\int d x \phi_{k}^{*}(x, t) \hat{\Psi}(x) \quad \widehat{a}_{k}^{\dagger}(t)=\int d x \phi_{k}(x, t) \hat{\Psi}^{\dagger}(x)
$$

so they can be considered as functionals of the mode function or its complex conjugate.

A necessary and sufficient condition for the total field operators to be time independent is that the mode annihilation, creation operators evolve as

$$
\begin{aligned}
\frac{\partial \widehat{a}_{k}(t)}{\partial t} & =\sum_{l} C_{k l}(t) \widehat{a}_{l}(t) \quad \frac{\partial \widehat{a}_{k}^{\dagger}(t)}{\partial t}=\sum_{l} C_{k l}^{*}(t) \widehat{a}_{l}^{\dagger}(t) \\
C_{k l}(t) & =\int d x \frac{\partial \phi_{k}^{*}(x, t)}{\partial t} \phi_{l}(x, t)=i D_{k l}(t)
\end{aligned}
$$

showing that the time derivatives of the annihilation, creation operators are just linear combinations of these operators. Note that the sum is over all modes. By differentiating the orthonormality condition (4) we see that

$$
C_{k l}+C_{l k}^{*}=0
$$

so it follows that the related matrix $D$ is hermitian, $D_{k l}=D_{l k}^{*}$. We will refer to the $C_{k l}(t)$ as the coupling coefficients. As will be seen later the coupling coefficients play a key role in the functional Fokker-Planck and Ito stochastic field equations.

Assuming that the mode functions $\phi_{k}(x, t)$ or $\phi_{k}^{*}(x, t)$ form a complete set for expansions of $\frac{\partial}{\partial t} \phi_{k}(x, t)$ or $\frac{\partial}{\partial t} \phi_{k}^{*}(x, t)$ respectively, we can also expand the mode derivatives in terms of the modes with the coupling coefficients as expansion coefficients

$$
\begin{aligned}
\frac{\partial}{\partial t} \phi_{k}(x, t) & =\sum_{l} \beta_{k l} \phi_{l}(x, t)=\sum_{l} C_{k l}^{*} \phi_{l}(x, t) \\
\frac{\partial}{\partial t} \phi_{k}^{*}(x, t) & =\sum_{l} C_{k l} \phi_{l}^{*}(x, t)
\end{aligned}
$$


We can use these results to confirm that the mode expansions for the field operators are time independent. We have using (8), (11) and (14)

$$
\begin{aligned}
\frac{\partial}{\partial t} \widehat{\psi}(x) & =0 \\
\frac{\partial}{\partial t} \widehat{\psi}^{\dagger}(x) & =0
\end{aligned}
$$

after applying (13).

\subsection{Determination of Mode Functions}

There are several possibilities for determining the time dependent modes. One possibility is to use the complex conjugates of the eigenfunctions for the first order quantum correlation function $G^{1}\left(\mathbf{r}_{1} ; \mathbf{s}_{1}\right)=\left\langle\hat{\Psi}\left(\mathbf{r}_{1}\right)^{\dagger} \hat{\Psi}\left(\mathbf{s}_{1}\right)\right\rangle$ - these are the so-called natural orbitals. The corresponding eigenvalues give the occupancies of these single boson states, and the condensate modes could be chosen as those with macroscopic occupancy, with the remainder specifying non-condensate modes. However this requires knowing the quantum correlation function, and it is hard to see how this could be done without first determining the dynamical behaviour. Another possibility would be to use the time dependent single particle states for a single boson in the trap potential, but this ignores the effect of boson-boson interactions. Perhaps the best alternative is to apply the Dirac-Frenkel variational principle [30, 31] to a state in which the bosons are restricted to only occupying as few modes as possible, and where the mode functions along with amplitudes for the allowed Fock states are treated as variational functions. Such an approach leads to the Gross-Pitaevskii equation [28], [29] in the case where only one mode is involved or generalised Gross-Pitaevskii equations where two or more modes are considered.

The Dirac-Frenkel principle involves minimising the dynamical action given by

$$
S_{d y n}=\int d t\left(\begin{array}{c}
\left\{\left\langle\partial_{t} \Phi \mid \Phi\right\rangle-\left\langle\Phi \mid \partial_{t} \Phi\right\rangle\right\} / 2 i \\
-\langle\Phi|\widehat{H}| \Phi\rangle / \hbar
\end{array}\right)
$$

where $|\Phi(t)\rangle$ is the quantum state. For unrestricted states this minimisation leads to the time-dependent Schrodinger equation, so the Dirac-Frenkel principle enables the fundamental equation for quantum dynamics to be expressed as a Principle of Least Action. In the present application however, the quantum state is restricted to a specific form involving variational quantities, so applying the Dirac-Frenkel principle then does not result in a quantum state that satisfies the time-dependent Schrodnger equation, but nevertheless is as close to being a solution as that the specific form allows.

For the two mode case that is relevant to double well BEC interferometry the two condensate mode functions are determined as in [14, [39. The quantum state $|\Phi(t)\rangle$ of the $N$ boson system as a superposition of the $N+1$ basis states $\left|\frac{N}{2}, k\right\rangle$, where there are $\frac{N}{2}-k$ and $\frac{N}{2}+k$ bosons (respectively) occupying the 
two modes with (time dependent) mode functions $\phi_{1}(x, t)$ and $\phi_{2}(x, t)$. The amplitude for this basis state is $b_{k}(t)$.

$$
|\Phi(t)\rangle=\sum_{k=-\frac{\mathbf{N}}{2}}^{\frac{\mathbf{N}}{2}} b_{k}(t)\left|\frac{N}{2}, k\right\rangle
$$

and the basis states are Fock states given by

$$
\left|\frac{N}{2}, k\right\rangle=\frac{\left(\widehat{a}_{1}(t)^{\dagger}\right)^{\left(\frac{N}{2}-k\right)}}{\left[\left(\frac{N}{2}-k\right) !\right]^{\frac{1}{2}}} \frac{\left(\widehat{a}_{2}(t)^{\dagger}\right)^{\left(\frac{N}{2}+k\right)}}{\left[\left(\frac{N}{2}+k\right) !\right]^{\frac{1}{2}}}|0\rangle \quad(k=-N / 2,-N / 2+1, . .,+N / 2)
$$

These basis states are fragmented or number squeezed states, allowing for both modes to have macroscopic occupancy when $|k| \ll N / 2$. The notation $\left|\frac{N}{2}, k\right\rangle$ for the basis states reflects the feature that the two mode Bose condensate behaves like a giant spin system - details are given in [14], [39]. The total angular momentum quantum number $j=\frac{N}{2}$ is macroscopic, and $k=-\frac{N}{2},-\frac{N}{2}+1, . ., \frac{N}{2}-1,+\frac{N}{2}$ specifies the magnetic quantum number as well as $2 k$ determining the difference in mode occupancy.

The quantum state (17) is a functional of the amplitudes $b_{k}$ and (via (10)) the mode functions $\phi_{1}(x, t)$ and $\phi_{2}(x, t)$. In [14], 39] a self-consistent set of equations for the amplitudes and mode functions has been determined by applying the Dirac-Frenkel principle 30, 31 and minimising the dynamic action with respect to these variational functions using the state vector given by (17). Here the mode functions and amplitudes act as variational quantities.

The mode functions satisfy the coupled generalised Gross-Pitaevskii equations

$$
\begin{aligned}
i \hbar \sum_{j} X_{i j} \frac{\partial}{\partial t} \phi_{j}= & \sum_{j} X_{i j}\left(-\frac{\hbar^{2}}{2 m} \nabla^{2}+V\right) \phi_{j} \\
& +\sum_{j}\left(g \sum_{m n} Y_{i m j n} \phi_{m}^{*} \phi_{n}\right) \phi_{j} \quad(i=1,2) .
\end{aligned}
$$

and allow for boson-boson interactions and are time-dependent. They follow the changes in the time dependent potential $V(\mathbf{r}, t)$. This is a generalised mean field theory - the quantity in brackets in the final term of the last equation being a mean field. The quantities $X_{i j}$ and $Y_{i m}$ jn are one-body and two-body correlation functions

$$
\begin{aligned}
X_{i j} & =\left\langle\Phi\left|\widehat{a}_{i}^{\dagger} \widehat{a}_{j}\right| \Phi\right\rangle \\
Y_{i m j n} & =\left\langle\Phi\left|\widehat{a}_{i}^{\dagger} \widehat{a}_{m}^{\dagger} \widehat{a}_{j} \widehat{a}_{n}\right| \Phi\right\rangle
\end{aligned}
$$

Detailed expressions given in the Appendix for [14], 39], showing that $X_{i j}$ and $Y_{i m j n}$ are quadratic forms of the amplitudes $b_{k}$. They are of order $N$ and $N^{2}$ respectively.

The amplitudes satisfy coupled matrix mechanics equations

$$
i \hbar \frac{\partial b_{k}}{\partial t}=\sum_{l}\left(H_{k l}-\hbar U_{k l}\right) b_{l} \quad(k=-N / 2, . ., N / 2) .
$$


These $N+1$ equations (22) describe the system dynamics as it evolves amongst the possible fragmented states. In these equations the matrix elements $H_{k l}$, $U_{k l}$ depend on the mode functions $\phi_{i}(x, t)$. Detailed expressions for $H_{k l}, U_{k l}$ are given in Appendix for 14, 39. The quantities $H_{k l}$ are matrix elements of the Hamiltonian $\widehat{H}$ in equation (2) between the fragmented states $\left|\frac{N}{2}, k\right\rangle$, $\left|\frac{N}{2}, l\right\rangle$. The quantities $U_{k l}$ are elements of the so-called rotation matrix, and allow for the time dependence of the mode functions.

$$
\begin{aligned}
H_{k l} & =\left\langle\frac{N}{2}, k|\widehat{H}| \frac{N}{2}, l\right\rangle \\
U_{k l} & =\frac{1}{2 i}\left(\left\{\partial_{t}\left\langle\frac{N}{2}, k\right|\right\}\left|\frac{N}{2}, l\right\rangle-\left\langle\frac{N}{2}, k\right|\left\{\partial_{t}\left|\frac{N}{2}, l\right\rangle\right\}\right)
\end{aligned}
$$

The specific forms of the $X_{i j}, Y_{i m j n}, H_{k l}, U_{k l}$ are not important in what follows, all that is required is that they have been determined. Equations for the mode functions and amplitudes similar to (19) and (22) have been obtained by Alon et al [37. for single component BECs. The key feature is the self-consistent nature of the equations - the amplitudes are required to determine the form of the mode equations and the mode functions are required to determine the matrix elements in the amplitude equations.

From the amplitude and mode equations it can be shown that

$$
\begin{aligned}
\frac{\partial}{\partial t} \sum_{k=-\frac{\mathrm{N}}{2}}^{\frac{\mathrm{N}}{2}}\left|b_{k}(t)\right|^{2} & =0 \\
i \hbar \sum_{i j} X_{i j} \frac{\partial}{\partial t} \int d x \phi_{i}^{*}(x, t) \phi_{j}(x, t) & =0
\end{aligned}
$$

The first result shows that the amplitudes would remain normalised to unity and the second result is consistent with the modes remaining orthogonal and normalised, assuming they were so chosen at $t=0$. The second result involves the trace of the product of a positive definite invertible matrix $X$ with a matrix which is the time derivative of the mode orthogonality matrix.

For the non-condensate modes a different approach is required since physical considerations suggest the mode occupancy would be small. The variational approach might be extended to consider quantum states for an $N+1$ boson system where all except a single boson occupy one or two condensate modes. For the case of two condensate modes such a quantum state would be of the form

$$
|\Phi(t)\rangle=\sum_{k=-\frac{\mathrm{N}}{2}}^{\frac{\mathrm{N}}{2}} \sum_{l} b_{k, l}(t) \frac{\left(\widehat{a}_{1}(t)^{\dagger}\right)^{\left(\frac{N}{2}-k\right)}}{\left[\left(\frac{N}{2}-k\right) !\right]^{\frac{1}{2}}} \frac{\left(\widehat{a}_{2}(t)^{\dagger}\right)^{\left(\frac{N}{2}+k\right)}}{\left[\left(\frac{N}{2}+k\right) !\right]^{\frac{1}{2}}}\left(\widehat{a}_{l}(t)^{\dagger}\right)|0\rangle
$$

where the variational functions are now the two condensate mode functions $\phi_{1}(x, t), \phi_{2}(x, t)$, the non-condensate mode functions $\phi_{l}(x, t)$ and the state amplitudes $b_{k, l}(t)$. Presumably the mode equations for the condensate modes would 
be similar to those discussed above, whilst the mode equation for the singly occupied non-condensate modes would contain mean field terms associated with the highly occupied condensate modes - rather like what happens for the highest energy orbital for electrons in an alkali metal atom, the closed shell orbitals being analogous to the condensate modes. There would be a multitude of time dependent solutions for the singly occupied mode, and these could be used as the non-condensate mode functions. Alternatively, the non-condensate modes might be just chosen via a Schmidt orthogonality process to be orthogonal to the previous condensate modes, starting with a set of plane wave modes or single atom trapped modes.

\subsection{Characteristic and Distribution Functions}

Phase space theory involves first introducing characteristic functions that can be used to specify all the quantum correlation functions for a given density operator. We wish to divide the modes up into two sets - condensate and noncondensate modes. The former are to be treated via the Wigner distribution, the latter via the positive $\mathrm{P}$ distribution. The definition of the characteristic function $\chi\left(\xi, \xi^{+}\right)$for this hybrid approach is

$$
\begin{aligned}
\chi\left(\xi, \xi^{+}\right) & =\operatorname{Tr}\left(\hat{\Omega}_{C}^{W}\left(\xi_{C}, \xi_{C}^{+}\right) \hat{\Omega}_{N C}^{+}\left(\xi_{N C}^{+}\right) \hat{\rho} \hat{\Omega}_{N C}^{-}\left(\xi_{N C}\right)\right) \\
\hat{\Omega}_{N C}^{+}\left(\xi_{N C}^{+}\right) & =\exp i \sum_{k \in N C} \hat{a}_{k} \xi_{k}^{+} \quad \hat{\Omega}_{N C}^{-}\left(\xi_{N C}\right)=\exp i \sum_{k \in N C} \xi_{k} \hat{a}_{k}^{\dagger} \\
\hat{\Omega}_{C}^{W}\left(\xi_{C}, \xi_{C}^{+}\right) & =\exp i \sum_{k \in C}\left(\hat{a}_{k} \xi_{k}^{+}+\xi_{k} \hat{a}_{k}^{\dagger}\right)
\end{aligned}
$$

where the characteristic variables are $\xi=\left\{\xi_{1}, \xi_{2}, . ., \xi_{k}, . . \xi_{n}\right\}=\left\{\xi_{C}, \xi_{N C}\right\}, \xi^{+}=$ $\left\{\xi_{1}^{+}, \xi_{2}^{+}, . ., \xi_{k}^{+}, . . \xi_{n}^{+}\right\}=\left\{\xi_{C}^{+}, \xi_{N C}^{+}\right\}$. There are two sub-sets - condensate $\xi_{C}$ or $\xi_{C}^{+}$and non-condensate $\xi_{N C}$ or $\xi_{N C}^{+}$

Using the Baker-Hausdorff theorem

$\exp i \sum_{k}\left(\hat{a}_{k} \xi_{k}^{+}+\xi_{k} \hat{a}_{k}^{\dagger}\right)=\left(\exp i \sum_{k} \xi_{k} \hat{a}_{k}^{\dagger}\right)\left(\exp i \sum_{k} \hat{a}_{k} \xi_{k}^{+}\right)\left(\exp \left(-\frac{1}{2} \sum_{k} \xi_{k} \xi_{k}^{+}\right)\right)$

and the cyclic properties of the trace, we may combine exponential operators to find that

$$
\begin{aligned}
\chi\left(\xi, \xi^{+}\right) & =\exp \left(-\frac{1}{2} \sum_{k \in C} \xi_{k} \xi_{k}^{+}\right) \chi_{P+}\left(\xi, \xi^{+}\right) \\
\chi_{P+}\left(\xi, \xi^{+}\right) & =\operatorname{Tr}\left(\hat{\Omega}^{+}\left(\xi^{+}\right) \hat{\rho} \hat{\Omega}^{-}(\xi)\right) \\
\hat{\Omega}^{+}\left(\xi^{+}\right) & =\exp i \sum_{k \in C, N C} \hat{a}_{k} \xi_{k}^{+} \quad \hat{\Omega}^{-}(\xi)=\exp i \sum_{k \in C, N C} \xi_{k} \hat{a}_{k}^{\dagger}
\end{aligned}
$$

which relates the hybrid characteristic function to that for the case where all modes are treated via a normally ordered characteristic function. 
The characteristic function is related to the quasi-distribution function $P\left(\alpha, \alpha^{+}, \alpha^{*}, \alpha^{+*}\right)$ via

$$
\begin{aligned}
& \chi\left(\xi, \xi^{+}\right) \\
= & \iint d^{2} \alpha d^{2} \alpha^{+} \\
& \times \exp \left(i \sum_{k \epsilon C}\left(\alpha_{k} \xi_{k}^{+}+\xi_{k} \alpha_{k}^{+}\right)\right) \exp \left(i \sum_{k \in N C} \alpha_{k} \xi_{k}^{+}\right) P\left(\alpha, \alpha^{+}, \alpha^{*}, \alpha^{+*}\right) \exp \left(i \sum_{k \in N C} \xi_{k} \alpha_{k}^{+}\right) \\
= & \iint d^{2} \alpha d^{2} \alpha^{+} \exp \left(i \sum_{k \epsilon C, N C}\left(\alpha_{k} \xi_{k}^{+}+\xi_{k} \alpha_{k}^{+}\right)\right) P\left(\alpha, \alpha^{+}, \alpha^{*}, \alpha^{+*}\right)
\end{aligned}
$$

where in the second line all the exponentials have been combined. As in [14, 25], the hybrid quasi-distribution function is of the Wigner type for the condensate modes and of the positive $\mathrm{P}$ type for the non-condensate modes. The phase space variables are $\alpha=\left\{\alpha_{1}, \alpha_{2}, . ., \alpha_{k}, . ., \alpha_{n}\right\}=\left\{\alpha_{C}, \alpha_{N C}\right\}, \alpha^{+}=$ $\left\{\alpha_{1}^{+}, \alpha_{2}^{+}, . ., \alpha_{k}^{+}, . ., \alpha_{n}^{+}\right\}=\left\{\alpha_{C}^{+}, \alpha_{N C}^{+}\right\}$, where the set of phase space variables for the condensate modes is $\alpha_{C}$ or $\alpha_{C}^{+}$and that for the non-condensate modes is $\alpha_{N C}$ or $\alpha_{N C}^{+}$. With $\alpha_{k}=\alpha_{k x}+i \alpha_{k y}, \alpha_{k}^{+}=\alpha_{k x}^{+}+i \alpha_{k y}^{+}$the phase space integration is

$$
\iint d^{2} \alpha d^{2} \alpha^{+} \equiv \iint \prod_{k} d \alpha_{k x} d \alpha_{k y} \prod_{k} d \alpha_{k x}^{+} d \alpha_{k y}^{+}
$$

It needs to be emphasised for this double phase space approach that although the characteristic function is uniquely determined from the density operator and vice versa, the same is not true for the distribution function. As emphasised in several references 42, 443 dealing with the positive $\mathrm{P}$ representation, the distribution is non-unique - many equivalent distribution functions determine the same characteristic function and density operator, and these may satisfy different Fokker-Planck equations based on different choices of the correspondence rules used to convert the dynamical equation for the density operator into an equivalent Fokker-Planck equation for the distribution function (see Section 2.8). Although this non-uniqueness precludes interpreting the distribution function as a probability, it is seen as having the advantage of allowing flexibility in the Fokker-Planck equations in terms of obtaining related Ito stochastic equations that are suitable for numerical work.

Although the distribution function is not unique, it is known to exist - at least for the positive $\mathrm{P}$ case. In that case 42 proved the existence of a so-called canonical form of the positive $\mathrm{P}$ distribution function in the form

$$
\begin{aligned}
& P_{\text {canon }}\left(\alpha, \alpha^{+}, \alpha^{*}, \alpha^{+*}\right) \\
= & \left(\frac{1}{4 \pi^{2}}\right)^{n} \exp \left(-\frac{1}{2} \sum_{k}\left(\alpha_{k} \alpha_{k}^{*}+\alpha_{k}^{+*} \alpha_{k}^{+}\right)\right) \times\left\langle\left.\frac{\alpha+\alpha^{+*}}{2}\right|_{B} \widehat{\rho} \mid \frac{\alpha+\alpha^{+*}}{2}\right\rangle_{B}
\end{aligned}
$$

where

$$
|\alpha\rangle_{B}=\exp \left(\sum_{k} \alpha_{k} \widehat{a}_{k}^{\dagger}\right)|0\rangle
$$


are Bargmann coherent states and where the density operator may be written as

$$
\hat{\rho}=\iint d^{2} \alpha^{+} d^{2} \alpha P_{\text {canon }}\left(\alpha, \alpha^{+}, \alpha^{*}, \alpha^{+*}\right) \frac{|\alpha\rangle_{B}\left\langle\left.\alpha^{+*}\right|_{B}\right.}{\operatorname{Tr}\left(|\alpha\rangle_{B}\left\langle\left.\alpha^{+*}\right|_{B}\right)\right.}
$$

\subsection{Quantum Correlation Functions}

The standard approach can be applied to relate the quantum correlation functions to derivatives of the characteristic functions with respect to $i \xi_{k}$ or $i \xi_{k}^{+}$followed by letting the $\xi_{k}, \xi_{k}^{+} \rightarrow 0$. This enables the quantum correlation functions to be expressed as phase space integrals. The quantum correlation functions are for symmetrically ordered products of condensate mode operators and normally ordered products for non-condensate mode operators. We have

$$
\begin{aligned}
& G\left(l_{1}, l_{2}, . . l_{p} ; m_{q}, . ., m_{2}, m_{1} \quad: \quad j_{1}, j_{2}, . . j_{r} ; k_{s}, . ., k_{2}, k_{1}\right) \\
& =\left\langle\left\{\hat{a}_{l_{1}}^{\dagger} \hat{a}_{l_{2}}^{\dagger} . . \hat{a}_{l_{n}}^{\dagger} \hat{a}_{m_{q}} . \hat{a}_{m_{2}} \hat{a}_{m_{1}}\right\} \hat{a}_{j_{1}}^{\dagger} \hat{a}_{j_{2}}^{\dagger} . . \hat{a}_{j_{r}}^{\dagger} \hat{a}_{k_{s}} . . \hat{a}_{k_{2}} \hat{a}_{k_{1}}\right\rangle \\
& =\operatorname{Tr}\left(\hat{\rho}\left\{\hat{a}_{l_{1}}^{\dagger} \hat{a}_{l_{2}}^{\dagger} . . \hat{a}_{l_{p}}^{\dagger} \hat{a}_{m_{q}} . . \hat{a}_{m_{2}} \hat{a}_{m_{1}}\right\} \hat{a}_{j_{1}}^{\dagger} \hat{a}_{j_{2}}^{\dagger} . . \hat{a}_{j_{r}}^{\dagger} \hat{a}_{k_{s}} . . \hat{a}_{k_{2}} \hat{a}_{k_{1}}\right) \\
& =\iint d^{2} \alpha d^{2} \alpha^{+} P\left(\alpha, \alpha^{+}, \alpha^{*}, \alpha^{+*}\right) \\
& \times\left[\alpha_{l_{1}}^{+} \alpha_{l_{2}}^{+} . . \alpha_{l_{p}}^{+} \alpha_{m_{q}} . . \alpha_{m_{2}} \alpha_{m_{1}}\right]\left[\alpha_{j_{1}}^{+} \alpha_{j_{2}}^{+} . . \alpha_{j_{r}}^{+} \alpha_{k_{s}} . . \alpha_{k_{2}} \alpha_{k_{1}}\right]
\end{aligned}
$$

where $l_{1}, l_{2}, . . l_{p} ; m_{q}, . ., m_{2}, m_{1}$ refer to condensate modes and $j_{1}, j_{2}, . . j_{r} ; k_{s}, . ., k_{2}, k_{1}$ to non-condensate modes. Symmetrically ordered means the average of the product of the operators taken in any order. Thus

$$
\begin{aligned}
& \left\{\hat{a}_{l_{1}}^{\dagger} \hat{a}_{l_{2}}^{\dagger} . . \hat{a}_{l_{p}}^{\dagger} \hat{a}_{m_{q}} . . \hat{a}_{m_{2}} \hat{a}_{m_{1}}\right\} \\
= & \frac{1}{(p+q) !} \sum_{R} \Re\left(\hat{a}_{l_{1}}^{\dagger} \hat{a}_{l_{2}}^{\dagger} . . \hat{a}_{l_{p}}^{\dagger} \hat{a}_{m_{q}} . . \hat{a}_{m_{2}} \hat{a}_{m_{1}}\right) .
\end{aligned}
$$

In Eq.(36) the sum over $R$ is over all $(p+q)$ ! orderings $\Re$ of the factors $\hat{a}_{l_{1}}^{\dagger} \hat{a}_{l_{2}}^{\dagger} . . \hat{a}_{l_{p}}^{\dagger} \hat{a}_{m_{q}} . . \hat{a}_{m_{2}} \hat{a}_{m_{1}}$.

\subsection{Time Dependent Phase Variables}

As outlined in the Introduction, the complex phase variables $\alpha_{k}(t), \alpha_{k}^{+}(t)$ associated with the time dependent mode annihilation, creation operators $\widehat{a}_{k}(t), \widehat{a}_{k}^{\dagger}(t)$ will be chosen to be time dependent. Hybrid quasi-distribution functions $P\left(\alpha, \alpha^{+}, \alpha^{*}, \alpha^{+*}\right)$ in double phase spaces will be considered in which the condensate modes will be treated in terms of Wigner and non-condensate modes in terms of positive $\mathrm{P}$ quasi-distribution functions. Note that the distribution function $P\left(\alpha, \alpha^{+}, \alpha^{*}, \alpha^{+*}\right)$ is not an analytic function of the $\alpha_{k}(t), \alpha_{k}^{+}(t)$ but also depends on the complex conjugates $\alpha_{k}^{*}(t), \alpha_{k}^{+*}(t)$. The time dependence of the phase variables is arbitrary, but since they are associated with the mode annihilation, creation operators one logical choice would be to require the phase variables to have the same 
time dependence. As we will see, this leads to theoretical results that allow for the consequences of the basic time dependence of the mode functions is a reasonably simple form. In particular, the field functions that are associated with the time independent total field operators are also time independent, as might be hoped for. An alternative choice of conventional time independent phase variables would lead to an unsatisfactory result that time dependent field functions are associated with the total field operators. The theory can of course also be developed via such other choices of time dependence for the phase variables, we believe however that the present choice is the most satisfactory.

The time dependences chosen for the phase variables are thus as for the mode annihilation, creation operators

$$
\frac{\partial \alpha_{k}(t)}{\partial t}=\sum_{l} C_{k l}(t) \alpha_{l}(t) \quad \frac{\partial \alpha_{k}^{+}(t)}{\partial t}=\sum_{l} C_{k l}^{*}(t) \alpha_{l}^{+}(t)
$$

and since $C_{k l}(t)=i D_{k l}(t)$ with $D$ hermitian, it is possible to find a unitary matrix $T$ given by

$$
\frac{\partial T_{k l}(t)}{\partial t}=i \sum_{m} D_{k m}(t) T_{m l}(t)
$$

such that the formal solution for the phase variables is

$$
\alpha_{k}(t)=\sum_{l} T_{k l}(t) \alpha_{l}(0) \quad \alpha_{k}^{+}(t)=\sum_{l} T_{k l}^{*}(t) \alpha_{l}^{+}(0)
$$

This choice of the time dependence for the phase variables is a key element in the derivations.

By writing $\alpha_{k}(t)=\alpha_{k x}(t)+i \alpha_{k y}(t), \alpha_{k}^{+}(t)=\alpha_{k x}^{+}(t)+i \alpha_{k y}^{+}(t), T_{k l}(t)=$ $T_{k l}^{x}(t)+i T_{k l}^{y}(t)$ in terms of real, imaginary parts, it is then possible to relate phase space integrals over the $\alpha(t), \alpha^{+}(t)$ to phase space integrals over the $\alpha(0), \alpha^{+}(0)$. Thus

$$
\begin{aligned}
\iint d^{2} \alpha(t) d^{2} \alpha^{+}(t) & \equiv \iint \prod_{k} d \alpha_{k x}(t) d \alpha_{k y}(t) \prod_{k} d \alpha_{k x}^{+}(t) d \alpha_{k y}^{+}(t) \\
& =\iint \prod_{k} d \alpha_{k x}(0) d \alpha_{k y}(0) \prod_{k} d \alpha_{k x}^{+}(0) d \alpha_{k y}^{+}(0) \\
& =\iint d^{2} \alpha(0) d^{2} \alpha^{+}(0)
\end{aligned}
$$

The Jacobian involves the $T_{k l}(t), T_{k l}^{*}(t)$ and the unitarity of $T$ leads to the Jacobian being unity. Differentiation under the phase space integral can therefore be carried out without concern about time dependent differentials - a key simplification for the derivations.

The time dependence of the characteristic function variables $\xi=\left\{\xi_{1}, \xi_{2}, . ., \xi_{k}, . . \xi_{n}\right\}, \xi^{+}=$ $\left\{\xi_{1}^{+}, \xi_{2}^{+}, . ., \xi_{k}^{+}, . . \xi_{n}^{+}\right\}$is also arbitrary. However, if we choose the $\xi_{k}(t), \xi_{k}^{+}(t)$ to evolve as for the mode annihilation, creation operators

$$
\frac{\partial \xi_{k}(t)}{\partial t}=\sum_{l} C_{k l}(t) \xi_{l}(t) \quad \frac{\partial \xi_{k}^{+}(t)}{\partial t}=\sum_{l} C_{k l}^{*}(t) \xi_{l}^{+}(t)
$$


- which is the same as for the phase space variables, then this leads to many key expressions involving the characteristic variables being time independent, thereby simplifying the theory.

\subsection{Fokker-Planck Equations}

The Fokker-Planck equation gives the time dependence of the distribution function $P\left(\alpha, \alpha^{+}, \alpha^{*}, \alpha^{+*}\right)$. The derivation of it here requires special treatment due to the time dependent modes and phase space variables used in the theory.

\subsubsection{Derivation}

We first need to consider the time derivative of the characteristic function (28) in the form given by Eq. (30) It follows using Eqs.(11), (41) together with $C_{k l}+C_{l k}^{*}=0$ that

$$
\begin{aligned}
& \frac{\partial}{\partial t} \sum_{k} \hat{a}_{k} \xi_{k}^{+}=0 \\
& \frac{\partial}{\partial t} \sum_{k} \xi_{k} \hat{a}_{k}^{+}=0
\end{aligned}
$$

This means that the time derivative of the normally ordered characteristic function $\chi_{P+}\left(\xi, \xi^{+}\right)$is only dependent on the time derivative of the density operator, with the time dependences of the mode annihilation, creation operators $\widehat{a}_{k}(t), \widehat{a}_{k}^{\dagger}(t)$ and characteristic function variables $\xi_{k}(t), \xi_{k}^{+}(t)$ cancelling

out. However, although the quantities $\sum_{k \in C, N C} \hat{a}_{k} \xi_{k}^{+}$and $\sum_{k \in C, N C} \xi_{k} \hat{a}_{k}^{\dagger}$ are time independent, the same is not true for $\sum_{k \in C} \xi_{k} \xi_{k}^{+}$. We find that

$$
\frac{\partial}{\partial t} \sum_{k \in C} \xi_{k} \xi_{k}^{+}=\sum_{k \in C} \sum_{l \in N C}\left(C_{k l} \xi_{l} \xi_{k}^{+}+C_{k l}^{*} \xi_{k} \xi_{l}^{+}\right)
$$

where the $k \epsilon C, l \epsilon C$ terms cancel out. This means that there are additional terms in the equation of motion for the characteristic function. Thus

$$
\begin{aligned}
\frac{\partial}{\partial t} \chi\left(\xi, \xi^{+}\right)= & \left(-\frac{1}{2} \sum_{k \in C} \sum_{l \in N C}\left(C_{k l} \xi_{l} \xi_{k}^{+}+C_{k l}^{*} \xi_{k} \xi_{l}^{+}\right)\right) \exp \left(-\frac{1}{2} \sum_{k \in C} \xi_{k} \xi_{k}^{+}\right) \chi_{P+}\left(\xi, \xi^{+}\right) \\
& +\exp \left(-\frac{1}{2} \sum_{k \in C} \xi_{k} \xi_{k}^{+}\right) \operatorname{Tr}\left(\hat{\Omega}^{+}\left(\xi^{+}\right) \frac{\partial}{\partial t} \hat{\rho} \hat{\Omega}^{-}(\xi)\right) \\
= & \left(\frac{1}{2} \sum_{k \in C} \sum_{l \in N C}\left(C_{k l} i \xi_{l} i \xi_{k}^{+}+C_{k l}^{*} i \xi_{k} i \xi_{l}^{+}\right)\right) \chi\left(\xi, \xi^{+}\right)+\chi\left(\xi, \xi^{+} ; \frac{\partial}{\partial t} \hat{\rho}\right)
\end{aligned}
$$

The first term in (44) then produces extra terms in the Fokker-Planck equation. The last term in (44) is the characteristic function that would occur if $\hat{\rho}$ is 
replaced by $\frac{\partial}{\partial t} \hat{\rho}$ and this term just gives the standard result for the FokkerPlanck equation.

The first term on the right side of (44) is given by

$$
\begin{aligned}
& \left(\frac{1}{2} \sum_{k \in C} \sum_{l \epsilon N C}\left(C_{k l} i \xi_{l} i \xi_{k}^{+}+C_{k l}^{*} i \xi_{k} i \xi_{l}^{+}\right)\right) \chi\left(\xi, \xi^{+}\right) \\
= & \iint d^{2} \alpha d^{2} \alpha^{+} \exp \left(i \sum_{k \epsilon C, N C}\left(\alpha_{k} \xi_{k}^{+}+\xi_{k} \alpha_{k}^{+}\right)\right) \\
& \times\left\{\frac{1}{2} \sum_{k \in C} \sum_{l \epsilon N C}\left(C_{k l} \frac{\partial}{\partial \alpha_{l}^{+}} \frac{\partial}{\partial \alpha_{k}}+C_{k l}^{*} \frac{\partial}{\partial \alpha_{k}^{+}} \frac{\partial}{\partial \alpha_{l}}\right) P\left(\alpha, \alpha^{+}, \alpha^{*}, \alpha^{+*}\right)\right\}
\end{aligned}
$$

because using (31) and applying integration by parts twice we see that

$$
\begin{aligned}
& i \xi_{l} i \xi_{k}^{+} \chi\left(\xi, \xi^{+}\right) \\
= & \iint d^{2} \alpha d^{2} \alpha^{+}\left(\frac{\partial}{\partial \alpha_{l}^{+}} \frac{\partial}{\partial \alpha_{k}} \exp \left(i \sum_{k \epsilon C, N C}\left(\alpha_{k} \xi_{k}^{+}+\xi_{k} \alpha_{k}^{+}\right)\right)\right) P\left(\alpha, \alpha^{+}, \alpha^{*}, \alpha^{+*}\right) \\
= & \iint d^{2} \alpha d^{2} \alpha^{+} \exp \left(i \sum_{k \epsilon C, N C}\left(\alpha_{k} \xi_{k}^{+}+\xi_{k} \alpha_{k}^{+}\right)\right)\left\{\frac{\partial}{\partial \alpha_{l}^{+}} \frac{\partial}{\partial \alpha_{k}} P\left(\alpha, \alpha^{+}, \alpha^{*}, \alpha^{+*}\right)\right\} \\
= & i \xi_{k} i \xi_{l}^{+} \chi\left(\xi, \xi^{+}\right) \\
& \iint d^{2} \alpha d^{2} \alpha^{+} \exp \left(i \sum_{k \in C, N C}\left(\alpha_{k} \xi_{k}^{+}+\xi_{k} \alpha_{k}^{+}\right)\right)\left\{\frac{\partial}{\partial \alpha_{k}^{+}} \frac{\partial}{\partial \alpha_{l}} P\left(\alpha, \alpha^{+}, \alpha^{*}, \alpha^{+*}\right)\right\}
\end{aligned}
$$

The second term on the right side of (44) is evaluated using the correspondence rules that apply when $\frac{\partial}{\partial t} \hat{\rho}$ evaluated using the Liouville-von Neumann or master equation. The derivation of these rules is set out in many textbooks (see for example 44], Appendix 12) and here we just present the results. For the non-condensate annihilation, creation operators the characteristic function is of the positive $\mathrm{P}$ normally ordered type so we have

$$
\begin{array}{lll}
\hat{\rho} \Rightarrow \hat{a}_{k} \hat{\rho} & \chi\left(\xi, \xi^{+}\right) \Rightarrow \frac{\partial}{\partial\left(i \xi_{k}^{+}\right)} \chi \\
\hat{\rho} \Rightarrow \hat{\rho} \hat{a}_{k} & \chi\left(\xi, \xi^{+}\right) \Rightarrow\left(\frac{\partial}{\partial\left(i \xi_{k}^{+}\right)}+i \xi_{k}\right) \chi \\
\hat{\rho} \Rightarrow \hat{a}_{k}^{\dagger} \hat{\rho} & \chi\left(\xi, \xi^{+}\right) \Rightarrow\left(\frac{\partial}{\partial\left(i \xi_{k}\right)}+i \xi_{k}^{+}\right) \chi \\
\hat{\rho} \Rightarrow \hat{\rho} \hat{a}_{k}^{\dagger} & \chi\left(\xi, \xi^{+}\right) \Rightarrow \frac{\partial}{\partial\left(i \xi_{k}\right)} \chi
\end{array}
$$

For the condensate annihilation, creation operators the characteristic function 
is of the Wigner symmetrically ordered type so we have

$$
\begin{array}{lll}
\hat{\rho} \Rightarrow \hat{a}_{k} \hat{\rho} & \chi\left(\xi, \xi^{+}\right) \Rightarrow\left(\frac{\partial}{\partial\left(i \xi_{k}^{+}\right)}-\frac{1}{2} i \xi_{k}\right) \chi \\
\hat{\rho} \Rightarrow \hat{\rho} \hat{a}_{k} & \chi\left(\xi, \xi^{+}\right) \Rightarrow\left(\frac{\partial}{\partial\left(i \xi_{k}^{+}\right)}+\frac{1}{2} i \xi_{k}\right) \chi \\
\hat{\rho} \Rightarrow \hat{a}_{k}^{\dagger} \hat{\rho} & \chi\left(\xi, \xi^{+}\right) \Rightarrow\left(\frac{\partial}{\partial\left(i \xi_{k}\right)}+\frac{1}{2} i \xi_{k}^{+}\right) \chi \\
\hat{\rho} \Rightarrow \hat{\rho} \hat{a}_{k}^{\dagger} & \chi\left(\xi, \xi^{+}\right) \Rightarrow\left(\frac{\partial}{\partial\left(i \xi_{k}\right)}-\frac{1}{2} i \xi_{k}^{+}\right) \chi
\end{array}
$$

From these results the corresponding changes to the distribution functions can be deduced using (28) or (31). This involves integration by parts. The analyticity of the functions $\exp i \sum_{k=1}^{n}\left\{\xi_{k} \alpha_{k}^{+}\right\}$and $\exp i \sum_{k=1}^{n}\left\{\alpha_{k} \xi_{k}^{+}\right\}$enables two options for partial differentiation ( $\alpha_{k x}$ or $i \alpha_{k y}$ and $\alpha_{k x}^{+}$or $i \alpha_{k y}^{+}$), to be obtained, but this need not be made explicit here (see [42, [43] or [14]).

The correspondence rules for the non-condensate annihilation, creation operators are

$$
\begin{aligned}
& \hat{\rho} \Rightarrow \hat{a}_{k} \hat{\rho} \quad P\left(\alpha, \alpha^{+}, \alpha^{*}, \alpha^{+*}\right) \Rightarrow \alpha_{k} P \\
& \hat{\rho} \Rightarrow \hat{\rho} \hat{a}_{k} \quad P\left(\alpha, \alpha^{+}, \alpha^{*}, \alpha^{+*}\right) \Rightarrow\left(-\frac{\partial}{\partial \alpha_{k}^{+}}+\alpha_{k}\right) P \\
& \hat{\rho} \Rightarrow \hat{a}_{k}^{\dagger} \hat{\rho} \quad P\left(\alpha, \alpha^{+}, \alpha^{*}, \alpha^{+*}\right) \Rightarrow\left(-\frac{\partial}{\partial \alpha_{k}}+\alpha_{k}^{+}\right) P \\
& \hat{\rho} \Rightarrow \hat{\rho} \hat{a}_{k}^{\dagger} \quad P\left(\alpha, \alpha^{+}, \alpha^{*}, \alpha^{+*}\right) \Rightarrow \alpha_{k}^{+} P
\end{aligned}
$$

The correspondence rules for the condensate annihilation, creation operators are:

$$
\begin{array}{ccc}
\hat{\rho} \Rightarrow \hat{a}_{k} \hat{\rho} & P\left(\alpha, \alpha^{+}, \alpha^{*}, \alpha^{+*}\right) \Rightarrow\left(\alpha_{k}+\frac{1}{2} \frac{\partial}{\partial \alpha_{k}^{+}}\right) P \\
\hat{\rho} \Rightarrow \hat{\rho} \hat{a}_{k} & P\left(\alpha, \alpha^{+}, \alpha^{*}, \alpha^{+*}\right) \Rightarrow\left(\alpha_{k}-\frac{1}{2} \frac{\partial}{\partial \alpha_{k}^{+}}\right) P \\
\hat{\rho} \Rightarrow \hat{a}_{k}^{\dagger} \hat{\rho} & P\left(\alpha, \alpha^{+}, \alpha^{*}, \alpha^{+*}\right) \Rightarrow\left(\alpha_{k}^{+}-\frac{1}{2} \frac{\partial}{\partial \alpha_{k}}\right) P \\
\hat{\rho} \Rightarrow \hat{\rho} \hat{a}_{k}^{\dagger} & P\left(\alpha, \alpha^{+}, \alpha^{*}, \alpha^{+*}\right) \Rightarrow\left(\alpha_{k}^{+}+\frac{1}{2} \frac{\partial}{\partial \alpha_{k}}\right) P
\end{array}
$$

where the $\alpha, \alpha^{+}, \alpha^{*}, \alpha^{+*}$ are regarded as four independent complex variables.

By applying the correspondence rules (49) or (50) in succession to the various products of the density operator with mode annihilation, creation operators that arise from the $\frac{\partial}{\partial t} \hat{\rho}$ term the second term on the right hand side of Eq. (44) can 
be written as

$$
\begin{aligned}
& \chi\left(\xi, \xi^{+} ; \frac{\partial}{\partial t} \hat{\rho}\right) \\
= & \iint d^{2} \alpha d^{2} \alpha^{+} \exp \left(i \sum_{k \epsilon C, N C}\left(\alpha_{k} \xi_{k}^{+}+\xi_{k} \alpha_{k}^{+}\right)\right)\left\{-\sum_{k}\left(\frac{\partial}{\partial \alpha_{k}} A_{k}^{-}+\frac{\partial}{\partial \alpha_{k}^{+}} A_{k}^{+}\right)\right\} \\
& \times P\left(\alpha, \alpha^{+}, \alpha^{*}, \alpha^{+*}\right. \\
& +\iint d^{2} \alpha d^{2} \alpha^{+} \exp \left(i \sum_{k \epsilon C, N C}\left(\alpha_{k} \xi_{k}^{+}+\xi_{k} \alpha_{k}^{+}\right)\right) \\
& \times\left\{\frac{1}{2} \sum_{k} \sum_{l}\left(\frac{\partial}{\partial \alpha_{k}} \frac{\partial}{\partial \alpha_{l}} D_{k l}^{-}-\frac{\partial}{\partial \alpha_{k}^{+}} \frac{\partial}{\partial \alpha_{l}} D_{k l}^{+-}+\frac{\partial}{\partial \alpha_{k}} \frac{\partial}{\partial \alpha_{l}^{+}} D_{k l}^{-}+\frac{\partial}{\partial \alpha_{k}^{+}} \frac{\partial}{\partial \alpha_{l}^{+}} D_{k l}^{++}\right)\right\} \\
& \times P\left(\alpha, \alpha^{+}, \alpha^{*}, \alpha^{+*}\right)
\end{aligned}
$$

To obtain the specific forms of the drift $A_{k}^{-}, A_{k}^{+}$and diffusion terms $D_{k l}^{--}, D_{k l}^{+-}, D_{k l}^{-+}, D_{k l}^{++}$ the Liouville-von Neumann equation

$$
i \hbar \frac{\partial}{\partial t} \hat{\rho}=[\widehat{H}, \widehat{\rho}]
$$

is used in conjunction with the correspondence rules, with the Hamiltonian obtained from Eq. (2) by expanding the field operators in terms of mode annihilation, creation operators using Eqs. (8). The Hamiltonian will then contain terms of the form $\hat{a}_{k}^{\dagger} \hat{a}_{l}$ involving one mode creation operator and one mode annihilation operator from the kinetic and trap potential energies, as well as terms of the form $\hat{a}_{k}^{\dagger} \hat{a}_{l}^{\dagger} \hat{a}_{m} \hat{a}_{n}$ involving two mode creation operators and two mode annihilation operators from the boson-boson interaction energy. These terms will be multiplied by spatial integrals involving the mode functions - the latter being assumed known (see Section 2.4). Division of the modes as condensate and non-condensate is needed to identify whether Wigner or positive $\mathrm{P}$ correspondence rules apply. For the condensate modes, third order derivatives occur in the Fokker-Planck equation, but these are discarded as being too small to matter because of the large occupancy of the condensate modes. It is well known that there will only be first and second order derivatives associated with the non-condensate modes, these being associated with the positive $\mathrm{P}$ distribution.

We also need to consider the time derivative of the characteristic function in the form Eq. (31). Differentiation under the phase space integral can be carried out without any issues associated with time dependent differentials, as Eq. (40) shows. Also we see that similar to the proof of (42)

$$
\frac{\partial}{\partial t} \sum_{k} \alpha_{k} \xi_{k}^{+}=\frac{\partial}{\partial t} \sum_{k} \xi_{k} \alpha_{k}^{+}=\frac{\partial}{\partial t} \sum_{k}\left(\alpha_{k} \xi_{k}^{+}+\xi_{k} \alpha_{k}^{+}\right)=0
$$

Hence we have

$$
\frac{\partial}{\partial t} \chi\left(\xi, \xi^{+}\right)=\iint d^{2} \alpha d^{2} \alpha^{+} \exp \left(i \sum_{k \in C, N C}\left(\alpha_{k} \xi_{k}^{+}+\xi_{k} \alpha_{k}^{+}\right)\right) \frac{\partial}{\partial t} P\left(\alpha, \alpha^{+}, \alpha^{*}, \alpha^{+*}\right)
$$


Substituting the results from (54), (45) and (51) into Eq.(44) the FokkerPlanck equation for the distribution function can be obtained in the form

$$
\begin{aligned}
& \frac{\partial}{\partial t} P\left(\alpha, \alpha^{+}, \alpha^{*}, \alpha^{+*}\right) \\
= & -\sum_{k}\left(\frac{\partial}{\partial \alpha_{k}} A_{k}^{-}+\frac{\partial}{\partial \alpha_{k}^{+}} A_{k}^{+}\right) P\left(\alpha, \alpha^{+}, \alpha^{*}, \alpha^{+*}\right) \\
& +\frac{1}{2} \sum_{k} \sum_{l}\left(\frac{\partial}{\partial \alpha_{k}} \frac{\partial}{\partial \alpha_{l}} D_{k l}^{--}+\frac{\partial}{\partial \alpha_{k}^{+}} \frac{\partial}{\partial \alpha_{l}} D_{k l}^{+-}\right) P\left(\alpha, \alpha^{+}, \alpha^{*}, \alpha^{+*}\right) \\
& +\frac{1}{2} \sum_{k} \sum_{l}\left(\frac{\partial}{\partial \alpha_{k}} \frac{\partial}{\partial \alpha_{l}^{+}} D_{k l}^{-}+\frac{\partial}{\partial \alpha_{k}^{+}} \frac{\partial}{\partial \alpha_{l}^{+}} D_{k l}^{++}\right) P\left(\alpha, \alpha^{+}, \alpha^{*}, \alpha^{+*}\right) \\
& +\frac{1}{2} \sum_{k \in C} \sum_{l \in N C}\left(C_{k l} \frac{\partial}{\partial \alpha_{l}^{+}} \frac{\partial}{\partial \alpha_{k}}+C_{k l}^{*} \frac{\partial}{\partial \alpha_{k}^{+}} \frac{\partial}{\partial \alpha_{l}}\right) P\left(\alpha, \alpha^{+}, \alpha^{*}, \alpha^{+*}\right)
\end{aligned}
$$

As the expressions are now becoming rather lengthy we now change to a simpler notation.

\subsubsection{Notation Change}

We first introduce the symbol $A$ to designate condensate and non-condensate modes, where $A=C, N C$. The mode functions $\phi_{k}(x, t)$ and their conjugates $\phi_{k}^{*}(x, t)$ will now be designated $\phi_{A k}(x, t)$ and their conjugates $\phi_{A k}^{*}(x, t)$, where $k=1,2, . ., n_{A}$ lists the separate condensate or non-condensate modes. Both the $\phi_{A k}(x, t)$ and $\phi_{A k}^{*}(x, t)$ may finally be listed as $\phi_{A k}^{\mu}(x, t)$ with $\mu=-,+$, so that $\phi_{A k}^{-}(x, t)=\phi_{A k}(x, t), \phi_{A k}^{+}(x, t)=\phi_{A k}^{*}(x, t)$. Note that if there are $n_{C}$ condensate modes and $n_{N C}$ non-condensate modes, the total number of $\phi_{A k}^{\mu}(x, t)$ will be $2\left(n_{C}+n_{N C}\right)$, which is twice the total number of modes. This is because there are $n=n_{C}+n_{N C}$ modes $\phi_{k}(x, t)$ plus their complex conjugates $\phi_{k}^{*}(x, t)$. The phase space variables will now be written as $\alpha_{\mu A k}$ and those in the characteristic function as $\xi_{\mu A k}$. The equations for the phase space and the characteristic function variables now become

$$
\begin{aligned}
& \frac{\partial \alpha_{\mu A k}(t)}{\partial t}=\sum_{B l} C_{A k B l}^{\mu}(t) \alpha_{\mu B l}(t) \\
& \frac{\partial \xi_{\mu A k}(t)}{\partial t}=\sum_{B l} C_{A k B l}^{\mu}(t) \xi_{\mu B l}(t)
\end{aligned}
$$

with

$$
\begin{aligned}
C_{A k B l}^{-}(t) & =\int d x \frac{\partial \phi_{A k}^{*}(x, t)}{\partial t} \phi_{B l}(x, t)=C_{A k B l}(t) \\
C_{A k B l}^{+}(t) & =\int d x \frac{\partial \phi_{A k}(x, t)}{\partial t} \phi_{B l}^{*}(x, t)=C_{A k B l}^{*}(t) \\
C_{A k B l}^{\mu} & =-C_{B l A k}^{-\mu}
\end{aligned}
$$


Note that the sums in (56) and (57) include both condensate and non-condensate modes. The original drift, diffusion terms are now designated as $A_{A k}^{\mu}, D_{A k B l}^{\mu \nu}$. The time derivatives of the modes are now written as

$$
\frac{\partial}{\partial t} \phi_{A k}^{\mu}(x, t)=\sum_{B l} C_{A k B l}^{-\mu} \phi_{B l}^{\mu}(x, t)
$$

The orthonormality and completeness relationships will now be

$$
\begin{aligned}
\int d x \phi_{A k}^{-\mu}(x, t) \phi_{B l}^{\mu}(x, t) & =\delta_{A B} \delta_{k l} \\
\sum_{k} \phi_{A k}^{\mu}(x, t) \phi_{A k}^{-\mu}(y, t) & =\delta_{\mu A}(x, y)
\end{aligned}
$$

where $\delta_{\mu A}(x, y)$ is the restricted delta function for the $\mu A$ modes and the projectors $\mathcal{P}_{x}^{\mu A}$ now are

$$
\begin{aligned}
\mathcal{P}_{x}^{\mu A}[F(x)] & =\int d y \delta_{\mu A}(x, y) F(y) \\
\mathcal{P}_{x}^{\mu A}\left[\sum_{k} \beta_{k} \phi_{A k}^{\mu}(x, t)+\sum_{\substack{B l \\
B \neq A}} \beta_{l} \phi_{B l}^{\mu}(x, t)\right] & =\sum_{k} \beta_{k} \phi_{A k}^{\mu}(x, t)
\end{aligned}
$$

so that the projector $\mathcal{P}_{x}^{\mu A}$ acting on an arbitrary function written as a linear combination of the modes $\phi_{A k}^{\mu}(x, t)$ and orthogonal modes $\phi_{B l}^{\mu}(x, t)(B \neq A)$ projects the function onto just the linear combination of the modes $\phi_{A k}^{\mu}(x, t)$.

\subsubsection{Fokker-Planck Equation Result}

In terms of the new notation the Fokker-Planck equation will be of the form

$$
\begin{aligned}
& \frac{\partial}{\partial t} P\left(\alpha, \alpha^{+}, \alpha^{*}, \alpha^{+*}\right) \\
= & \left\{-\sum_{\mu A k} \frac{\partial}{\partial \alpha_{\mu A k}} A_{A k}^{\mu}+\frac{1}{2} \sum_{\mu A k} \sum_{\nu B l} \frac{\partial}{\partial \alpha_{\mu A k}} \frac{\partial}{\partial \alpha_{\nu B l}} E_{A k B l}^{\mu \nu}\right\} P\left(\alpha, \alpha^{+}, \alpha^{*}, \alpha^{+*}\right)
\end{aligned}
$$

where $A_{A k}^{\mu}$ is the drift vector and $E_{A k B l}^{\mu \nu}$ is the diffusion matrix. The diffusion matrix is given by

$$
E_{A k B l}^{\mu \nu}=D_{A k B l}^{\mu \nu}+\frac{1}{2}\left(\delta_{A C} \delta_{B N C} \delta_{\mu-\nu} C_{A k B l}^{\mu}+\delta_{B C} \delta_{A N C} \delta_{\nu-\mu} C_{B l A k}^{\nu}\right)
$$

The first term $D_{A k B l}^{\mu \nu}$ is from the standard derivation of the Fokker-Planck equation via applying the standard correspondence rules to the terms in the characteristic function that arise from $\frac{\partial}{\partial t} \hat{\rho}$, the second arises from the time 
dependence of the condensate and non-condensate mode functions. Note that the diffusion matrix is still symmetric

$$
E_{A k B l}^{\mu \nu}=E_{B l A k}^{\nu \mu}
$$

The drift $A_{A k}^{\mu}$ vector and the diffusion matrix $E_{A k B l}^{\mu \nu}$ are functions of the $\alpha_{\mu A k}$. In the present approach using time dependent phase variables the drift vector $A_{A k}^{\mu}$ vector is the same as those that would be obtained if time independent modes were used, but the diffusion matrix $E_{A k B l}^{\mu \nu}$ is changed from the expected $D_{A k B l}^{\mu \nu}$. The Fokker-Planck equation can also be expressed in terms of real variables which involve the real and imaginary components of the phase variables $\alpha_{\mu A k}$, but we will not do that here. In deriving the Fokker-Planck equation there are often terms involving third and higher order derivatives arising from the condensate modes and their Wigner representation. These are usually small, scaling as higher powers of $1 / \sqrt{N}$, and hence are discarded. Thus we see that the Fokker-Planck equation has changed in this hybrid approach from that for time independent modes.

\subsection{Langevin (Ito) Stochastic Equations}

In this section we determine the Langevin equations that are equivalent to the Fokker-Planck equation for the distribution function. These will be in the form of Ito stochastic differential equations. The derivation is based on that given by Gardiner [43, but modified to allow for the phase variables being time dependent. The final stochastic averages would determine normally ordered quantum correlation functions associated with non-condensate operators, or symmetrically ordered quantum correlation functions associated with condensate operators. Note also that the Langevin equation derivation does not depend on the distribution function having any particular properties, such as being real or positive. We must of course assume it to be non-analytic in general.

\subsubsection{Phase Space Average}

The phase space average of the functions, $F\left(\alpha, \alpha^{+}\right)$is given by

$$
\left\langle F\left(\alpha, \alpha^{+}\right)\right\rangle_{t}=\iint d^{2} \alpha^{+} d^{2} \alpha F\left(\alpha, \alpha^{+}\right) P\left(\alpha, \alpha^{+}, \alpha^{*}, \alpha^{+*}, t\right)
$$

For determining the quantum correlation function $G\left(l_{1}, l_{2}, . . l_{p} ; m_{q}, . ., m_{2}, m_{1}\right.$ :: $\left.j_{1}, j_{2}, . . j_{r} ; k_{s}, . ., k_{2}, k_{1}\right)$ in Eq.(35) the function is

$F\left(\alpha, \alpha^{+}\right)=\left[\alpha_{l_{1}}^{+} \alpha_{l_{2}}^{+} . . \alpha_{l_{p}}^{+} \alpha_{m_{q}} . \alpha_{m_{2}} \alpha_{m_{1}}\right]\left[\alpha_{j_{1}}^{+} \alpha_{j_{2}}^{+} . . \alpha_{j_{r}}^{+} \alpha_{k_{s}} . \alpha_{k_{2}} \alpha_{k_{1}}\right]$. The phase space average will change with time not only because the distribution function $P\left(\alpha, \alpha^{+}, \alpha^{*}, \alpha^{+*}, t\right)$ is time dependent, but also because $F\left(\alpha, \alpha^{+}\right)$is now time dependent since from Eq.(37) the $\alpha, \alpha^{+}$depend on time. Note the differentials $\iint d^{2} \alpha^{+} d^{2} \alpha$ are time independent. 
The change in the phase space average from times $t$ to $t+\delta t$ due to all factors is given by

$$
\begin{aligned}
& \left\langle F\left(\alpha, \alpha^{+}\right)\right\rangle_{t+\delta t}-\left\langle F\left(\alpha, \alpha^{+}\right)\right\rangle_{t} \\
= & \left(\iint d^{2} \alpha^{+} d^{2} \alpha F\left(\alpha, \alpha^{+}\right)\left\{\frac{\partial}{\partial t} P\left(\alpha, \alpha^{+}, \alpha^{*}, \alpha^{+*}, t\right)\right\}\right) \delta t \\
+ & \left(\iint d^{2} \alpha^{+} d^{2} \alpha\left\{\frac{\partial}{\partial t} F\left(\alpha, \alpha^{+}\right)\right\} P\left(\alpha, \alpha^{+}, \alpha^{*}, \alpha^{+*}, t\right)\right) \delta t
\end{aligned}
$$

The second term in the last equation is absent in the standard treatment 43 ] based on time independent phase variables. This result gives the change in the phase space average correct to $O(\delta t)$.

Now from (56)

$$
\begin{aligned}
\frac{\partial}{\partial t} F\left(\alpha, \alpha^{+}\right) & =\sum_{\mu A k} \frac{\partial}{\partial \alpha_{\mu A k}} F\left(\alpha, \alpha^{+}\right) \frac{\partial \alpha_{\mu A k}}{\partial t} \\
& =\sum_{\mu A k} \frac{\partial}{\partial \alpha_{\mu A k}} F\left(\alpha, \alpha^{+}\right) \sum_{B l} C_{A k B l}^{\mu} \alpha_{\mu B l}
\end{aligned}
$$

Substituting for $\frac{\partial}{\partial t} P$ from the Fokker-Planck equation (63) and for $\frac{\partial}{\partial t} F$ from (68) we get using integration by parts and assuming that the distribution function goes to zero fast enough on the phase space boundary

$$
\begin{aligned}
& \left\langle F\left(\alpha, \alpha^{+}\right)\right\rangle_{t+\delta t}-\left\langle F\left(\alpha, \alpha^{+}\right)\right\rangle_{t} \\
= & \iint d^{2} \alpha^{+} d^{2} \alpha\left\{\sum_{\mu A k}\left[\frac{\partial}{\partial \alpha_{\mu A k}} F\left(\alpha, \alpha^{+}\right)\right]\left[A_{A k}^{\mu}+\sum_{B l} C_{A k B l}^{\mu} \alpha_{\mu B l}\right]\right\} \\
& \times P\left(\alpha, \alpha^{+}, \alpha^{*}, \alpha^{+*}, t\right) \delta t \\
& +\iint d^{2} \alpha^{+} d^{2} \alpha\left\{\frac{1}{2} \sum_{\mu A k} \sum_{\nu B l}\left[\frac{\partial}{\partial \alpha_{\mu A k}} \frac{\partial}{\partial \alpha_{\nu B l}} F\left(\alpha, \alpha^{+}\right)\right] E_{A k B l}^{\mu \nu}\right\} \\
& \times P\left(\alpha, \alpha^{+}, \alpha^{*}, \alpha^{+*}, t\right) \delta t
\end{aligned}
$$

where the terms involving first order derivatives have been combined.

Hence

$$
\begin{aligned}
& \frac{d}{d t}\left\langle F\left(\alpha, \alpha^{+}\right)\right\rangle_{t} \\
= & \left\langle\left\{\sum_{\mu A k}\left[\frac{\partial}{\partial \alpha_{\mu A k}} F\left(\alpha, \alpha^{+}\right)\right]\left[A_{A k}^{\mu}+\sum_{B l} C_{A k B l}^{\mu} \alpha_{\mu B l}\right]\right\}\right\rangle \\
+ & \left\langle\left\{\frac{1}{2} \sum_{\mu A k} \sum_{\nu B l}\left[\frac{\partial}{\partial \alpha_{\mu A k}} \frac{\partial}{\partial \alpha_{\nu B l}} F\left(\alpha, \alpha^{+}\right)\right] E_{A k B l}^{\mu \nu}\right\}\right\rangle
\end{aligned}
$$


giving the time derivative of the phase space average of $F\left(\alpha, \alpha^{+}\right)$as the phase space average of the sum of the two quantities in the \{\} brackets. Note that no specific properties of the distribution function were needed. This result will be used later to derive results for time derivatives of quantum correlation functions.

\subsubsection{Stochastic Variables Average}

For the Ito stochastic approach we now replace the variables $\alpha_{\mu A k}$ by stochastic variables $\alpha_{\mu A k}^{s}$. In terms of these stochastic variables the stochastic average at time $t$ of $F\left(\alpha, \alpha^{+}\right)$is given by

$$
\overline{F\left(\alpha^{s}(t), \alpha^{s+}(t)\right)}=\frac{1}{N} \sum_{i=1}^{N} f\left(\alpha_{\mu A k i}^{s}(t)\right)
$$

where $\alpha_{\mu A k i}^{s}(t)$ is the $i$ th member of the stochastic ensemble of $N$ samples and $f\left(\alpha_{\mu A k}^{s}(t)\right)$ is the same as $F\left(\alpha^{s}(t), \alpha^{s+}(t)\right)$. The key idea is that the phase space average at any time $t$ of arbitrary functions $F\left(\alpha, \alpha^{+}\right)$and the stochastic average of such functions are made to coincide when the stochastic equation for the $\alpha_{\mu A k}^{s}(t)$ is suitably related to the Fokker-Planck equation for the distribution function $P\left(\alpha, \alpha^{+}, \alpha^{*}, \alpha^{+*}, t\right)$. Thus

$$
\begin{aligned}
\left\langle F\left(\alpha, \alpha^{+}\right)\right\rangle_{t} & =\iint d^{2} \alpha^{+} d^{2} \alpha F\left(\alpha, \alpha^{+}\right) P\left(\alpha, \alpha^{+}, \alpha^{*}, \alpha^{+*}, t\right) \\
& =\frac{F\left(\alpha^{s}(t), \alpha^{s+}(t)\right)}{N} \\
& =\frac{1}{N} \sum_{i=1}^{N} f\left(\alpha_{\mu A k i}^{s}(t)\right)
\end{aligned}
$$

In turn, the phase space averages are related to quantum averages. In particular the normally ordered quantum correlation functions are given by the stochastic average of the product of stochastic c-number phase space variables

$$
\left\langle\widehat{a}_{l_{1}}^{\dagger} \widehat{a}_{l_{2}}^{\dagger} . . \widehat{a}_{l_{p}}^{\dagger} \widehat{a}_{m_{q}} . . \widehat{a}_{m_{2}} \widehat{a}_{m_{1}}\right\rangle_{t}=\overline{\left(\alpha_{m_{q}}^{s}(t) . \alpha_{m_{1}}^{s}(t)\right)\left(\alpha_{l_{1}}^{+s}(t) . . \alpha_{l_{p}}^{+s}(t)\right)}
$$

For bosonic systems such stochastic averages involving c-numbers can be carried out numerically, and this method is often more efficient than having to determine the full distribution function. . 
Now the difference in the stochastic average from time $t$ to $t+\delta t$ is given by

$$
\begin{aligned}
= & \frac{\overline{F\left(\alpha^{s}(t+\delta t), \alpha^{s+}(t+\delta t)\right)}-\overline{F\left(\alpha^{s}(t), \alpha^{s+}(t)\right)}}{\left\{F\left(\alpha^{s}(t+\delta t), \alpha^{s+}(t+\delta t)\right)-F\left(\alpha^{s}(t), \alpha^{s+}(t)\right)\right\}} \\
= & \frac{\left\{f\left(\alpha_{\mu A k i}^{s}(t+\delta t)\right)-f\left(\alpha_{\mu A k i}^{s}(t)\right)\right\}}{\left\{\sum_{\mu A k}\left[\frac{\partial}{\partial \alpha_{\mu A k}} F\left(\alpha, \alpha^{+}\right)\right] \delta \alpha_{\mu A k}^{s}(t)\right\}} \\
& +\left\{\frac{1}{2} \sum_{\mu A k} \sum_{\nu B l}\left[\frac{\partial}{\partial \alpha_{\mu A k}} \frac{\partial}{\partial \alpha_{\nu B l}} F\left(\alpha, \alpha^{+}\right)\right] \delta \alpha_{\mu A k}^{s}(t) \delta \alpha_{\nu B l}^{s}(t)+. .\right\}
\end{aligned}
$$

where we have used a Taylor expansion for $f\left(\alpha_{\mu A k}^{s}(t+\delta t)\right)$ with the notation

$$
\delta \alpha_{\mu A k}^{s}(t)=\alpha_{\mu A k}^{s}(t+\delta t)-\alpha_{\mu A k}^{s}(t)
$$

for the fluctuation in $\alpha_{\mu A k}^{s}$. In obtaining the last result the stochastic average of a sum being equal to the sum of the stochastic averages has been used. It is necessary to consider fluctuations of the second order because - as we will see stochastic averages of such terms are of $O(\delta t)$.

Now suppose $\alpha_{\mu A k}^{s}(t)$ satisfies an Ito stochastic equation of the form

$$
\begin{aligned}
& \alpha_{\mu A k}^{s}(t+\delta t)-\alpha_{\mu A k}^{s}(t) \\
= & \mathcal{A}_{A k}^{\mu}\left(\alpha_{\xi D m}^{s}(t)\right) \delta t+\sum_{a} \mathcal{B}_{A k a}^{\mu}\left(\alpha_{\xi D m}^{s}(t)\right) \int_{t}^{t+\delta t} d t_{1} \Gamma_{a}\left(t_{1}\right)
\end{aligned}
$$

which gives the change in $\alpha_{\mu A k}^{s}(t)$ correct to $O(\delta t)$. The Ito stochastic equation is also written as a differential equation

$$
\frac{\partial}{\partial t} \alpha_{\mu A k}^{s}=\mathcal{A}_{A k}^{\mu}\left(\alpha_{\xi D m}^{s}(t)\right)+\sum_{a} \mathcal{B}_{A k a}^{\mu}\left(\alpha_{\xi D m}^{s}(t)\right) \Gamma_{a}\left(t_{+}\right)
$$

where the stochastic behaviour is due to the Gaussian-Markoff random noise terms $\Gamma_{a}$. The aim is to find expressions for the $\mathcal{A}_{A k}^{\mu}$ and the $\mathcal{B}_{A k a}^{\mu}$ so that the phase space and stochastic averages coincide for an arbitrary choice of $F\left(\alpha, \alpha^{+}\right)$.

The $\Gamma_{a}(t)$ are real Gaussian-Markoff random noise terms $(a=1,2, .$.$) whose$ stochastic averages are given by

$$
\begin{aligned}
\overline{\Gamma_{a}\left(t_{1}\right)}= & 0 \\
\overline{\Gamma_{a}\left(t_{1}\right) \Gamma_{b}\left(t_{2}\right)}= & \delta_{a b} \delta\left(t_{1}-t_{2}\right) \\
\overline{\Gamma_{a}\left(t_{1}\right) \Gamma_{b}\left(t_{2}\right) \Gamma_{c}\left(t_{3}\right)}= & 0 \\
\overline{\Gamma_{a}\left(t_{1}\right) \Gamma_{b}\left(t_{2}\right) \Gamma_{c}\left(t_{3}\right) \Gamma_{d}\left(t_{4}\right)}= & \left.\overline{\Gamma_{a}\left(t_{1}\right) \Gamma_{b}\left(t_{2}\right)} \overline{\Gamma_{c}\left(t_{3}\right) \Gamma_{d}\left(t_{4}\right.}\right)+\overline{\Gamma_{a}\left(t_{1}\right) \Gamma_{c}\left(t_{3}\right)} \overline{\Gamma_{b}\left(t_{2}\right) \Gamma_{d}\left(t_{4}\right)} \\
& +\overline{\Gamma_{a}\left(t_{1}\right) \Gamma_{d}\left(t_{4}\right)} \overline{\Gamma_{b}\left(t_{2}\right) \Gamma_{c}\left(t_{3}\right)}
\end{aligned}
$$$$
\text { ... }
$$ 
so that the stochastic averages of products of odd numbers of $\Gamma$ are zero and the stochastic averages of products of even numbers of $\Gamma$ are the sums of products of stochastic averages of pairs of $\Gamma$. For the moment we leave the number of $\Gamma_{a}$ unspecified, the number will turn out to be $2\left(n_{C}+n_{N C}\right)$. It is also assumed that any function $h$ of the $\alpha_{\mu A k}^{s}(t)$ at time $t$ and the $\Gamma_{a}(t)$ at later times are uncorrelated - this is the meaning of the $\Gamma_{a}\left(t_{+}\right)$in (77). Thus

$$
=\overline{\overline{h\left(\alpha_{\mu A k}^{s}\left(t_{1}\right)\right) \Gamma_{a}\left(t_{2}\right) \Gamma_{b}\left(t_{3}\right) \Gamma_{c}\left(t_{4}\right) . . \Gamma_{k}\left(t_{l}\right)}} \quad \frac{}{h\left(\alpha_{\mu A k}^{s}\left(t_{1}\right)\right)} \frac{t_{1}<t_{2}, t_{3}, . ., t_{l}}{\Gamma_{a}\left(t_{2}\right) \Gamma_{b}\left(t_{3}\right) \Gamma_{c}\left(t_{4}\right) . . \Gamma_{k}\left(t_{l}\right)} \quad t
$$

With these results we can now obtain expressions for the stochastic averages in Eq.(74). Details are given in Appendix 8.

For the first order derivative terms we find that

$$
\begin{aligned}
& \overline{\left\{\sum_{\mu A k}\left[\frac{\partial}{\partial \alpha_{\mu A k}} F\left(\alpha, \alpha^{+}\right)\right] \delta \alpha_{\mu A k}^{s}(t)\right\}} \\
& =\overline{\sum_{\mu A k}\left[\frac{\partial}{\partial \alpha_{\mu A k}} F\left(\alpha, \alpha^{+}\right)\right] \mathcal{A}_{A k}^{\mu}\left(\alpha_{\xi D m}^{s}(t)\right)} \delta t
\end{aligned}
$$

where the stochastic average rules for sums and products have been used, the non-correlation between the averages of functions of $\alpha_{\mu A k}^{s}(t)$ at time $t$ and the $\Gamma$ at later times between $t$ to $t+\delta t$ is applied, and the term involving $\overline{\Gamma_{a}\left(t_{1}\right)}$ is equal to zero from (78). Note that the first order term is proportional to $\delta t$.

For the second order derivative terms, we have on expanding the product $\delta \alpha_{\mu A k}^{s}(t) \delta \alpha_{\nu B l}^{s}(t)$, using the stochastic average of a sum being the same as the sum of stochastic averages, the result that the stochastic averages for the functions of the $\alpha_{\xi D m}^{s}(t)$ and the $\Gamma_{a}\left(t_{+}\right)$are uncorrelated, and after using the result that terms $\overline{\Gamma_{a}\left(t_{1}\right)}$ involving single $\Gamma^{\prime} s$ give zero together with the result for terms $\overline{\Gamma_{a}\left(t_{1}\right) \Gamma_{b}\left(t_{2}\right)}$ involving two $\Gamma^{\prime} s$

$$
\begin{aligned}
\overline{\int_{t}^{t+\delta t} d t_{1} \Gamma_{a}\left(t_{1}\right) \int_{t}^{t+\delta t} d t_{2} \Gamma_{b}\left(t_{2}\right)} & =\int_{t}^{t+\delta t} d t_{1} \int_{t}^{t+\delta t} d t_{2} \overline{\Gamma_{a}\left(t_{1}\right) \Gamma_{b}\left(t_{2}\right)} \\
& =\int_{t}^{t+\delta t} d t_{1} \int_{t}^{t+\delta t} d t_{2} \delta_{a b} \delta\left(t_{1}-t_{2}\right) \\
& =\delta_{a b} \delta t
\end{aligned}
$$


we find that the second order derivative term is

$$
\begin{aligned}
& \overline{\left\{\frac{1}{2} \sum_{\mu A k} \sum_{\nu B l}\left[\frac{\partial}{\partial \alpha_{\mu A k}} \frac{\partial}{\partial \alpha_{\nu B l}} F\left(\alpha, \alpha^{+}\right)\right] \delta \alpha_{\mu A k}^{s}(t) \delta \alpha_{\nu B l}^{s}(t)\right\}} \\
& =\overline{\frac{1}{2} \sum_{\mu A k} \sum_{\nu B l}\left[\frac{\partial}{\partial \alpha_{\mu A k}} \frac{\partial}{\partial \alpha_{\nu B l}} F\left(\alpha, \alpha^{+}\right)\right]\left[\sum_{a} \mathcal{B}_{A k a}^{\mu}\left(\alpha_{\xi D m}^{s}(t)\right) \mathcal{B}_{B l a}^{\nu}\left(\alpha_{\xi D m}^{s}(t)\right)\right]} \delta t \\
& =\overline{\frac{1}{2} \sum_{\mu A k} \sum_{\nu B l}\left[\frac{\partial}{\partial \alpha_{\mu A k}} \frac{\partial}{\partial \alpha_{\nu B l}} F\left(\alpha, \alpha^{+}\right)\right]\left[\left[\mathcal{B}\left(\alpha_{\xi D m}^{s}(t)\right) \mathcal{B}^{T}\left(\alpha_{\xi D m}^{s}(t)\right)\right]_{A k, B l}^{\mu, \nu}\right]} \delta t
\end{aligned}
$$

after neglecting terms of order $\delta t^{2}$ Note that the second order term is also proportional to $\delta t$

The remaining terms give stochastic averages correct to order $\delta t^{2}$ or higher so that we have correct to first order in $\delta t$

$$
\begin{aligned}
& =\overline{F\left(\alpha^{s}(t+\delta t), \alpha^{s+}(t+\delta t)\right)}-\overline{F\left(\alpha^{s}(t), \alpha^{s+}(t)\right)} \\
& =\left\{\overline{\sum_{\mu A k}\left[\frac{\partial}{\partial \alpha_{\mu A k}} F\left(\alpha, \alpha^{+}\right)\right] \mathcal{A}_{A k}^{\mu}\left(\alpha_{\xi D m}^{s}(t)\right)}\right\} \delta t \\
& +\left\{\overline{\frac{1}{2} \sum_{\mu A k} \sum_{\nu B l}\left[\frac{\partial}{\partial \alpha_{\mu A k}} \frac{\partial}{\partial \alpha_{\nu B l}} F\left(\alpha, \alpha^{+}\right)\right]\left[\left[\mathcal{B}\left(\alpha_{\xi D m}^{s}(t)\right) \mathcal{B}^{T}\left(\alpha_{\xi D m}^{s}(t)\right)\right]_{A k, B l}^{\mu, \nu}\right]}\right\} \delta t
\end{aligned}
$$

or

$$
\begin{aligned}
& \frac{d}{d t} \overline{F\left(\alpha^{s}(t), \alpha^{s+}(t)\right)} \\
& =\overline{\sum_{\mu A k}\left[\frac{\partial}{\partial \alpha_{\mu A k}} F\left(\alpha, \alpha^{+}\right)\right] \mathcal{A}_{A k}^{\mu}\left(\alpha_{\xi D m}^{s}(t)\right)} \\
& +\overline{\frac{1}{2} \sum_{\mu A k} \sum_{\nu B l}\left[\frac{\partial}{\partial \alpha_{\mu A k}} \frac{\partial}{\partial \alpha_{\nu B l}} F\left(\alpha, \alpha^{+}\right)\right]\left[\left[\mathcal{B}\left(\alpha_{\xi D m}^{s}(t)\right) \mathcal{B}^{T}\left(\alpha_{\xi D m}^{s}(t)\right)\right]_{A k, B l}^{\mu, \nu}\right]}
\end{aligned}
$$

This result is exactly the same as for the standard treatment based on time independent mode functions, since the Ito stochastic equations (177) have exactly the same form in that situation.

The result (84) for the stochastic average will be the same as that in (70) based on the phase space average if we have the following relationships between the matrices $A$ and $E$ in the Fokker-Planck equation and the matrices $\mathcal{A}$ and $\mathcal{B}$ 
occurring in the Ito stochastic differential equation.

$$
\begin{aligned}
\mathcal{A}_{A k}^{\mu} & =A_{A k}^{\mu}+\sum_{B l} C_{A k B l}^{\mu} \alpha_{\mu B l} \\
{\left[\mathcal{B} \mathcal{B}^{T}\right]_{A k, B l}^{\mu, \nu} } & =E_{A k B l}^{\mu \nu}
\end{aligned}
$$

If the modes were time independent, the coupling constants would all be zero and the relationship would have been $\mathcal{A}_{A k}^{\mu}=A_{A k}^{\mu}$ and $\left[\mathcal{B} \mathcal{B}^{T}\right]_{A k, B l}^{\mu, \nu}=D_{A k B l}^{\mu \nu}$, which relates the Ito equation quantities $\mathcal{A}$ and $\mathcal{B}$ to the $\operatorname{drift} A$ and diffusion $D$ terms that occur in the standard Fokker-Planck equation obtained from just the $\frac{\partial}{\partial t} \hat{\rho}$ term in the time derivative of the characteristic function. This is the usual condition found in the textbooks [43]. For the hybrid case with time dependent modes not only is the relationship changed for the diffusion terms, but it is also changed for the drift terms. The difference is entirely due to the presence of the coupling terms $C_{A k B l}^{\mu}$ that depend on the time dependences of the modes $\phi_{A k}^{\mu}(x, t)$. Clearly, if the drift vector $A$ from the Fokker-Planck equation and the matrix $C$ from the time dependence of the mode functions are known then the vector $\mathcal{A}$ in the Ito stochastic equation can be found. It is known 42 that the complex symmetric matrix $E$ may be factorised in the form $K K^{T}=E$, so the matrix $\mathcal{B}$ in the Ito stochastic equation can also be determined.

$$
\begin{aligned}
\mathcal{B} & =K \\
K K^{T} & =E
\end{aligned}
$$

This result is known as the Takagi factorisation [45. The proof is given in [46] (see section 4.3). The construction involves the eigenvectors of the matrix $E E^{*}$, which is hermitian because $E$ is symmetric, and which also has non-negative real eigenvalues This result is not well-known, and does not require $E$ to be positive semi-definite, as is sometimes thought to be the case. Note that in general $\mathcal{B}$ is a complex $2 n \times 2 n$ matrix. Also note that $\mathcal{B}$ is not unique, since with any orthogonal matrix $\mathcal{R}$ we also have $(\mathcal{B R})(\mathcal{B R})^{T}=E$. Again, the total number of Gaussian-Markoff noise terms $\Gamma_{a}\left(t_{+}\right)$is $2 n$, the total number of $\alpha_{A k}$ and $\alpha_{A k}^{+}$, or equivalently twice the total number of modes.

\subsection{Expressions for Ito Stochastic Equations}

We can also write the Ito stochastic equation (77) in terms of quantities that appear in the Fokker-Planck equation. From Eq. (85) we have for the Ito stochastic phase variable equation in the hybrid case

$$
\frac{\partial}{\partial t} \alpha_{\mu A k}^{s}=A_{A k}^{\mu}+\sum_{l} C_{A k B l}^{\mu} \alpha_{\mu B l}^{s}+\sum_{a} K_{A k a}^{\mu} \Gamma_{a}\left(t_{+}\right)
$$

where $A$ is the drift vector and $K$ is related to the diffusion matrix $E$ via

$$
\begin{aligned}
\sum_{a} K_{A k a}^{\mu} K_{B l a}^{\nu} & =E_{A k B l}^{\mu \nu} \\
& =D_{A k B l}^{\mu \nu}+\frac{1}{2}\left(\delta_{A C} \delta_{B N C} \delta_{\mu-\nu} C_{A k B l}^{\mu}+\delta_{B C} \delta_{A N C} \delta_{\nu-\mu} C_{B l A k}^{\nu}\right)
\end{aligned}
$$


This form of the Ito equation is the most useful as it involves the drift, diffusion terms in the Fokker-Planck equation (63) plus the coupling coefficients that allow for the modes being time dependent. Note that the diffusion terms (64) in the Fokker-Planck equation also involve the coupling constants. Thus the equivalence between the quantities $\mathcal{A}, \mathcal{B}$ in the Ito stochastic equations and the drift $A$, diffusion $D$ terms in the standard Fokker-Planck equation and the mode time dependence matrix $C$ can be established. There is an extra term $\sum_{l} C_{A k B l}^{\mu} \alpha_{\mu B l}^{s}$ (which is linear in the phase variables) in the drift term, and an extra term $\frac{1}{2}\left(\delta_{A C} \delta_{B N C} \delta_{\mu-\nu} C_{A k B l}^{\mu}+\delta_{B C} \delta_{A N C} \delta_{\nu-\mu} C_{B l A k}^{\nu}\right)$ (which is independent of the phase variables) in the diffusion term, which are not present in the standard treatment involving time independent modes.

\subsection{Classical and Noise Terms}

We can write the Ito stochastic equation in terms of a classical term and a noise term

$$
\begin{aligned}
\frac{\partial}{\partial t} \alpha_{\mu A k}^{s} & =\left(\frac{\partial}{\partial t} \alpha_{\mu A k}^{s}\right)_{\text {class }}+\left(\frac{\partial}{\partial t} \alpha_{\mu A k}^{s}\right)_{n o i s e} \\
\left(\frac{\partial}{\partial t} \alpha_{\mu A k}^{s}\right)_{\text {class }} & =A_{A k}^{\mu}+\sum_{l} C_{A k B l}^{\mu} \alpha_{\mu B l}^{s} \\
\left(\frac{\partial}{\partial t} \alpha_{\mu A k}^{s}\right)_{n o i s e} & =\sum_{a} K_{A k a}^{\mu} \Gamma_{a}\left(t_{+}\right)
\end{aligned}
$$

If only the classical terms were included, then the solution for $\alpha_{\mu A k}^{s}$ would determine classical trajectories in phase space; hence their name.

\subsection{Properties of Noise}

The stochastic averages of the noise terms can now be evaluated. For a single noise term and the product of two noise terms we have using Eqs. (78) and (88)

$$
\begin{aligned}
\overline{\left(\frac{\partial}{\partial t} \alpha_{\mu A k}^{s}(t)\right)_{n}} & =0 \\
\overline{\left(\frac{\partial}{\partial t} \alpha_{\mu A k}^{s}\left(t_{1}\right)\right)_{n}\left(\frac{\partial}{\partial t} \alpha_{\nu B l}^{s}\left(t_{2}\right)\right)_{n}} & =\delta\left(t_{1}-t_{2}\right) \overline{E_{A k B l}^{\mu \nu}\left(t_{1,2}\right)}
\end{aligned}
$$

showing that the stochastic average of a single phase noise term is zero whilst that for the product of two phase noise terms is delta correlated in time and equal to the appropriate diffusion matrix element.

In fact the diffusion matrix elements determine all the stochastic averages of products of phase noise terms. With an odd number of terms the stochastic average is zero. For an even number of terms the stochastic average involves sums of stochastic averages of products of diffusion matrix elements, which 
reflects the Gaussian-Markoff properties of the $\Gamma_{a}$. Thus for three and four phase noise terms

$$
\begin{aligned}
& \overline{\left(\frac{\partial}{\partial t} \alpha_{\mu A k}^{s}\left(t_{1}\right)\right)_{n}\left(\frac{\partial}{\partial t} \alpha_{\nu B l}^{s}\left(t_{2}\right)\right)_{n}\left(\frac{\partial}{\partial t} \alpha_{\xi C m}^{s}\left(t_{3}\right)\right)_{n}}=0 \\
& \overline{\left(\frac{\partial}{\partial t} \alpha_{\mu A k}^{s}\left(t_{1}\right)\right)_{n}\left(\frac{\partial}{\partial t} \alpha_{\nu B l}^{s}\left(t_{2}\right)\right)_{n}\left(\frac{\partial}{\partial t} \alpha_{\xi C m}^{s}\left(t_{3}\right)\right)_{n}\left(\frac{\partial}{\partial t} \alpha_{\lambda D p}^{s}\left(t_{4}\right)\right)_{n}} \\
= & \delta\left(t_{1}-t_{2}\right) \delta\left(t_{3}-t_{4}\right) \overline{E_{A k B l}^{\mu \nu}\left(t_{1,2}\right) E_{C m D p}^{\xi \lambda}\left(t_{3,4}\right)} \\
+ & \delta\left(t_{1}-t_{3}\right) \delta\left(t_{2}-t_{4}\right) \overline{\frac{E_{A k C m}^{\mu \xi}\left(t_{1,3}\right) E_{B l D p}^{\nu \lambda}\left(t_{2,4}\right)}{E_{A k D p}^{\mu \lambda}\left(t_{1,4}\right) E_{B l C m}^{\nu \xi}\left(t_{2,3}\right)}} \\
+ & \delta\left(t_{1}-t_{4}\right) \delta\left(t_{2}-t_{3}\right)
\end{aligned}
$$

Note that the phase noise terms are not themselves Gaussian-Markoff processes. 


\section{Theory - Quantum Field Treatment}

In this section the field operator and functional phase space approach is developed for the situation treated in Section 2 where the modes are divided into condensate and non-condensate modes. In general, the phase space functional approach is based on representing quantum density operator by a quasidistribution functional in a phase space involving field functions which replace the bosonic field annihilation, creation operators. Functionals and key properties are summarised in Appendix 6. In this hybrid approach time dependent condensate and non-condensate field operators are involved and these are represented by time dependent condensate and non-condensate field functions. The distribution functional will be of the Wigner type for the condensate field and of the positive $\mathrm{P}$ type for the non-condensate field. As shown in Appendix 7 , this treatment is equivalent to the treatment in Section 2 based on quasi-distribution functions of phase space variables that replace the annihilation, creation operators for the separate modes. Details of derivations are contained in Appendices 9 and 10 ,

\subsection{Condensate and Non-Condensate Field Operators}

Field operators $\hat{\Psi}_{C}(x)$ and $\hat{\Psi}_{N C}(x)$ for condensate and non-condensate modes respectively may also be defined in which the sums in (8) are over restricted sets of modes [14. The condensate and non-condensate field operators are

$$
\begin{aligned}
\hat{\Psi}_{C}(x, t) & =\sum_{k \in C} \widehat{a}_{k}(t) \phi_{k}(x, t), & \hat{\Psi}_{C}^{\dagger}(x, t)=\sum_{k \in C} \widehat{a}_{k}^{\dagger}(t) \phi_{k}^{*}(x, t) \\
\hat{\Psi}_{N C}(x, t) & =\sum_{k \in N C} \widehat{a}_{k}(t) \phi_{k}(x, t), & \hat{\Psi}_{N C}^{\dagger}(x, t)=\sum_{k \in N C} \widehat{a}_{k}^{\dagger}(t) \phi_{k}^{*}(x, t)
\end{aligned}
$$

where the mode sums are restricted to be only over condensate or non-condensate modes. Their sum gives the total field operators

$$
\hat{\Psi}(x)=\hat{\Psi}_{C}(x, t)+\hat{\Psi}_{N C}(x, t) \quad \hat{\Psi}^{\dagger}(x)=\hat{\Psi}_{C}^{\dagger}(x, t)+\hat{\Psi}_{N C}^{\dagger}(x, t)
$$

which satisfy (8).

These operators satisfy commutation rules involving restricted delta functions (see (6)).

$$
\begin{aligned}
{\left[\hat{\Psi}_{C}(x, t), \hat{\Psi}_{C}^{\dagger}(y, t)\right] } & =\delta_{C}(x, y) \quad\left[\hat{\Psi}_{N C}(x, t), \hat{\Psi}_{N C}^{\dagger}(y, t)\right]=\delta_{N C}(x, y) \\
{\left[\hat{\Psi}_{C}(x, t), \hat{\Psi}_{N C}^{\dagger}(y, t)\right] } & =\left[\hat{\Psi}_{N C}(x, t), \hat{\Psi}_{C}^{\dagger}(y, t)\right]=0 \\
\delta_{C}(x, y)+\delta_{C}(x, y) & =\delta(x-y)
\end{aligned}
$$

and condensate and non-condensate operators commute. In terms of projectors

$$
\hat{\Psi}_{C}(x)=\mathcal{P}_{x}^{C}[\hat{\Psi}(x)] \quad \hat{\Psi}_{N C}(x)=\mathcal{P}_{x}^{N C}[\hat{\Psi}(x)]
$$

We note that the condensate and non-condensate field operators are time dependent - it is only their sum that is time independent, as shown in (15). The 
feature that the separate field operators are time dependent requires significant modifications to the standard derivations of the FFPE and Ito stochastic field equations. We find that

$$
\begin{aligned}
\frac{\partial}{\partial t} \hat{\Psi}_{C}(x, t) & =\sum_{k \in C} \sum_{l \in N C} C_{k l} \widehat{a}_{l}(t) \phi_{k}(x, t)+\sum_{l \in C} \sum_{k \in N C} \widehat{a}_{l}(t) C_{l k}^{*} \phi_{k}(x, t) \\
& \neq 0
\end{aligned}
$$

because now there are pairs of $k, l$ such as $k \epsilon C, l \in N C$ for which the cancellation of $C_{k l}$ via the required $C_{l k}^{*}$ using (13) cannot occur, since for the latter $l \epsilon C, k \epsilon N C$. The other three condensate and non-condensate field operators are also time dependent.

\subsection{Condensate, Non-Condensate and Total Fields}

The total field operators $\hat{\Psi}(x), \hat{\Psi}^{\dagger}(x)$ in Eqs. (8) are represented by total field functions, which are defined by equations analogous to (8) for the field operators

$$
\psi(x, t)=\sum_{k} \alpha_{k}(t) \phi_{k}(x, t)=\psi(x) \quad \psi^{+}(x, t)=\sum_{k} \alpha_{k}^{+}(t) \phi_{k}^{*}(x, t)=\psi^{+}(x)
$$

where $\alpha_{k}(t), \alpha_{k}^{+}(t)$ are time dependent mode phase variables that satisfy Eq. (37). Using Eq.(14) and $C_{k l}+C_{l k}^{*}=0$ we see that the total field functions are actually time independent.

$$
\frac{\partial}{\partial t} \psi(x, t)=0 \quad \frac{\partial}{\partial t} \psi^{+}(x, t)=0
$$

This result depended on all of the $\phi_{k}(x, t)$ or $\phi_{k}^{*}(x, t)$ being involved in the field functions, since expanding $\frac{\partial}{\partial t} \phi_{k}(x, t)$ or $\frac{\partial}{\partial t} \phi_{k}^{*}(x, t)$ involves all modes. Modifications are needed when restricted sets of modes are involved for condensate and non-condensate field functions.

However, in the hybrid approach where condensate and non-condensate modes are treated differently, the phase space functional approach is based on representing quantum density operator by a quasi-distribution functional in a phase space involving four field functions which replace the bosonic field annihilation, creation operators $\hat{\Psi}_{C}(x, t), \hat{\Psi}_{C}^{\dagger}(x, t), \hat{\Psi}_{N C}(x, t), \hat{\Psi}_{N C}^{\dagger}(x, t)$. The condensate and non-condensate field functions are defined by equations analogous to Eq. (93) for the field operators

$$
\begin{aligned}
\psi_{C}(x, t) & =\sum_{k \in C} \alpha_{k}(t) \phi_{k}(x, t) & \psi_{C}^{+}(x, t)=\sum_{k \in C} \alpha_{k}^{+}(t) \phi_{k}^{*}(x, t) \\
\psi_{N C}(x, t) & =\sum_{k \in N C} \alpha_{k}(t) \phi_{k}(x, t) & \psi_{N C}^{+}(x, t)=\sum_{k \in N C} \alpha_{k}^{+}(t) \phi_{k}^{*}(x, t)(101)
\end{aligned}
$$

where $\alpha_{k}(t), \alpha_{k}^{+}(t)$ are time dependent mode phase variables that satisfy Eq. (37). In this hybrid case the field functions are time dependent. Similar to Eq. 
(98) the time dependences of the field functions are

$$
\begin{aligned}
\frac{\partial}{\partial t} \psi_{C}(x, t) & =\sum_{k \in C} \sum_{l \in N C} C_{k l} \alpha_{l}(t) \phi_{k}(x, t)+\sum_{l \in C} \sum_{k \in N C} \alpha_{l}(t) C_{l k}^{*} \phi_{k}(x, t) \\
\frac{\partial}{\partial t} \psi_{C}^{+}(x, t) & =\sum_{k \in C} \sum_{l \in N C} C_{k l}^{*} \alpha_{l}^{+}(t) \phi_{k}^{*}(x, t)+\sum_{l \in C} \sum_{k \in N C} \alpha_{l}^{+}(t) C_{l k} \phi_{k}^{*}(x, t) \\
\frac{\partial}{\partial t} \psi_{N C}(x, t) & =\sum_{k \in N C} \sum_{l \epsilon C} C_{k l} \alpha_{l}(t) \phi_{k}(x, t)+\sum_{l \in N C} \sum_{k \in C} \alpha_{l}(t) C_{l k}^{*} \phi_{k}(x, t) \\
\frac{\partial}{\partial t} \psi_{N C}^{+}(x, t) & =\sum_{k \in N C} \sum_{l \in C} C_{k l}^{*} \alpha_{l}^{+}(t) \phi_{k}^{*}(x, t)+\sum_{l \in N C} \sum_{k \in C} \alpha_{l}^{+}(t) C_{l k} \phi_{k}^{*}(x, t)
\end{aligned}
$$

However, in this case there is incomplete cancellation because $C_{k l}$ and $C_{l k}^{*}$ involve different pairs of $k, l$ so these derivatives are non-zero.

\subsection{Characteristic and Distribution Functionals}

The field theory phase space approach involves introducing characteristic functionals that can be used to specify all the quantum field correlation functions for a given density operator.We first define four time dependent characteristic field functions via

$$
\begin{aligned}
\Xi_{C}(x, t) & =\sum_{k \in C} \xi_{k}(t) \phi_{k}(x, t) & \Xi_{C}^{+}(x, t)=\sum_{k \in C} \xi_{k}^{+}(t) \phi_{k}^{*}(x, t) \\
\Xi_{N C}(x, t) & =\sum_{k \in N C} \xi_{k}(t) \phi_{k}(x, t) & \Xi_{N C}^{+}(x, t)=\sum_{k \in N C} \xi_{k}^{+}(t) \phi_{k}^{*}(x, t)(10
\end{aligned}
$$

with the same time dependent c-numbers $\xi_{k}(t), \xi_{k}^{+}(t)$ as before. These satisfy Eq.(41). These sum to total characteristic field functions

$$
\Xi^{+}=\Xi_{C}^{+}+\Xi_{N C}^{+} \quad \Xi^{-}=\Xi_{C}^{-}+\Xi_{N C}^{-}
$$

The hybrid characteristic functional $\chi\left[\Xi_{C}, \Xi_{C}^{+}, \Xi_{N C}, \Xi_{N C}^{+}\right]$is defined via

$$
\begin{aligned}
\chi\left[\Xi_{C}, \Xi_{C}^{+}, \Xi_{N C}, \Xi_{N C}^{+}\right] & =\operatorname{Tr}\left(\hat{\Omega}^{W}\left[\Xi_{C}, \Xi_{C}^{+}\right] \hat{\Omega}^{+}\left[\Xi_{N C}^{+}\right] \hat{\rho} \hat{\Omega}^{-}\left[\Xi_{N C}\right]\right) \\
\hat{\Omega}^{+}\left[\Xi_{N C}^{+}\right] & =\exp i \int d x \hat{\Psi}_{N C}(x, t) \Xi_{N C}^{+}(x, t) \\
\hat{\Omega}^{-}\left[\Xi_{N C}\right] & =\exp i \int d x \Xi_{N C}(x, t) \hat{\Psi}_{N C}^{\dagger}(x, t) \\
\hat{\Omega}^{W}\left[\Xi_{C}, \Xi_{C}^{+}\right] & =\exp i \int d x\left(\hat{\Psi}_{C}(x, t) \Xi_{C}^{+}(x, t)+\Xi_{C}(x, t) \hat{\Psi}_{C}^{\dagger}(x, t)\right)
\end{aligned}
$$


By applying the Baker-Hausdorff theorem we find that

$$
\begin{aligned}
\hat{\Omega}^{W}\left[\Xi_{C}, \Xi_{C}^{+}\right] & =\hat{\Omega}^{-}\left[\Xi_{C}\right] \hat{\Omega}^{+}\left[\Xi_{C}^{+}\right] \exp \left(-\frac{1}{2} \int d x \Xi_{C}(x, t) \Xi_{C}^{+}(x, t)\right) \\
\hat{\Omega}^{+}\left[\Xi_{C}^{+}\right] & =\exp i \int d x \hat{\Psi}_{C}(x, t) \Xi_{C}^{+}(x, t) \\
\hat{\Omega}^{-}\left[\Xi_{C}\right] & =\exp i \int d x \Xi_{C}(x, t) \hat{\Psi}_{C}^{\dagger}(x, t)
\end{aligned}
$$

we find that the overall characteristic functional is given by

$\chi\left[\Xi_{C}, \Xi_{C}^{+}, \Xi_{N C}, \Xi_{N C}^{+}\right]=\exp \left\{-\frac{1}{2} \int d x \Xi_{C}(x, t) \Xi_{C}^{+}(x, t)\right\} \chi_{P+}\left[\Xi_{C}, \Xi_{C}^{+}, \Xi_{N C}, \Xi_{N C}^{+}\right]$

where

$$
\begin{aligned}
& \chi_{P+}\left[\Xi_{C}, \Xi_{C}^{+}, \Xi_{N C}, \Xi_{N C}^{+}\right] \\
= & \operatorname{Tr}\left(\left\{\exp i \int d x\left(\hat{\Psi}_{C}(x, t) \Xi_{C}^{+}(x, t)+\hat{\Psi}_{N C}(x, t) \Xi_{N C}^{+}(x, t)\right)\right\} \hat{\rho}\right. \\
& \times\left\{\exp i \int d x\left(\Xi_{C}(x, t) \hat{\Psi}_{C}^{\dagger}(x, t)+\Xi_{N C}(x, t) \hat{\Psi}_{N C}^{\dagger}(x, t)\right)\right\} \\
= & \operatorname{Tr}\left(\left\{\exp i \int d x\left(\hat{\Psi}(x) \Xi^{+}(x)\right)\right\} \hat{\rho}\left\{\exp i \int d x\left(\Xi(x) \hat{\Psi}^{\dagger}(x)\right)\right\}\right.
\end{aligned}
$$

is an auxiliary characteristic functional. Similar to the separate mode situation, this characteristic functional is that applying if both the condensate and non-condensate fields were treated via a normally ordered characteristic functional. Here $\Xi(x), \Xi^{+}(x)$ are as in Eq. (104) for the total field and $\hat{\Psi}(x), \hat{\Psi}^{\dagger}(x)$ are the total field operators as in Eq.(8), both involving all the modes and both of which are time independent. Details in deriving (108) are set out in Appendix 9. Apart from the density operator the only quantity in the expression (107) for the characteristic functional that is time dependent is the factor $\exp \left\{-\frac{1}{2} \int d x \Xi_{C}(x, t) \Xi^{+}(x, t)\right\}$, and this simplifies the derivation of the functional Fokker-Planck equation. Eq. (107) relates the actual characteristic functional to that for the case where all modes are treated via a normally ordered characteristic functional.

By comparing the expression (107) for the characteristic functional with (30) for the hybrid characteristic function it follows that the characteristic functionals and characteristic functions are equivalent. They both contain the same information - in one case as a functional of the fields $\Xi_{C}, \Xi_{C}^{+}, \Xi_{N C}, \Xi_{N C}^{+}$in the other as a function of all the expansion coefficients $\left\{\xi_{k}(t), \xi_{k}^{+}(t)\right\}$ for both condensate and non-condensate modes.

$$
\chi\left[\Xi_{C}, \Xi_{C}^{+}, \Xi_{N C}, \Xi_{N C}^{+}\right] \equiv \chi\left(\xi, \xi^{+}\right)
$$

The phase space integral that relates the distribution function to the characteristic function can be expressed as a phase space functional integral in which 
the distribution function $P\left(\alpha, \alpha^{+}, \alpha^{*}, \alpha^{+*}\right)$ is replaced by equivalent distribution functionals

$$
P\left[\underset{\psi}{\rightarrow}, \stackrel{\psi}{\rightarrow}^{*}\right]=P\left(\alpha, \alpha^{+}, \alpha^{*}, \alpha^{+*}\right)
$$

where for short we introduce the notation

$$
\stackrel{\psi}{\rightarrow} \equiv\left\{\psi_{C}, \psi_{C}^{+}, \psi_{N C}, \psi_{N C}^{+}\right\} \quad \underline{\psi}^{*} \equiv\left\{\psi_{C}^{*}, \psi_{C}^{+*}, \psi_{N C}^{*}, \psi_{N C}^{+*}\right\}
$$

The equivalence of functional and phase space integration based on separate modes can be established using the methods of functional calculus (see Appendix [6. see Eq.(200) ). From Eq.(40) the phase space integration is time independent even though the phase variables depend on time. Thus

$$
\begin{aligned}
& \iiint \int D^{2} \psi_{C} D^{2} \psi_{C}^{+} D^{2} \psi_{N C} D^{2} \psi_{N C}^{+} \\
= & \iiint \int d^{2} \alpha_{C} d^{2} \alpha_{C}^{+} d^{2} \alpha_{N C} d^{2} \alpha_{N C}^{+} \\
= & \iiint \int d^{2} \alpha_{C}(0) d^{2} \alpha_{C}^{+}(0) d^{2} \alpha_{N C}(0) d^{2} \alpha_{N C}^{+}(0)
\end{aligned}
$$

indicating that functional integration is also essentially non time dependent. Note that all modes are involved as are the four fields $\psi_{C}, \psi_{C}^{+}, \psi_{N C}, \psi_{N C}^{+}$which are equivalent to the set of all $\alpha_{k}, \alpha_{k}^{+}$. Note that the functional integration $\int D^{2} \psi_{C}$ and $\int D^{2} \psi_{C}^{+}$involve a space grid with the same number of intervals as condensate modes. ).

The characteristic function is still related to the quasi-distribution function $P\left(\alpha, \alpha^{+}, \alpha^{*}, \alpha^{+*}\right)$ via Eq.(31) so writing this relationship in terms of functional integration we have an equivalent relation between the hybrid characteristic and distribution functionals

$$
\begin{aligned}
& \chi\left[\Xi_{C}, \Xi_{C}^{+}, \Xi_{N C}, \Xi_{N C}^{+}\right] \\
= & \iiint \int D^{2} \psi_{C} D^{2} \psi_{C}^{+} D^{2} \psi_{N C} D^{2} \psi_{N C}^{+} \\
& \times \exp \left(i \int d x\left(\psi_{C}(x, t) \Xi_{C}^{+}(x, t)+\Xi_{C}(x, t) \psi_{C}^{+}(x, t)\right)\right) \\
& \times \exp \left(i \int d x \psi_{N C}(x, t) \Xi_{N C}^{+}(x, t)\right) P\left[\underline{\psi}, \underline{\psi}^{*}\right] \exp \left(i \int d x \Xi_{N C}(x, t) \psi_{N C}^{+}(x, t)\right) \\
= & \iiint \int D^{2} \psi_{C} D^{2} \psi_{C}^{+} D^{2} \psi_{N C} D^{2} \psi_{N C}^{+} \\
& \times \exp \left(i \int d x\left(\psi_{C}(x, t) \Xi_{C}^{+}(x, t)+\psi_{N C}(x, t) \Xi_{N C}^{+}(x, t)+\Xi_{C}(x, t) \psi_{C}^{+}(x, t)+\Xi_{N C}(x, t) \psi_{N C}^{+}(x, t)\right)\right) \\
& \times P\left[\psi_{C}, \psi_{C}^{+}, \psi_{N C}, \psi_{N C}^{+}, \psi_{C}^{*}, \psi_{C}^{+*}, \psi_{N C}^{*}, \psi_{N C}^{+*}\right]
\end{aligned}
$$

where in the second line all the exponentials have been combined. The quasidistribution functional is of the Wigner type for the condensate fields and the positive $\mathrm{P}$ type for the non-condensate fields, as in [14. 
These results are the same as those for the case of time independent phase space variables. We note that

$$
\begin{aligned}
\int d x\left(\psi_{C}(x, t) \Xi_{C}^{+}(x, t)+\psi_{N C}(x, t) \Xi_{N C}^{+}(x, t)\right) & =\sum_{k \in C, N C} \alpha_{k} \xi_{k}^{+} \\
\int d x\left(\Xi_{C}(x, t) \psi_{C}^{+}(x, t)+\Xi_{N C}(x, t) \psi_{N C}^{+}(x, t)\right) & =\sum_{k \in C, N C} \xi_{k} \alpha_{k}^{+}
\end{aligned}
$$

which are time independent as before. Other details are given in Appendix 9 .

\subsubsection{Notation Change}

As in Section 2 we introduce the notation where we list the modes as $\phi_{A k}^{\mu}(x, t)$ with $\mu=-,+$ and $A=C, N C$. Thus $\phi_{A k}^{-}(x, t)=\phi_{A k}(x, t), \phi_{A k}^{+}(x, t)=\phi_{A k}^{*}(x, t)$. The condensate and non-condensate fields are listed as $\psi_{A}^{\mu}(x, t)$ and $\Xi_{A}^{\mu}(x, t)$, with $\psi_{C}^{-}(x, t)=\psi_{C}(x, t), \psi_{C}^{+}(x, t)=\psi_{C}^{+}(x, t), \psi_{N C}^{-}(x, t)=\psi_{N C}(x, t)$ and $\psi_{N C}^{+}(x, t)=$ $\psi_{N C}^{+}(x, t)$. The total fields are $\psi^{\mu}(x, t)=\psi_{C}^{\mu}(x, t)+\psi_{N C}^{\mu}(x, t)$ and $\Xi^{\mu}(x, t)=$ $\Xi_{C}^{\mu}(x, t)+\Xi_{N C}^{\mu}(x, t)$. The coupling constants are listed as $C_{A k, B l}^{\mu}$ as in Eq.(58). Writing $\underset{\rightarrow}{\rightarrow} . \equiv\left\{\psi_{C}, \psi_{C}^{+}, \psi_{N C}, \psi_{N C}^{+}\right\}$and $\underline{\psi}^{*} . \equiv\left\{\psi_{C}^{*}, \psi_{C}^{+*}, \psi_{N C}^{*}, \psi_{N C}^{+*}\right\}$ then we have

$\iiint \int D^{2} \psi_{C} D^{2} \psi_{C}^{+} D^{2} \psi_{N C} D^{2} \psi_{N C}^{+}$will be written as $\int D^{2} \underline{\psi}$. In the new notation with phase space variables now $\alpha_{\mu A k}(t)$ and the field functions are

$$
\psi_{A}^{\mu}(x, t)=\sum_{k} \alpha_{\mu A k}(t) \phi_{A k}^{\mu}(x, t)
$$

Inverting Eq.(115) gives

$$
\alpha_{\mu A k}=\int d x \phi_{A k}^{-\mu}(x, t) \psi_{A}^{\mu}(x, t)
$$

Also from Eqs. (56), (60) and (116) we see that with

$$
\begin{aligned}
& \frac{\partial}{\partial t} \psi_{A}^{\mu}(x, t) \\
= & \int d y \sum_{k B l} \phi_{A k}^{-\mu}(y, t) C_{A k B l}^{-\mu} \phi_{B l}^{\mu}(x, t) \psi_{A}^{\mu}(y, t)+\int d y \sum_{k B l} \phi_{A k}^{\mu}(x, t) C_{A k B l}^{\mu} \phi_{B l}^{-\mu}(y, t) \psi_{B}^{\mu}(y, t) \\
= & -\int d y \sum_{\substack{k B l \\
B \neq A}} \phi_{B l}^{\mu}(x, t) C_{B l A k}^{\mu} \phi_{A k}^{-\mu}(y, t) \psi_{A}^{\mu}(y, t)+\int d y \sum_{\substack{k B l \\
B \neq A}} \phi_{A k}^{\mu}(x, t) C_{A k B l}^{\mu} \phi_{B l}^{-\mu}(y, t) \psi_{B}^{\mu}(y, t)
\end{aligned}
$$

showing that the time derivative of the field function $(C, N C)$ is a functional of both the original field function $(C, N C)$ and the other field function $(N C, C)$. Note that the $B=A$ terms cancel because of $C_{A k A l}^{\mu}+C_{A l A k}^{-\mu}=0$. 


\subsection{Quantum Correlation Functions}

The quantum correlation functions for the field operators can be obtained from the characteristic functionals via taking functional derivatives with respect to $\Xi_{C}, \Xi_{C}^{+}, \Xi_{N C}, \Xi_{N C}^{+}$and then setting these quantities to be zero. The result gives the quantum correlation functions as phase space functional integrals involving the distribution functionals. Thus the quantum correlation function is

$$
\begin{aligned}
& G\left(r_{1} \cdots r_{p} ; s_{q} \cdots s_{1} ; u_{1} \cdots u_{r} ; v_{s} \cdots v_{1}\right) \\
= & \left\langle\left\{\hat{\Psi}_{C}\left(r_{1}\right)^{\dagger} \cdots \hat{\Psi}_{C}\left(r_{p}\right)^{\dagger} \hat{\Psi}_{C}\left(s_{q}\right) \cdots \hat{\Psi}_{C}\left(s_{1}\right)\right\} \hat{\Psi}_{N C}\left(u_{1}\right)^{\dagger} \cdots \hat{\Psi}_{N C}\left(u_{r}\right)^{\dagger} \hat{\Psi}_{N C}\left(v_{s}\right) \cdots \hat{\Psi}_{N C}\left(v_{1}\right)\right\rangle \\
= & \iiint \int D^{2} \psi_{C} D^{2} \psi_{C}^{+} D^{2} \psi_{N C} D^{2} \psi_{N C}^{+} \\
& \times \psi_{C}^{+}\left(r_{1}\right) \cdots \psi_{C}^{+}\left(r_{p}\right) \psi_{C}\left(s_{q}\right) \cdots \psi_{C}\left(s_{1}\right) \psi_{N C}^{+}\left(u_{1}\right) \cdots \psi_{N C}^{+}\left(u_{r}\right) \psi_{N C}\left(v_{s}\right) \cdots \psi_{N C}\left(v_{1}\right) P\left[\underline{\psi}, \stackrel{\psi}{\rightarrow}_{\rightarrow}^{*}\right]
\end{aligned}
$$

For simplicity the $t$ dependences of the condensate and non-condensate field operators and field functions have been left out.

\subsection{Functional Fokker-Planck Equations}

To derive the functional Fokker-Planck equation we first differentiate the expression in Eq. (107) for the characteristic functional with respect to $t$ and second do the same to the expression (113) involving the distribution functional.

Differentiating the characteristic functional in its first form (107) we have

$$
\begin{aligned}
& \frac{\partial}{\partial t} \chi\left[\Xi_{C}, \Xi_{C}^{+}, \Xi_{N C}, \Xi_{N C}^{+}\right] \\
= & \exp \left\{-\frac{1}{2} \int d x \Xi_{C}(x, t) \Xi_{C}^{+}(x, t)\right\} \\
& \times \operatorname{Tr}\left(\left\{\exp i \int d x\left(\hat{\Psi}(x) \Xi^{+}(x)\right)\right\}\left(\frac{\partial}{\partial t} \hat{\rho}\right)\left\{\exp i \int d x\left(\Xi(x) \hat{\Psi}^{\dagger}(x)\right)\right\}\right) \\
& +\left(\frac{\partial}{\partial t} \exp \left\{-\frac{1}{2} \int d x \Xi_{C}(x, t) \Xi_{C}^{+}(x, t)\right\}\right) \\
& \times \operatorname{Tr}\left(\left\{\exp i \int d x\left(\hat{\Psi}(x) \Xi^{+}(x)\right)\right\} \hat{\rho}\left\{\exp i \int d x\left(\Xi(x) \hat{\Psi}^{\dagger}(x)\right)\right\}\right)
\end{aligned}
$$

This result gives the time derivative of the characteristic functional as the sum of two terms. The first term in (119) only involves the time derivative of the density operator since from Eq. (215) and (42) the integrals involving $\Xi(x)$ and $\Xi^{+}(x)$ are time independent. This term equals the characteristic functional that would apply if $\hat{\rho}$ is replaced by $\frac{\partial}{\partial t} \hat{\rho}$. The first term produces the standard terms in the functional Fokker-Planck equation. The second term depends on the time dependence of the $\int d x \Xi_{C}(x, t) \Xi_{C}^{+}(x, t)$ factor due to the time dependent 
modes and is equal to the characteristic functional multiplied by functionals of the condensate and non-condensate characteristic fields. The second term leads to additional diffusion terms in the functional Fokker-Planck equation.

For the second term in $\frac{\partial}{\partial t} \chi\left[\Xi_{C}, \Xi_{C}^{+}, \Xi_{N C}, \Xi_{N C}^{+}\right]$the quantity involving the time derivative is

$$
\begin{aligned}
& \frac{\partial}{\partial t} \exp \left\{-\frac{1}{2} \int d x \Xi_{C}(x, t) \Xi_{C}^{+}(x, t)\right\} \\
= & \left\{-\frac{1}{2} \frac{\partial}{\partial t} \int d x \Xi_{C}(x, t) \Xi_{C}^{+}(x, t)\right\} \times \exp \left\{-\frac{1}{2} \int d x \Xi_{C}(x, t) \Xi_{C}^{+}(x, t)\right\}
\end{aligned}
$$

and using

$$
\begin{aligned}
& -\frac{1}{2} \frac{\partial}{\partial t} \int d x \Xi_{C}(x, t) \Xi_{C}^{+}(x, t) \\
= & \frac{1}{2}\left\{\iint d x d y\left\{\sum_{k \in C} \sum_{l \in N C} \phi_{l}^{*}(x, t) C_{k l}(t) \phi_{k}(y, t)\right\}\left(i \Xi_{N C}(x, t)\right)\left(i \Xi_{C}^{+}(y, t)\right)\right\} \\
+ & \frac{1}{2}\left\{\iint d x d y\left\{\sum_{l \in C} \sum_{k \in N C} \phi_{l}^{*}(x, t) C_{l k}^{*}(t) \phi_{k}(y, t)\right\}\left(i \Xi_{C}(x, t)\right)\left(i \Xi_{N C}^{+}(y, t)\right)\right\}
\end{aligned}
$$

we find that the second term is given as the sum of two contributions as

$$
\begin{aligned}
& \left(-\frac{1}{2} \frac{\partial}{\partial t} \int d x \Xi_{C}(x, t) \Xi_{C}^{+}(x, t)\right) \chi\left[\Xi_{C}, \Xi_{C}^{+}, \Xi_{N C}, \Xi_{N C}^{+}\right] \\
= & \int D^{2} \underline{\psi} \exp \left(i \int d x \sum_{\mu} \psi^{\mu}(x, t) \Xi^{-\mu}(x, t)\right) \\
& \times \iint d x d y\left\{\frac{1}{2} \sum_{k \epsilon C} \sum_{l \epsilon N C} \phi_{N C l}^{+}(x, t) C_{C k N C l}^{-}(t) \phi_{C k}^{-}(y, t) \frac{\delta}{\delta \psi_{N C}^{+}(x, t)} \frac{\delta}{\delta \psi_{C}^{-}(y, t)} P_{\rightarrow}\left[\underline{\psi}^{*}\right]\right\} \\
& +\int D^{2} \underline{\psi} \exp \left(i \int d x \sum_{\mu} \psi^{\mu}(x, t) \Xi^{-\mu}(x, t)\right) \\
& \times \iint d x d y\left\{\frac{1}{2} \sum_{k \in N C} \sum_{l \epsilon C} \phi_{C l}^{+}(x, t) C_{C l N C k}^{+}(t) \phi_{N C k}^{-}(y, t) \frac{\delta}{\delta \psi_{C}^{+}(x, t)} \frac{\delta}{\delta \psi_{N C}^{-}(y, t)} P\left[\underline{\psi}, \underline{\left.\psi^{*}\right]}\right\}\right.
\end{aligned}
$$

in terms of the new notation. In obtaining this result the exponential factor in Eq. (120) is recombined via (107) with the normally ordered characteristic functional $\operatorname{Tr}\left(\left\{\exp i \int d x\left(\hat{\Psi}(x) \Xi^{+}(x)\right)\right\} \hat{\rho}\left\{\exp i \int d x\left(\Xi(x) \hat{\Psi}^{\dagger}(x)\right)\right\}\right)$ to produce the original characteristic functional $\chi\left[\Xi_{C}, \Xi_{C}^{+}, \Xi_{N C}, \Xi_{N C}^{+}\right]$. Writing the characteristic functional in the form in Eq. (113) and noting that multiplication by 
$\left(i \Xi_{N C}(x, t)\right)\left(i \Xi_{C}^{+}(y, t)\right)$ etc. can be replaced by functional differentiations such as $\frac{\delta}{\delta \psi_{N C}^{+}(x, t)} \frac{\delta}{\delta \psi_{C}(y, t)}$ result (122) follows after applying functional integration by parts twice. Details for the derivation of (121) are set out in Appendix 9 .

For the first term in $\frac{\partial}{\partial t} \chi\left[\Xi_{C}, \Xi_{C}^{+}, \Xi_{N C}, \Xi_{N C}^{+}\right]$we use the standard results for the correspondence rules associated with replacing the density operator by its product with the condensate and non-condensate field annihilation, creation operators $\hat{\Psi}_{C}(x, t), \hat{\Psi}_{C}^{\dagger}(x, t), \hat{\Psi}_{N C}(x, t), \hat{\Psi}_{N C}^{\dagger}(x, t)$ that occur when $\frac{\partial}{\partial t} \hat{\rho}$ is evaluated using the Liouville-von Neumann or master equation. For the noncondensate field operators we have

$$
\begin{array}{ll}
\hat{\rho} \Rightarrow \hat{\Psi}_{N C}(x, t) \hat{\rho} & \chi\left[\Xi_{C}, \Xi_{C}^{+}, \Xi_{N C}, \Xi_{N C}^{+}\right] \Rightarrow \frac{\delta}{\delta\left(i \Xi_{N C}^{+}(x, t)\right)} \chi \\
\hat{\rho} \Rightarrow \hat{\rho} \hat{\Psi}_{N C}(x, t) & \chi\left[\Xi_{C}, \Xi_{C}^{+}, \Xi_{N C}, \Xi_{N C}^{+}\right] \Rightarrow\left(\frac{\delta}{\delta\left(i \Xi_{N C}^{+}(x, t)\right)}+i \Xi_{N C}(x, t)\right) \chi \\
\hat{\rho} \Rightarrow \hat{\Psi}_{N C}^{\dagger}(x, t) \hat{\rho} & \chi\left[\Xi_{C}, \Xi_{C}^{+}, \Xi_{N C}, \Xi_{N C}^{+}\right] \Rightarrow\left(\frac{\delta}{\delta\left(i \Xi_{N C}(x, t)\right)}+i \Xi_{N C}^{+}(x, t)\right) \chi \\
\hat{\rho} \Rightarrow \hat{\rho} \hat{\Psi}_{N C}^{\dagger}(x, t) & \chi\left[\Xi_{C}, \Xi_{C}^{+}, \Xi_{N C}, \Xi_{N C}^{+}\right] \Rightarrow \frac{\delta}{\delta\left(i \Xi_{N C}(x, t)\right)} \chi
\end{array}
$$

For the condensate field operators we have

$$
\begin{aligned}
& \hat{\rho} \Rightarrow \hat{\Psi}_{C}(x, t) \hat{\rho} \quad \chi\left[\Xi_{C}, \Xi_{C}^{+}, \Xi_{N C}, \Xi_{N C}^{+}\right] \Rightarrow\left(\frac{\delta}{\delta\left(i \Xi_{C}^{+}(x, t)\right)}-\frac{1}{2} i \Xi_{C}(x, t)\right) \chi \\
& \hat{\rho} \Rightarrow \hat{\rho} \hat{\Psi}_{C}(x, t) \quad \chi\left[\Xi_{C}, \Xi_{C}^{+}, \Xi_{N C}, \Xi_{N C}^{+}\right] \Rightarrow\left(\frac{\delta}{\delta\left(i \Xi_{C}^{+}(x, t)\right)}+\frac{1}{2} i \Xi_{C}(x, t)\right) \chi \\
& \hat{\rho} \Rightarrow \hat{\Psi}_{C}^{\dagger}(x, t) \hat{\rho} \quad \chi\left[\Xi_{C}, \Xi_{C}^{+}, \Xi_{N C}, \Xi_{N C}^{+}\right] \Rightarrow\left(\frac{\delta}{\delta\left(i \Xi_{C}(x, t)\right)}+\frac{1}{2} i \Xi_{C}^{+}(x, t)\right) \chi \\
& \hat{\rho} \Rightarrow \hat{\rho} \hat{\Psi}_{C}^{\dagger}(x, t) \quad \chi\left[\Xi_{C}, \Xi_{C}^{+}, \Xi_{N C}, \Xi_{N C}^{+}\right] \Rightarrow\left(\frac{\delta}{\delta\left(i \Xi_{C}(x, t)\right)}-\frac{1}{2} i \Xi_{C}^{+}(x, t)\right) \chi
\end{aligned}
$$

From these results the corresponding changes to the distribution functionals can be deduced using (105) and (113). This involves functional integration by parts.

The correspondence rules for the non-condensate field operators are

$$
\begin{aligned}
& \hat{\rho} \Rightarrow \hat{\Psi}_{N C}(x, t) \hat{\rho} \quad P\left[\stackrel{\psi}{\rightarrow}, \stackrel{\psi}{*}^{*}\right] \Rightarrow \psi_{N C}(x, t) P \\
& \hat{\rho} \Rightarrow \hat{\rho} \hat{\Psi}_{N C}(x, t) \quad P\left[\underset{\rightarrow}{\rightarrow}, \psi_{\rightarrow}^{*}\right] \Rightarrow\left(-\frac{\delta}{\delta \psi_{N C}^{+}(x, t)}+\psi_{N C}(x, t)\right) P \\
& \hat{\rho} \Rightarrow \hat{\Psi}_{N C}^{\dagger}(x, t) \hat{\rho} \quad P\left[\underset{\psi}{\rightarrow}, \psi^{*}\right] \Rightarrow\left(=\frac{\delta}{\delta \psi_{N C}(x, t)}+\psi_{N C}^{+}(x, t)\right) P \\
& \hat{\rho} \Rightarrow \hat{\rho} \hat{\Psi}_{N C}^{\dagger}(x, t) \quad P\left[\stackrel{\psi}{\rightarrow}, \stackrel{\psi}{\rightarrow}^{*}\right] \Rightarrow \psi_{N C}^{+}(x, t) P
\end{aligned}
$$


The correspondence rules for the condensate field operators are:

$$
\begin{aligned}
& \hat{\rho} \Rightarrow \hat{\Psi}_{C}(x, t) \hat{\rho} \quad P\left[\underset{\psi}{\rightarrow}, \psi_{\rightarrow}^{*}\right] \Rightarrow\left(\psi_{C}(x, t)+\frac{1}{2} \frac{\delta}{\delta \psi_{C}^{+}(x, t)}\right) P \\
& \hat{\rho} \Rightarrow \hat{\rho} \hat{\Psi}_{C}(x, t) \quad P\left[\underset{\psi}{\rightarrow}, \stackrel{\psi}{\rightarrow}^{*}\right] \Rightarrow\left(\psi_{C}(x, t)-\frac{1}{2} \frac{\delta}{\delta \psi_{C}^{+}(x, t)}\right) P \\
& \hat{\rho} \Rightarrow \hat{\Psi}_{C}^{\dagger}(x, t) \hat{\rho} \quad P\left[\underset{\psi}{\rightarrow}, \Psi^{*}\right] \Rightarrow\left(\psi_{C}^{+}(x, t)-\frac{1}{2} \frac{\delta}{\delta \psi_{C}(x, t)}\right) P \\
& \hat{\rho} \Rightarrow \hat{\rho} \hat{\Psi}_{C}^{\dagger}(x, t) \quad P\left[\underline{\psi}, \underline{\psi}_{\rightarrow}^{*}\right] \Rightarrow\left(\psi_{C}^{+}(x, t)+\frac{1}{2} \frac{\delta}{\delta \psi_{C}(x, t)}\right) P
\end{aligned}
$$

where the $\psi_{C}, \psi_{C}^{+}, \psi_{C}^{*}, \psi_{C}^{+*}, \psi_{N C}, \psi_{N C}^{+}, \psi_{N C}^{*}, \psi_{N C}^{+*}$ are regarded as eight independent complex fields. The equivalence of these correspondence rules to those applying for separate modes can be established using the methods of functional calculus (see Appendix 6, see Eq.(188) which relates ordinary and functional differentiation).

By applying the correspondence rules (125) or (126) in succession to the various products of the density operator with field annihilation, creation operators that arise from $\frac{\partial}{\partial t} \hat{\rho}$ we get for the first term in $\frac{\partial}{\partial t} \chi\left[\Xi_{C}, \Xi_{C}^{+}, \Xi_{N C}, \Xi_{N C}^{+}\right]$

$$
\begin{aligned}
& \chi\left[\Xi_{C}, \Xi_{C}^{+}, \Xi_{N C}, \Xi_{N C}^{+} ; \frac{\partial}{\partial t} \hat{\rho}\right] \\
= & \int D^{2} \underline{\psi} \exp \left(i \int d x \sum_{\mu} \psi^{\mu}(x, t) \Xi^{-\mu}(x, t)\right) \\
& \times\left\{-\sum_{\mu A} \int d x \frac{\delta}{\delta \psi_{A}^{\mu}(x)} A_{A}^{\mu}(x)+\frac{1}{2} \sum_{\mu A} \sum_{\nu B} \iint d x d y \frac{\delta}{\delta \psi_{A}^{\mu}(x)} \frac{\delta}{\delta \psi_{B}^{\nu}(y)} D_{A B}^{\mu \nu}(x, y)\right\} P\left[\underline{\psi}_{\rightarrow}, \stackrel{\psi}{\rightarrow}^{*}\right]
\end{aligned}
$$

in terms of the new notation, where $A_{A}^{\mu}(x), D_{A B}^{\mu \nu}(x, y)$ are the drift, diffusion terms associated with the standard functional Fokker-Planck equation. The integrals over $x, y$ arise because the Hamiltonian involves spatial integrals over the field operators. Specific forms for the drift, diffusion terms are given for the cases of a single or two mode single component BEC in [14. 
Differentiating the characteristic functional in its second form (113) we have

$$
\begin{aligned}
& \frac{\partial}{\partial t} \chi\left[\Xi_{C}, \Xi_{C}^{+}, \Xi_{N C}, \Xi_{N C}^{+} ; \hat{\rho}\right] \\
= & \iiint \int D^{2} \psi_{C} D^{2} \psi_{C}^{+} D^{2} \psi_{N C} D^{2} \psi_{N C}^{+} \\
& \times \exp \left(i \int d x\left(\psi_{C}(x, t) \Xi_{C}^{+}(x, t)+\psi_{N C}(x, t) \Xi_{N C}^{+}(x, t)+\Xi_{C}(x, t) \psi_{C}^{+}(x, t)+\Xi_{N C}(x, t) \psi_{N C}^{+}(x, t)\right)\right. \\
& \times \frac{\partial}{\partial t} P\left[\psi_{C}, \psi_{C}^{+}, \psi_{N C}, \psi_{N C}^{+}, \psi_{C}^{*}, \psi_{C}^{+*}, \psi_{N C}^{*}, \psi_{N C}^{+*}\right] \\
= & \left.\int D^{2} \underline{\psi} \exp \left(i \int d x \sum_{\mu} \psi^{\mu}(x, t) \Xi^{-\mu}(x, t)\right) \frac{\partial}{\partial t} \stackrel{P}{\rightarrow} \stackrel{\psi}{\rightarrow}, \stackrel{\psi}{\rightarrow}^{*}\right]
\end{aligned}
$$

in terms of the new notation. From Eq. 215) and (42) the integrals involving $\Xi_{C}(x, t), \Xi_{N C}(x, t), \Xi_{C}^{+}(x, t)$, and $\Xi_{N C}^{+}(x, t)$ are time independent, whilst the functional integration $\iiint \int D^{2} \psi_{C} D^{2} \psi_{C}^{+} D^{2} \psi_{N C} D^{2} \psi_{N C}^{+}$being the same as the phase space integration $\iiint \int d^{2} \alpha_{C} d^{2} \alpha_{C}^{+} d^{2} \alpha_{N C} d^{2} \alpha_{N C}^{+}=\iiint \int d^{2} \alpha_{C}(0) d^{2} \alpha_{C}^{+}(0) d^{2} \alpha_{N C}(0) d^{2} \alpha_{N C}^{+}(0)$ - is also time independent. It follows that the time derivative of the characteristic functional is now determined from the time derivative of the distribution functional.

Equating both sides of $\frac{\partial}{\partial t} \chi\left[\Xi_{C}, \Xi_{C}^{+}, \Xi_{N C}, \Xi_{N C}^{+}\right]$via Eqs. (122), (127) and (128) gives the functional Fokker-Planck equation for the hybrid distribution function. All the diffusion terms are combined, and there are new diffusion terms involving condensate fields paired with non-condensate fields arising due to the coupling coefficients. We have

$$
\begin{aligned}
& \frac{\partial}{\partial t} P\left[\underline{\psi}, \underline{\psi}^{*}\right] \\
= & \left\{-\sum_{\mu A} \int d x \frac{\delta}{\delta \psi_{A}^{\mu}(x, t)} A_{A}^{\mu}(x)+\frac{1}{2} \sum_{\mu A} \sum_{\nu B} \iint d x d y \frac{\delta}{\delta \psi_{A}^{\mu}(x, t)} \frac{\delta}{\delta \psi_{B}^{\nu}(y, t)} E_{A B}^{\mu \nu}(x, y)\right\} P\left[\underline{\psi}_{\rightarrow}, \underline{\psi^{*}}\right]
\end{aligned}
$$

where the new diffusion term is given by

$$
\begin{aligned}
E_{A B}^{\mu \nu}(x, y)= & D_{A B}^{\mu \nu}(x, y) \\
+ & \frac{1}{2}\left\{\delta_{A C} \delta_{B N C} \delta_{\mu-\nu}\left(\sum_{k} \sum_{l} \phi_{A k}^{\mu}(x, t) C_{A k B l}^{\mu}(t) \phi_{B l}^{\nu}(y, t)\right)\right\} \\
+ & \frac{1}{2}\left\{\delta_{B C} \delta_{A N C} \delta_{\nu-\mu}\left(\sum_{k} \sum_{l} \phi_{B l}^{\nu}(y, t) C_{B l A k}^{\nu}(t) \phi_{A k}^{\mu}(x, t)\right)\right\}
\end{aligned}
$$

Details are given in Appendix 9. Here the additional diffusion contribution has been rewritten in a manifestly symmetric form. The $\operatorname{drift} A_{A}^{\mu}(x)$ vector 
and the diffusion matrix $E_{A B}^{\mu \nu}(x, y)$ are functions of the $\psi_{A}^{\mu}$ and ultimately depend on $x$ or $x, y$. The diffusion matrix is symmetric $E_{A B}^{\mu \nu}(x, y)=E_{B A}^{\nu \mu}(y, x)$. Thus for the hybrid case the functional Fokker-Planck equation has the same drift vector $A_{A}^{\mu}(x)$ but a different diffusion matrix $E_{A B}^{\mu \nu}(x, y)$ to the diffusion matrix $D_{A B}^{\mu \nu}(x, y)$ that would be obtained from the characteristic functional by applying the standard correspondence rules to the $\frac{\partial}{\partial t} \hat{\rho}$ contribution. The additional terms involved the time dependent mode functions and the coupling coefficients. The functional Fokker-Planck equation can also be expressed in terms of real variables which involve the real and imaginary components of the phase variables $\psi_{\mu}$, but we will not do that here. In deriving the functional Fokker-Planck equation there are often terms involving third and higher order derivatives arising from the $\frac{\partial}{\partial t} \hat{\rho}$ contribution. These are usually discarded on the basis of being small due to scaling as higher powers of $1 / \sqrt{N}$.

The functional Fokker-Planck equation (129) for the distribution functional $P\left[\underline{\psi}, \underline{\psi}^{*}\right]$ must be equivalent to the ordinary Fokker-Planck equation Eq. (63) for the distribution function $P\left(\alpha, \alpha^{+}, \alpha^{*}, \alpha^{+*}\right)$. This is shown in Appendix 7 .

\subsection{Langevin (Ito) Stochastic Field Equations}

To derive the Ito stochastic field equations we follow a similar approach to Section 2.9, We consider a functional $F[\underline{\psi}]$ of the condensate and non-condensate fields and find conditions where the phase space functional average of the functional $F[\underline{\psi}]$ is the same as the stochastic average when the fields $\psi_{A}^{\mu}$ are replaced by stochastic fields $\psi_{A}^{\mu s}$.

\subsubsection{Phase Space Functional Average}

The phase space functional average is given by

$$
\left.\left\langle F[\underset{\rightarrow}{\rightarrow}\rangle_{t}=\int D^{2} \underset{\rightarrow}{\rightarrow} \underset{\rightarrow}{\rightarrow}\right] \underset{\rightarrow}{\rightarrow}, \underset{\rightarrow}{*}\right]
$$

For determining the quantum correlation function $G\left(r_{1} \cdots r_{p} ; s_{q} \cdots s_{1} ; ; u_{1} \cdots u_{r} ; v_{s} \cdots v_{1}\right)$ the functional is $F[\underline{\psi}]=\psi_{C}^{+}\left(r_{1}\right) \cdots \psi_{C}^{+}\left(r_{p}\right) \psi_{C}\left(s_{q}\right) \cdots \psi_{C}\left(s_{1}\right) \psi_{N C}^{+}\left(u_{1}\right) \cdots \psi_{N C}^{+}\left(u_{r}\right) \psi_{N C}\left(v_{s}\right) \cdots \psi_{N C}\left(v_{1}\right)$ - a product of functionals. In the phase space functional average both the distribution functional and $F[\underline{\psi}]$ are time dependent, because from Eq.(102) the

$\psi_{C}, \psi_{C}^{+}, \psi_{N C}, \psi_{N C}^{+}$depend on time. However from (112) the functional integration is not time dependent.

The time rate of change in the phase space functional average then consists of two contributions

$$
\begin{aligned}
& \frac{\partial}{\partial t}\langle F[\underline{\psi}]\rangle_{t}
\end{aligned}
$$

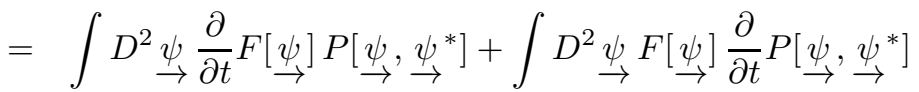


To evaluate the first term we first consider changes $\delta \psi_{A}^{\mu}(x, t)$ in $\psi_{A}^{\mu}(x, t)$ during an interval $\delta t$ that results in a change $\delta F[\underline{\psi}]$ to the functional $F[\underline{\psi}]$. From Eq.(182) we find that

$$
\frac{\partial}{\partial t} F[\underset{\psi}{\rightarrow}]=\sum_{\mu A} \int d x\left(\frac{\delta}{\delta \psi_{A}^{\mu}(x, t)} F[\underset{\psi}{\rightarrow}]\right) \frac{\partial \psi_{A}^{\mu}(x, t)}{\partial t}
$$

so substituting from Eq. (117) we get

$$
\begin{aligned}
& \frac{\partial}{\partial t} F[\underline{\psi}] \\
= & \sum_{\mu A} \int d x\left(\frac{\delta}{\delta \psi_{A}^{\mu}(x, t)} F[\underline{\psi}]\right)\left(\int d y \sum_{B}\left(\sum_{k l} \phi_{A k}^{\mu}(x, t) C_{A k B l}^{\mu} \phi_{B l}^{-\mu}(y, t)\right) \psi_{B}^{\mu}(y, t)\right) \\
& +\sum_{\mu A} \int d x\left(\frac{\delta}{\delta \psi_{A}^{\mu}(x, t)} F[\underline{\psi}]\right)\left(\int d y \sum_{B}\left(\sum_{k l} \phi_{B l}^{\mu}(x, t) C_{A k B l}^{-\mu} \phi_{A k}^{-\mu}(y, t)\right) \psi_{A}^{\mu}(y, t)\right)
\end{aligned}
$$

Hence the first term in (132) is given by

$$
\begin{aligned}
& \int D^{2} \underline{\psi} \rightarrow \frac{\partial}{\partial t} F[\underline{\psi}] P\left[\underline{\psi}, \underline{\psi}_{\rightarrow}^{*}\right] \\
= & \left\langle\sum_{\mu A} \int d x\left(\frac{\delta}{\delta \psi_{A}^{\mu}(x, t)} F[\underset{\psi}{\rightarrow}]\right)\left\{\int d y \sum_{B}\left(\sum_{k l} \phi_{A k}^{\mu}(x, t) C_{A k B l}^{\mu} \phi_{B l}^{-\mu}(y, t)\right) \psi_{B}^{\mu}(y, t)\right\}\right\rangle \\
& +\left\langle\sum_{\mu A} \int d x\left(\frac{\delta}{\delta \psi_{A}^{\mu}(x, t)} F[\underline{\rightarrow}]\right)\left\{\int d y \sum_{B}\left(\sum_{k l} \phi_{B l}^{\mu}(x, t) C_{A k B l}^{-\mu} \phi_{A k}^{-\mu}(y, t)\right) \psi_{A}^{\mu}(y, t)\right\}\right\rangle
\end{aligned}
$$

Details are given in Appendix 10.

Using the functional Fokker-Planck equation (129) we find after using integration by parts that the second term in (132) is

$$
\begin{aligned}
& \left.\int D^{2} \underset{\rightarrow}{\rightarrow} \underset{\rightarrow}{\rightarrow}\right] \frac{\partial}{\partial t} P\left[\underset{\rightarrow}{\rightarrow}, \stackrel{\psi}{*}^{*}\right] \\
& =\left\langle\sum_{\mu A} \int d x\left(\frac{\delta}{\delta \psi_{A}^{\mu}(x, t)} F[\underset{\psi}{\rightarrow}]\right) A_{A}^{\mu}(x)\right\rangle \\
& +\left\langle\frac{1}{2} \sum_{\mu A} \sum_{\nu B} \iint d x d y\left(\frac{\delta}{\delta \psi_{A}^{\mu}(x, t)} \frac{\delta}{\delta \psi_{B}^{\nu}(y, t)} F[\underset{\rightarrow}{\rightarrow}) E_{A B}^{\mu \nu}(x, y)\right\rangle\right.
\end{aligned}
$$

where functional integration by parts has been used. Details are given in Appendix 10, 
Overall we now have

$$
\begin{aligned}
& \frac{\partial}{\partial t}\left\langle F[\underset{\rightarrow}{ }\rangle_{t}\right. \\
= & \left\langle\sum_{\mu A} \int d x\left(\frac{\delta}{\delta \psi_{A}^{\mu}(x, t)} F[\underset{\rightarrow}{ }]\right)\left\{A_{A}^{\mu}(x)\right\}\right\rangle \\
& +\left\langle\sum_{\mu A} \int d x\left(\frac{\delta}{\delta \psi_{A}^{\mu}(x, t)} F[\underline{\rightarrow}]\right)\left\{\int d y \sum_{B}\left(\sum_{k l} \phi_{A k}^{\mu}(x, t) C_{A k B l}^{\mu} \phi_{B l}^{-\mu}(y, t)\right) \psi_{B}^{\mu}(y, t)\right\}\right\rangle \\
& +\left\langle\sum_{\mu A} \int d x\left(\frac{\delta}{\delta \psi_{A}^{\mu}(x, t)} F[\underline{\rightarrow}]\right)\left\{\int d y \sum_{B}\left(\sum_{k l} \phi_{B l}^{\mu}(x, t) C_{A k B l}^{-\mu} \phi_{A k}^{-\mu}(y, t)\right) \psi_{A}^{\mu}(y, t)\right\}\right\rangle \\
& +\left\langle\frac{1}{2} \sum_{\mu A} \sum_{\nu B} \iint d x d y\left(\frac{\delta}{\delta \psi_{A}^{\mu}(x, t)} \frac{\delta}{\delta \psi_{B}^{\nu}(y, t)} F\left[\underline{\psi}_{\rightarrow}\right]\right) E_{A B}^{\mu \nu}(x, y)\right\rangle
\end{aligned}
$$

This result can be compared to that for a function $F\left(\alpha, \alpha^{+}\right)$as set out in Section 2 where from Eq. (70)

$$
\begin{aligned}
& \frac{\partial}{\partial t}\left\langle F\left(\alpha, \alpha^{+}\right)\right\rangle_{t} \\
= & \left\langle\left\{\sum_{\mu A k}\left[\frac{\partial}{\partial \alpha_{\mu A k}} F\left(\alpha, \alpha^{+}\right)\right]\left[A_{A k}^{\mu}+\sum_{B l} C_{A k B l}^{\mu} \alpha_{\mu B l}\right]\right\}\right\rangle \\
& +\left\langle\left\{\frac{1}{2} \sum_{\mu A k} \sum_{\nu B l}\left[\frac{\partial}{\partial \alpha_{\mu A k}} \frac{\partial}{\partial \alpha_{\nu B l}} F\left(\alpha, \alpha^{+}\right)\right] E_{A k B l}^{\mu \nu}\right\}\right\rangle
\end{aligned}
$$

We see that the derivative of the functional phase space average is not the same form as that for the original phase space average involving separate modes, since a term corresponding to one of the first order functional derivative terms is missing. As a specific case consider the functional $F[\underline{\psi}]=\psi_{C}^{+}\left(r_{1}, t\right) \psi_{C}\left(s_{1}, t\right)$ that is involved in working out the symmetrically ordered correlation function $\left\langle\left\{\hat{\Psi}_{C}\left(r_{1}, t\right)^{\dagger} \hat{\Psi}_{C}\left(s_{1}, t\right)\right\}\right\rangle$. By substituting we get $F[\underset{\rightarrow}{\rightarrow}]=\sum_{k} \alpha_{C k}^{+}(t) \phi_{C k}^{*}\left(r_{1}, t\right) \sum_{l} \alpha_{C l}(t) \phi_{C l}\left(s_{1}, t\right)=$ $\sum_{k l} \phi_{C k}^{*}\left(r_{1}, t\right) \alpha_{C k}^{+}(t) \alpha_{C l}(t) \phi_{C l}\left(s_{1}, t\right)$. The last equation is useful for working out the time derivatives of the various $F\left(\alpha, \alpha^{+}\right)=\alpha_{C k}^{+} \alpha_{C l}$, but it does not include the contribution to the time derivative of $F[\underline{\psi}]$ from the various $\phi_{C k}^{*}\left(r_{1}, t\right) \phi_{C l}\left(s_{1}, t\right)$. These contributions can of course be worked out and included later, but clearly the advantage of using field functions rather than separate phase variables is in terms of focusing directly on the quantum correlation functions that involve condensate and non-condensate field operators rather than just mode operators. As we will see there is no paradox associated with the missing term - there is a compensating term arising from the stochastic field average. 


\subsubsection{Stochastic Fields Average}

For the Ito stochastic field approach we now replace the fields $\psi_{A}^{\mu}$ by stochastic fields $\psi_{A}^{\mu s}$. We suppose $\psi_{A}^{\mu s}(x, t)$ satisfies an Ito stochastic field equation of the form

$$
\delta \psi_{A}^{\mu s}(x, t)=\mathcal{G}_{A}^{\mu}(x) \delta t+\sum_{a} \mathcal{N}_{A a}^{\mu}(x) \int_{t}^{t+\delta t} d t_{1} \Gamma_{a}\left(t_{1}\right)
$$

or equivalently

$$
\frac{\partial}{\partial t} \psi_{A}^{\mu s}(x, t)=\mathcal{G}_{A}^{\mu}(x)+\sum_{a} \mathcal{N}_{A a}^{\mu}(x) \Gamma_{a}\left(t_{+}\right)
$$

where expansions of $\psi_{A}^{\mu s}(x, t), \mathcal{G}_{A}^{\mu}(x)$ and $\mathcal{N}_{A a}^{\mu}(x)$ in terms of the mode functions $\phi_{A k}^{\mu}(x, t)$ and stochastic phases $\alpha_{\mu A k}^{s}(t)$ will be determined later.

Using an obvious generalisation of (189) we can write the change in the stochastic functional $F\left[\psi^{s}, \psi^{s+}\right]$ due to changes $\delta \psi_{A}^{\mu s}(x, t)$ in the stochastic fields as

$$
\begin{aligned}
& F\left[\psi^{s}(x, t)+\delta \psi^{s}(x, t), \psi^{s+}(x, t)+\delta \psi^{s+}(x, t)\right]-F\left[\psi^{s}(x, t), \psi^{s+}(x, t)\right] \\
= & \int d x \sum_{\mu A} \delta \psi_{A}^{\mu s}(x, t)\left(\frac{\delta F\left[\psi^{s}, \psi^{s+}\right]}{\delta \psi_{A}^{\mu s}(x, t)}\right)_{x} \\
& +\frac{1}{2} \iint d x d y \sum_{\mu A, \nu B} \delta \psi_{A}^{\mu s}(x, t) \delta \psi_{B}^{\nu s}(y, t)\left(\frac{\delta^{2} F\left[\psi^{s}, \psi^{s+}\right]}{\delta \psi_{A}^{\mu s}(x, t) \delta \psi_{B}^{\nu s}(y, t)}\right)_{x, y}
\end{aligned}
$$

correct to second order. Using the result that for any function $H\left(\psi_{A}^{\mu s}(x, t)\right)$ of the stochastic fields we have

$$
\begin{aligned}
& \overline{H\left(\psi_{A}^{\mu s}\left(x, t_{1}\right)\right) \Gamma_{a}\left(t_{2}\right) \Gamma_{b}\left(t_{3}\right) \Gamma_{c}\left(t_{4}\right) . . \Gamma_{k}\left(t_{l}\right)} \\
& =\overline{H\left(\psi_{A}^{\mu s}\left(x, t_{1}\right)\right)} \overline{\Gamma_{a}\left(t_{2}\right) \Gamma_{b}\left(t_{3}\right) \Gamma_{c}\left(t_{4}\right) . . \Gamma_{k}\left(t_{l}\right)} \quad t_{1}<t_{2}, t_{3}, . ., t_{l}
\end{aligned}
$$

we can then evaluate $\overline{F\left[\psi^{s}(x, t)+\delta \psi^{s}(x, t), \psi^{s+}(x, t)+\delta \psi^{s+}(x, t)\right]-F\left[\psi^{s}(x, t), \psi^{s+}(x, t)\right]}$.

For the first order derivative terms we have after substituting from (139), expanding and carrying out the stochastic averaging

$$
=\overline{\int d x\left\{\sum_{\mu A} \delta \psi_{A}^{\mu s}(x, t)\left(\frac{\delta F\left[\psi^{s}, \psi^{s+}\right]}{\delta \psi_{A}^{\mu s}(x, t)}\right)_{x}\right\}}
$$

where the stochastic average rules for sums and products have been used, the non-correlation between the averages of functions of $\psi_{\mu}^{s}(x, t)$ at time $t$ and the $\Gamma$ at later times between $t$ to $t+\delta t$ is applied and the term involving $\overline{\Gamma_{a}\left(t_{1}\right)}$ is 
equal to zero from (78). Note that this term is proportional to $\delta t$. Details are set out in Appendix 10.

For the second order derivative terms we have after substituting from (139), expanding and carrying out the stochastic averaging

$$
\begin{aligned}
& \overline{\left\{\frac{1}{2} \iint d x d y \sum_{\mu A, \nu B} \delta \psi_{A}^{\mu s}(x, t) \delta \psi_{B}^{\nu s}(y, t)\left(\frac{\delta^{2} F\left[\psi^{s}, \psi^{s+}\right]}{\delta \psi_{A}^{\mu s}(x, t) \delta \psi_{B}^{\nu s}(y, t)}\right)_{x, y}\right\}} \\
& =\overline{\frac{1}{2} \iint d x d y \sum_{\mu A, \nu B}\left(\frac{\delta^{2} F\left[\psi^{s}, \psi^{s+}\right]}{\delta \psi_{A}^{\mu s}(x, t) \delta \psi_{B}^{\nu S}(y, t)}\right)_{x, y}\left[\sum_{a} \mathcal{N}_{A a}^{\mu}(x) \mathcal{N}_{B a}^{\nu}(y)\right]} \delta t \\
& =\overline{\frac{1}{2} \iint d x d y \sum_{\mu A, \nu B}\left(\frac{\delta^{2} F\left[\psi^{s}, \psi^{s+}\right]}{\delta \psi_{A}^{\mu s}(x, t) \delta \psi_{B}^{\nu s}(y, t)}\right)_{x, y}\left[\left[\mathcal{N}(x) \mathcal{N}^{T}(y)\right]_{A, B}^{\mu, \nu}\right]} \delta t
\end{aligned}
$$

where the stochastic average rules for sums and products have been used, the non-correlation between the averages of functions of $\psi_{\mu}^{s}(x, t)$ at time $t$ and the $\Gamma$ at later times between $t$ to $t+\delta t$ is applied. The terms involving a single $\Gamma$ have a zero stochastic average, whilst from Eq.(78) the terms with two $\Gamma$ give a stochastic average proportional to $\delta t$. In evaluating the latter term Eq. (81) is used. Details are set out in Appendix 10.

The remaining terms give stochastic averages correct to order $\delta t^{2}$ or higher so that we have correct to first order in $\delta t$

$$
\begin{aligned}
& =\overline{F\left[\psi^{s}(x, t)+\delta \psi^{s}(x, t), \psi^{s+}(x, t)+\delta \psi^{s+}(x, t)\right]}-\overline{F\left[\psi^{s}(x, t), \psi^{s+}(x, t)\right]} \\
& =\left\{\overline{\left.\int d x \sum_{\mu A}\left(\frac{\delta F\left[\psi^{s}, \psi^{s+}\right]}{\delta \psi_{A}^{\mu s}(x, t)}\right)_{x} \mathcal{G}_{A}^{\mu}(x)\right\} \delta t}\right. \\
& +\left\{\overline{\frac{1}{2} \iint d x d y \sum_{\mu A, \nu B}\left(\frac{\delta^{2} F\left[\psi^{s}, \psi^{s+}\right]}{\delta \psi_{A}^{\mu s}(x, t) \delta \psi_{v}(y, t)}\right)_{x, y}\left[\left[\mathcal{N}(x) \mathcal{N}^{T}(y)\right]_{A, B}^{\mu, \nu}\right]}\right\} \delta t
\end{aligned}
$$

or

$$
\begin{aligned}
& \frac{\partial}{\partial t} \overline{F\left[\psi^{s}(x, t), \psi^{s+}(x, t)\right]} \\
& =\overline{\int d x \sum_{\mu A}\left(\frac{\delta F\left[\psi^{s}, \psi^{s+}\right]}{\delta \psi_{A}^{\mu s}(x, t)}\right)_{x} \mathcal{G}_{A}^{\mu}(x)} \\
& +\overline{\frac{1}{2} \iint d x d y \sum_{\mu A, \nu B}\left(\frac{\delta^{2} F\left[\psi^{s}, \psi^{s+}\right]}{\delta \psi_{A}^{\mu s}(x, t) \delta \psi_{v}(y, t)}\right)_{x, y}\left[\left[\mathcal{N}(x) \mathcal{N}^{T}(y)\right]_{A, B}^{\mu, \nu}\right]}
\end{aligned}
$$

For the phase space functional average (137) and the stochastic average (146) 
to agree we require two conditions to be satisfied, as follows.

$$
\begin{aligned}
& \mathcal{G}_{A}^{\mu}(x)=A_{A}^{\mu}(x) \\
+ & \int d y \sum_{B}\left(\sum_{k l} \phi_{A k}^{\mu}(x, t) C_{A k B l}^{\mu} \phi_{B l}^{-\mu}(y, t)\right) \psi_{B}^{\mu}(y, t) \\
& +\int d y \sum_{B}\left(\sum_{k l} \phi_{B l}^{\mu}(x, t) C_{A k B l}^{-\mu} \phi_{A k}^{-\mu}(y, t)\right) \psi_{A}^{\mu}(y, t) \\
= & D_{A B}^{\mu \nu}(x, y) \\
+ & \frac{1}{2}\left\{\delta_{A C} \delta_{B N C} \delta_{\mu-\nu}\left(\sum_{k} \sum_{l} \phi_{A k}^{\mu}(x, t) C_{A k B l}^{\mu}(t) \phi_{B l}^{\nu}(y, t)\right)\right\} \\
+ & \frac{1}{2}\left\{\delta_{B C} \delta_{A N C} \delta_{\nu-\mu}\left(\sum_{k} \sum_{l} \phi_{B l}^{\nu}(y, t) C_{B l A k}^{\nu}(t) \phi_{A k}^{\mu}(x, t)\right)\right\}
\end{aligned}
$$

These conditions determine the quantities $\mathcal{G}_{A}^{\mu}(x)$ and $\mathcal{N}_{A a}^{\mu}(x)$ that occur in the Ito stochastic field equations (139) in terms of the quantities that occur in the functional Fokker-Planck equation (129). Note that the $B=A$ terms in the result for $\mathcal{G}_{A}^{\mu}(x)$ actually cancel out because $C_{A k A l}^{\mu}+C_{A l A k}^{-\mu}=0$. These relationships are to be interpreted as replacing the stochastic quantities $\mathcal{G}_{A}^{\mu}(x)$, $\mathcal{N}_{A a}^{\mu}(x)$ in the Ito stochastic field equation (140) with the quantities $A_{A}^{\mu}(x)$, $N_{A a}^{\mu}(x)$ and $\psi_{A}^{\mu}(x, t)$ originating from the functional Fokker-Planck equation (129), which are then regarded as depending on the stochastic fields $\psi_{\mu}^{s}(x, t)$.

We next show that these conditions can always be satisfied. In Appendix 7 we have shown that the Fokker-Planck equation for the distribution function and the functional Fokker-Planck equation for the distribution functional are equivalent, and we can make use of the results obtained there.

First, we consider the condition for $\mathcal{G}_{A}^{\mu}(x)$. From (203) the drift term $A_{A}^{\mu}(x)$ in the functional Fokker-Planck equation is related to the drift vector $A_{A k}^{\mu}$ in the Fokker-Planck equation via

$$
\begin{aligned}
A_{A}^{\mu}(x) & =\sum_{k} \phi_{A k}^{\mu}(x, t) A_{A k}^{\mu} \\
A_{A k}^{\mu} & =\int d x \phi_{A k}^{-\mu}(x, t) A_{A}^{\mu}(x)
\end{aligned}
$$


We can also use Eqs. (115), (116) and (60) to show that

$$
\begin{aligned}
& \int d y \sum_{B}\left(\sum_{k l} \phi_{A k}^{\mu}(x, t) C_{A k B l}^{\mu} \phi_{B l}^{-\mu}(y, t)\right) \psi_{B}^{\mu}(y, t) \\
& +\int d y \sum_{B}\left(\sum_{k l} \phi_{B l}^{\mu}(x, t) C_{A k B l}^{-\mu} \phi_{A k}^{-\mu}(y, t)\right) \psi_{A}^{\mu}(y, t) \\
= & \left(\sum_{k} \phi_{A k}^{\mu}(x, t) \sum_{B l} C_{A k B l}^{\mu} \alpha_{\mu B l}\right)+\left(\sum_{k} \frac{\partial}{\partial t} \phi_{A k}^{\mu}(x, t) \alpha_{\mu A k}\right)
\end{aligned}
$$

Clearly the first condition is satisfied via the choice

$\mathcal{G}_{A}^{\mu}(x)=\sum_{k} \phi_{A k}^{\mu}(x, t) A_{A k}^{\mu}+\sum_{k} \phi_{A k}^{\mu}(x, t) \sum_{B l} C_{A k B l}^{\mu} \alpha_{\mu B l}^{s}+\sum_{k} \frac{\partial}{\partial t} \phi_{A k}^{\mu}(x, t) \alpha_{\mu A k}^{s}$

written in terms of mode functions and quantities derived from the FokkerPlanck equation (63).

Second, for the condition for $\mathcal{N}_{A a}^{\mu}(x)$ we need to be able to find $\mathcal{N}_{A a}^{\mu}(x)$ such that

$$
\sum_{a} \mathcal{N}_{A a}^{\mu}(x) \mathcal{N}_{B a}^{\nu}(y)=E_{A B}^{\mu \nu}(x, y)
$$

Using the relationship in Eq.(203) between the diffusion matrix $E_{A B}^{\mu \nu}(x, y)$ in the functional Fokker-Planck equation (129) and the diffusion matrix $E_{A k B l}^{\mu \nu}$ in the Fokker-Planck equation (63) we have

$$
\begin{aligned}
E_{A B}^{\mu \nu}(x, y) & =\sum_{k l} \phi_{A k}^{\mu}(x, t) E_{A k B l}^{\mu \nu} \phi_{B l}^{\nu}(y, t) \\
E_{A k B l}^{\mu \nu} & =\iint d x d y \phi_{A k}^{-\mu}(x, t) E_{A B}^{\mu \nu}(x, y) \phi_{B l}^{-\nu}(y, t)
\end{aligned}
$$

The matrix $E$ is symmetric $\left(E_{A k B l}^{\mu \nu}=E_{B l A k}^{\nu \mu}\right)$, so from the Takagi factorisation [45, [46 it can be written as $K K^{T}$, where the matrix $K$ is as in Section 2 . Eq. (86). Thus the diffusion matrix $E_{A B}^{\mu \nu}(x, y)$ may be factorised as

$$
E_{A B}^{\mu \nu}(x, y)=\sum_{a} N_{A a}^{\mu}(x) N_{B a}^{\nu}(y)
$$

where

$$
\begin{aligned}
N_{A a}^{\mu}(x) & =\sum_{k} K_{A k a}^{\mu} \phi_{A k}^{\mu}(x, t) \\
E_{A k B l}^{\mu \nu} & =\sum_{a} K_{A k a}^{\mu} K_{B l a}^{\nu}
\end{aligned}
$$


Hence the second condition is satisfied with the choice

$$
\begin{aligned}
\mathcal{N}_{A a}^{\mu}(x) & =N_{A a}^{\mu}(x) \\
& =\sum_{k} K_{A k a}^{\mu} \phi_{A k}^{\mu}(x, t)
\end{aligned}
$$

again written in terms of mode functions and quantities derived from the FokkerPlanck equation (63).

Finally, we can show that the Ito stochastic field equations (140) for the stochastic fields $\psi_{A}^{\mu s}(x, t)$ in the field theory treatment are entirely equivalent to the Ito stochastic equations (87) for the stochastic phase variables $\alpha_{\mu A k}^{s}$ in the separate mode treatment. The proof is given in Appendix 7 .

This proof shows that the stochastic fields are given by

$$
\psi_{A}^{\mu s}(x, t)=\sum_{k} \alpha_{\mu A k}^{s}(t) \phi_{A k}^{\mu}(x, t)
$$

where the stochastic feature results only from the phase variables being replaced by stochastic variables. Thus the stochastic field equations for $\psi_{A}^{\mu s}(x, t)$ are equivalent to the stochastic phase variable equations for the $\alpha_{\mu A k}^{s}(t)$.

\subsection{Expressions for Ito Stochastic Field Equations}

The original Ito stochastic field equation (140) can also be written in terms of the drift, diffusion terms that are derived directly from the functional FokkerPlanck equation (129) itself. Substituting from (156) and (147) we have for the Ito stochastic field equation

$$
\begin{aligned}
& \frac{\partial}{\partial t} \psi_{A}^{\mu s}(x, t)=A_{A}^{\mu}(x) \\
& +\int d y \sum_{B \neq A}\left(\sum_{k l} \phi_{A k}^{\mu}(x, t) C_{A k B l}^{\mu} \phi_{B l}^{-\mu}(y, t)\right) \psi_{B}^{\mu s}(y, t) \\
& +\int d y \sum_{B \neq A}\left(\sum_{k l} \phi_{A k}^{-\mu}(y, t) C_{A k B l}^{-\mu} \phi_{B l}^{\mu}(x, t)\right) \psi_{A}^{\mu s}(y, t) \\
& +\sum_{a} N_{A a}^{\mu}(x) \Gamma_{a}\left(t_{+}\right)
\end{aligned}
$$


where $A_{A}^{\mu}(x)$ is the drift term and $N_{A a}^{\mu}(x)$ is related to the diffusion term $E_{A B}^{\mu \nu}(x, y)$ that occur in the functional Fokker-Planck equation (129) via

$$
\begin{aligned}
\sum_{a} N_{A a}^{\mu}(x) N_{B a}^{\mu}(y)= & E_{A B}^{\mu \nu}(x, y) \\
= & D_{A B}^{\mu \nu}(x, y) \\
& +\frac{1}{2}\left\{\delta_{A C} \delta_{B N C} \delta_{\mu-\nu}\left(\sum_{k} \sum_{l} \phi_{A k}^{\mu}(x, t) C_{A k B l}^{\mu}(t) \phi_{B l}^{\nu}(y, t)\right)\right\} \\
& +\frac{1}{2}\left\{\delta_{B C} \delta_{A N C} \delta_{\nu-\mu}\left(\sum_{k} \sum_{l} \phi_{B l}^{\nu}(y, t) C_{B l A k}^{\nu}(t) \phi_{A k}^{\mu}(x, t)\right)\right\}
\end{aligned}
$$

The $B=A$ terms have been cancelled out using $C_{A k A l}^{\mu}+C_{A k A l}^{-\mu}=0$. The result for $\frac{\partial}{\partial t} \psi_{A}^{\mu s}(x, t)$ may be compared to the Ito stochastic equation (87) for the phase variables. The term that is added to $A_{A}^{\mu}(x)$ involves a non-local contribution with a kernel that depends on the mode functions and their time dependence via the coupling coefficients. It has the effect of coupling the condensate stochastic fields $\psi_{C}^{\mu s}$ to the non-condensate stochastic fields $\psi_{N C}^{\mu s}(\mu=-,+)$. This term is not present when time independent modes are used. Note that it is of the same form as in (117) for the time derivative of the (non-stochastic) field functions $\psi_{A}^{\mu}(x, t)$. This form for the Ito stochastic field equation is the most useful, since it involves the drift $A_{A}^{\mu}(x)$ and diffusion terms $D_{A B}^{\mu \nu}(x, y)$ in the standard functional Fokker-Planck equation obtained by applying the correspondence rules for the $\frac{\partial}{\partial t} \hat{\rho}$ contribution in the characteristic functional, plus the time dependent modes $\phi_{A k}^{\mu}(x, t)$ and the coupling coefficients $C_{A k B l}^{\mu}(t)$.

\subsection{Classical and Noise Field Terms}

We can write the Ito stochastic field equation in terms of a classical term and a noise term

$$
\begin{aligned}
\frac{\partial}{\partial t} \psi_{A}^{\mu s}(x, t)= & \left(\frac{\partial}{\partial t} \psi_{A}^{\mu s}(x, t)\right)_{\text {class }}+\left(\frac{\partial}{\partial t} \psi_{A}^{\mu s}(x, t)\right)_{\text {noise }} \\
\left(\frac{\partial}{\partial t} \psi_{A}^{\mu s}(x, t)\right)_{\text {class }}= & A_{A}^{\mu}(x) \\
& +\int d y \sum_{B \neq A}\left(\sum_{k l} \phi_{A k}^{\mu}(x, t) C_{A k B l}^{\mu} \phi_{B l}^{-\mu}(y, t)\right) \psi_{B}^{\mu s}(y, t) \\
& +\int d y \sum_{B \neq A}\left(\sum_{k l} \phi_{A k}^{-\mu}(y, t) C_{A k B l}^{-\mu} \phi_{B l}^{\mu}(x, t)\right) \psi_{A}^{\mu s}(y, t) \\
\left(\frac{\partial}{\partial t} \psi_{A}^{\mu s}(x, t)\right)_{\text {noise }}= & \sum_{a} N_{A a}^{\mu}(x) \Gamma_{a}\left(t_{+}\right)
\end{aligned}
$$

If only the classical terms were included, then the solution for $\psi_{A}^{\mu s}(x, t)$ would determine classical field functions; hence their name. Note that the number of 
noise terms will be equal to $2\left(n_{C}+n_{N C}\right)$, or twice the total number of condensate and non-condensate modes.

\subsection{Properties of Noise Fields}

The stochastic averages of the noise field terms can now be evaluated. For a single noise term and the product of two noise terms we have using Eqs. (78) and (142)

$$
\begin{aligned}
\overline{\left(\frac{\partial}{\partial t} \psi_{\mu A}^{s}(x, t)\right)_{n}} & =0 \\
\overline{\left(\frac{\partial}{\partial t} \psi_{\mu A}^{s}\left(x_{1}, t_{1}\right)\right)_{n}\left(\frac{\partial}{\partial t} \psi_{\nu B}^{s}\left(x_{2}, t_{2}\right)\right)_{n}} & =\delta\left(t_{1}-t_{2}\right) \overline{E_{A B}^{\mu \nu}\left(x_{1}, x_{2} ; t_{1,2}\right)}(161)
\end{aligned}
$$

showing that the stochastic average of a single noise field term is zero whilst that for the product of two noise field terms is delta correlated in time and equal to the appropriate diffusion matrix element. Note that it is not necessarily delta correlate in space.

In fact the diffusion matrix elements determine all the stochastic averages of products of noise field terms. With an odd number of terms the stochastic average is zero. For an even number of terms the stochastic average involves sums of stochastic averages of products of diffusion matrix elements, which reflects the Gaussian-Markoff properties of the $\Gamma_{a}$. Thus for three and four noise field terms

$$
\begin{aligned}
& \overline{\left(\frac{\partial}{\partial t} \psi_{\mu A}^{s}\left(x_{1}, t_{1}\right)\right)_{n}\left(\frac{\partial}{\partial t} \psi_{\nu B}^{s}\left(x_{2}, t_{2}\right)\right)_{n}\left(\frac{\partial}{\partial t} \psi_{\xi C}^{s}\left(x_{3}, t_{3}\right)\right)_{n}}=0 \\
& \overline{\left(\frac{\partial}{\partial t} \psi_{\mu A}^{s}\left(x_{1}, t_{1}\right)\right)_{n}\left(\frac{\partial}{\partial t} \psi_{\nu B}^{s}\left(x_{2}, t_{2}\right)\right)_{n}\left(\frac{\partial}{\partial t} \psi_{\xi C}^{s}\left(x_{3}, t_{3}\right)\right)_{n}\left(\frac{\partial}{\partial t} \psi_{\lambda D}^{s}\left(x_{4}, t_{4}\right)\right)_{n}} \\
= & \delta\left(t_{1}-t_{2}\right) \delta\left(t_{3}-t_{4}\right) \frac{E_{A B}^{\mu \nu}\left(x_{1}, x_{2} ; t_{1,2}\right) E_{C D}^{\xi \lambda}\left(x_{3}, x_{4} ; t_{3,4}\right)}{E_{A C}^{\mu \xi}\left(x_{1}, x_{3} ; t_{1,3}\right) E_{B D}^{\nu \lambda}\left(x_{2}, x_{4} ; t_{2,4}\right)} \\
& +\delta\left(t_{1}-t_{3}\right) \delta\left(t_{2}-t_{4}\right) \\
& +\delta\left(t_{1}-t_{4}\right) \delta\left(t_{2}-t_{3}\right) \frac{E_{A D}^{\mu \lambda}\left(x_{1}, x_{4} ; t_{1,4}\right) E_{B C}^{\nu \xi}\left(x_{2}, x_{3} ; t_{2,3}\right)}{}
\end{aligned}
$$

Note that the noise field terms are not themselves Gaussian-Markoff processes. 


\section{Conclusions}

This paper has set out a phase space theory approach to treating the dynamical behaviour of Bose-Einstein condensates which could be applied to a variety of experimental situations, including interferometry with BEC involving timedependent double well potentials. The key feature is the use of time-dependent mode functions, chosen so that a few (one, two,..) highly occupied modes provide a good approximation to the physics of interacting bosons in the time dependent potential at temperatures well below the BEC transition tememperature. The motivation is that when the effect the lightly occupied noncondensate modes are taken into account in treatments of decoherence effects allowing for Bogoliubov excitations, collisions with thermal bosons etc., the previously determined condensate modes are still important in treating these extensions. To allow for time dependent mode functions, the major innovation here is the introduction of time dependent phase variables, the time dependence being chosen to match that of the time dependent mode annihilation, creation operators. This means that in this approach we are representing time dependent mode annihilation, creation operators by time dependent phase variables, whilst time independent total field annihilation, creation operators are represented by time independent field functions. In the more usual approach using time independent phase variables the opposite situation applies, which is rather unsatisfactory. The theory presented here treats the two situations, one (mode theory) being where mode annihilation, creation operators and their related phase variables and distribution functions are dealt with specifically, the other (field theory) being where field creation, annihilation operators and their related field functions and distribution functionals involve a description where individual modes are not distinguished. Though each situation is treated separately, they are shown to be equivalent. Within each of these two situations the modes are divided up between condensate (highly occupied) modes and non-condensate (sparsely occupied) modes. This is referred to as the hybrid approach. For the hybrid case a Wigner type distribution function or functional is used for the condensate bosons and a positive $\mathrm{P}$ type for the non-condensate bosons. Fokker-Planck or functional Fokker-Planck equations are derived, along with the Ito stochastic equations for stochastic phases or stochastic fields, and the relationship between the Ito equation quantities and those in the FokkerPlanck equation is obtained. The stochastic equations contain the sum of a classical term related to the drift vector and a noise term, and the stochastic properties of the noise terms are related to the diffusion matrix. Also, the expressions for the stochastic fields involve the same expansion in terms of time dependent mode functions and stochastic phase variables, as had applied to the field function expansions involving non-stochastic phase variables.

The key results are set out below. For the hybrid approach the condensate and non-condensate field operators are time dependent, as are the corresponding field functions. Also, both the Fokker-Planck and functional Fokker-Planck equations differ from those derived using the usual correspondence rules, the

drift vectors are unchanged but the diffusion matrices contain additional terms 
relating to the time dependence of the mode functions and involving the coupling coefficients, which are defined via overlap integrals between mode functions and their time derivatives. In addition, there are extra terms in the Ito stochastic equations both for the stochastic phases and stochastic fields, again related to the time dependence of the mode functions and involving the coupling coefficients. For the hybrid case the final form for the Ito stochastic field equation in Eqs. (164), (165) is the most useful, since it involves the drift $A_{A}^{\mu}(x)$ and diffusion terms $D_{A B}^{\mu \nu}(x, y)$ in the standard functional Fokker-Planck equation obtained by applying the correspondence rules for the $\frac{\partial}{\partial t} \hat{\rho}$ contribution in the characteristic functional, plus the time dependent modes $\phi_{A k}^{\mu}(x, t)$ and the coupling coefficients $C_{A k B l}^{\mu}(t)$.

From the point of view of best describing the physics of BECs even near zero temperature the treatment preferred here is the hybrid one involving condensate and non-condensate fields rather than considering a large number of separate modes, but based on time dependent mode functions. For this reason, the field theory treatment has been given an important emphasis in this paper. That treatment of the hybrid approach capitalises on the simple but accurate picture based on highly occupied condensate modes and lightly occupied non-condensate modes, but avoids a mode-by-mode approach in determining the quantum correlation functions - which after all only involve field operators. There are of course some costs, for numerical work the set of time dependent mode functions and the coupling constants would need to be worked out first, but hopefully only a few condensate modes would be needed and the non-condensate modes might be introduced via physically based variational methods involving Fock states where only one boson is in a non-condensate mode, or by just applying Schmidt orthogonalisation procedures.

In Ref. 14 the hybrid approach was applied based on including time dependent condensate modes and the functional Fokker-Planck equations derived using the correspondence rules as set out here in Eqs. (125) and (126). In terms of this paper (see Eq. (166)) this gives the drift vector $A_{A}^{\mu}(x)$ and the diffusion matrix contribution $D_{A B}^{\mu \nu}(x, y)$. However, no terms were included in either the functional Fokker-Planck equation or the Ito stochastic field equations involving the coupling coefficients $C_{A k B l}^{\mu}(t)$. The theory in Ref. [14] was based on using time independent phase variables and characteristic function variables, but the time dependences of both the condensate and non-condensate field operators and field functions consequential on using time dependent condensate modes were ignored. Eqs. (192), (193) and (207) in Ref. [14] do not include contributions involving the coupling coefficients. The results for the Ito stochastic field equations in Ref. 14] are correct if time independent (or slowly varying) condensate and non-condensate modes were used, but then the condensate would require many more than one or two modes for an adequate description. Note however that the non-local term involving $F(\mathbf{r}, \mathbf{s})$ in the diffusion matrices in Ref. [14 would still be present, as this arose from the $\frac{\partial}{\partial t} \hat{\rho}$ contribution in the characteristic functional and does not involve mode time dependence. However, results from the present paper are really needed to supplement those in Ref. [14] if the physically based idea of just having one or two genuinely time-dependent 
condensate modes is to be implemented.

The results in this paper can be easily converted back to the corresponding results based on using a convenient set of time independent mode functions. In this case all the coupling constants become zero and the phase variables, mode and field operators become time independent again. The functional FokkerPlanck and Fokker-Planck equations would revert to those obtainable by standard methods using the correspondence rules, the Ito stochastic equations for fields and phase variables are also the same as in standard methods, as also are the relationships between these equations and the Fokker-Planck equations. However, such mode functions are not closely aligned to those that provide a good approximation to the near zero temperature physics, so any advantages in terms of simplicity in applying the theory and convenience for numerical work is likely to be negated.

As well as the hybrid approach treated in this paper, situations where time dependent mode functions are involved may also be treated using the so-called combined approach, where the modes are not divided up into condensate or non-condensate types and only the total field operators and field functions are considered. For completeness, the results for the combined approach are also set out below. The same method involving time dependent phase space variables can be used. Again, two situations may be considered, one (mode theory) being where mode annihilation, creation operators and their related phase variables and distribution functions are dealt with specifically, the other (field theory) being where total field creation, annihilation operators and their related field functions and distribution functionals involve a description where individual modes are not distinguished. The distribution function or functional may be either of the Wigner or positive $\mathrm{P}$ type. For the combined approach both the Fokker-Planck and functional Fokker-Planck equations are exactly the same as those derived using the usual correspondence rules, even though time dependent modes are present. The field functions are time independent. However, there are extra terms in the Ito stochastic equations for the stochastic phases though not for the stochastic fields. These terms relate to the time dependence of the mode functions and involve the coupling coefficients. For the combined case, the final form for the Ito stochastic field equation in Eqs. (171) is the most useful, since it involves the drift $A^{\mu}(x)$ and diffusion terms $D^{\mu \nu}(x, y)$ in the standard functional Fokker-Planck equation obtained by applying the correspondence rules for the $\frac{\partial}{\partial t} \hat{\rho}$ contribution in the characteristic functional, plus the time dependent modes $\phi_{k}^{\mu}(x, t)$ and the coupling coefficients $C_{k l}^{\mu}(t)$. 


\section{$5 \quad$ Summary of Key Results}

\subsection{Hybrid Approach - Condensate and Non-Condensate Modes and Fields}

Here we summarise the key results obtained in this paper. Results for functional Fokker-Planck, Ito stochastic field equations and the Fokker-Planck, Ito stochastic phase variable equations for the hybrid approach where the time dependent modes are divided into $n_{C}$ condensate modes and $n_{N C}$ non-condensate modes, plus conversions and key results are as follows.

For the field theory results:

$$
\begin{aligned}
& \frac{\partial}{\partial t} P\left[\underline{\psi}_{\rightarrow}, \underline{\psi}^{*}\right]=\left\{-\sum_{\mu A} \int d x \frac{\delta}{\delta \psi_{A}^{\mu}(x, t)} A_{A}^{\mu}(x)\right\} P\left[\underline{\psi}, \underline{\psi}^{*}\right] \\
& +\left\{\frac{1}{2} \sum_{\mu A} \sum_{\nu B} \iint d x d y \frac{\delta}{\delta \psi_{A}^{\mu}(x, t)} \frac{\delta}{\delta \psi_{B}^{\nu}(y, t)} E_{A B}^{\mu \nu}(x, y)\right\} P\left[\stackrel{\sim}{\rightarrow}, \underline{\psi}^{*}\right] \\
& \psi_{A}^{\mu}(x, t)=\sum_{k} \alpha_{\mu A k}(t) \phi_{A k}^{\mu}(x, t) \quad \alpha_{\mu A k}=\int d y \phi_{A k}^{-\mu}(y, t) \psi_{A}^{\mu}(y, t) \\
& \frac{\partial}{\partial t} \psi_{A}^{\mu s}(x, t)=A_{A}^{\mu}(x)+\int d y \sum_{B \neq A}\left(\sum_{k l} \phi_{A k}^{\mu}(x, t) C_{A k B l}^{\mu} \phi_{B l}^{-\mu}(y, t)\right) \psi_{B}^{\mu s}(y, t) \\
& +\int d y \sum_{B \neq A}\left(\sum_{k l} \phi_{A k}^{-\mu}(y, t) C_{A k B l}^{-\mu} \phi_{B l}^{\mu}(x, t)\right) \psi_{A}^{\mu s}(y, t) \\
& +\sum_{a} N_{A a}^{\mu}(x) \Gamma_{a}\left(t_{+}\right) \\
& E_{A B}^{\mu \nu}(x, y)=D_{A B}^{\mu \nu}(x, y) \\
& +\frac{1}{2}\left\{\delta_{A C} \delta_{B N C} \delta_{\mu-\nu}\left(\sum_{k} \sum_{l} \phi_{A k}^{\mu}(x, t) C_{A k B l}^{\mu}(t) \phi_{B l}^{\nu}(y, t)\right)\right\} \\
& +\frac{1}{2}\left\{\delta_{B C} \delta_{A N C} \delta_{\nu-\mu}\left(\sum_{k} \sum_{l} \phi_{B l}^{\nu}(y, t) C_{B l A k}^{\nu}(t) \phi_{A k}^{\mu}(x, t)\right)\right\} \\
& =\sum_{a} N_{A a}^{\mu}(x) N_{B a}^{\mu}(y) \\
& \psi_{A}^{\mu s}(x, t)=\sum_{k} \alpha_{\mu A k}^{s}(t) \phi_{A k}^{\mu}(x, t) \\
& \alpha_{\mu A k}^{s}=\int d y \phi_{A k}^{-\mu}(y, t) \psi_{A}^{\mu s}(y, t)(1
\end{aligned}
$$


For the mode theory results:

$$
\begin{aligned}
\frac{\partial}{\partial t} P\left(\alpha, \alpha^{+}, \alpha^{*}, \alpha^{+*}\right) & =\left\{-\sum_{\mu A k} \frac{\partial}{\partial \alpha_{\mu A k}} A_{A k}^{\mu}+\frac{1}{2} \sum_{\mu A k} \sum_{\nu B l} \frac{\partial}{\partial \alpha_{\mu A k}} \frac{\partial}{\partial \alpha_{\nu B l}} E_{A k B l}^{\mu \nu}\right\} P\left(\alpha, \alpha^{+}, \alpha^{*}, \alpha^{+*}\right) \\
\frac{\partial}{\partial t} \alpha_{\mu A k}^{s} & =A_{A k}^{\mu}+\sum_{B l} C_{A k B l}^{\mu} \alpha_{\mu B l}^{s}+\sum_{a} K_{A k a}^{\mu} \Gamma_{a}\left(t_{+}\right) \\
E_{A k B l}^{\mu \nu} & =D_{A k B l}^{\mu \nu}+\frac{1}{2}\left(\delta_{A C} \delta_{B N C} \delta_{\mu-\nu} C_{A k B l}^{\mu}+\delta_{B C} \delta_{A N C} \delta_{\nu-\mu} C_{B l A k}^{\nu}\right) \\
& =\sum_{a} K_{A k a}^{\mu} K_{B l a}^{\nu}
\end{aligned}
$$

Conversions between the quantities are as follows

$$
\begin{aligned}
A_{A k}^{\mu} & =\int d x \phi_{A k}^{-\mu}(x, t) A_{A}^{\mu}(x) \quad A_{A}^{\mu}(x)=\sum_{k} \phi_{A k}^{\mu}(x, t) A_{A k}^{\mu} \\
E_{A k B l}^{\mu \nu} & =\iint d x d y \phi_{A k}^{-\mu}(x, t) E_{A B}^{\mu \nu}(x, y) \phi_{B l}^{-\nu}(y, t) \quad E_{A B}^{\mu \nu}(x, y)=\sum_{k l} \phi_{A k}^{\mu}(x, t) E_{A k B l}^{\mu \nu} \phi_{B l}^{\nu}(y, t) \\
N_{A a}^{\mu}(x) & =\sum_{k} K_{A k a}^{\mu} \phi_{A k}^{\mu}(x, t)
\end{aligned}
$$

Key results for the time dependences are

$$
\begin{aligned}
\frac{\partial}{\partial t} \phi_{A k}^{\mu}(x, t) & =\sum_{B l} C_{A k B l}^{-\mu} \phi_{B l}^{\mu}(x, t) \\
\frac{\partial}{\partial t} \alpha_{\mu A k} & =\sum_{B l} C_{A k B l}^{\mu} \alpha_{\mu B l}
\end{aligned}
$$

with coupling coefficients

$$
\begin{aligned}
C_{A k B l}^{-}(t) & =\int d x \frac{\partial \phi_{A k}^{*}(x, t)}{\partial t} \phi_{B l}(x, t)=C_{A k B l}(t) \\
C_{A k B l}^{+}(t) & =\int d x \frac{\partial \phi_{A k}(x, t)}{\partial t} \phi_{B l}^{*}(x, t)=C_{A k B l}^{*}(t)
\end{aligned}
$$

\subsection{Combined Approach - Modes and Fields}

A much simpler treatment applies if all $n$ condensate and non-condensate modes are considered together in the so-called combined approach, even though the mode functions are time dependent. Here we will just summarise the results for completeness, but without proof since the proofs are easily obtained from the previous sections. Many of the extra terms for the hybrid case just become zero. The phase variables are again considered to be time dependent, as in Eq. (37) as are the characteristic function variables, as in Eq. (41). The phase space treatment is based on the positive $\mathrm{P}$ distribution for all modes, but similar results would apply if the double space Wigner representation was used. It then 
turns out that the Fokker-Planck equations involving the phase variables $\alpha_{\mu k}$ $(\mu=-,+, k=1, . ., n)$ have the same drift $A_{k}^{\mu}$ and diffusion $D_{k l}^{\mu \nu}$ terms as in the standard Fokker-Planck equations resulting from just the $\frac{\partial}{\partial t} \hat{\rho}$ contribution in the characteristic function. However, the Ito stochastic equations have an additional contribution $\sum_{l} C_{k l}^{\mu} \alpha_{\mu l}^{s}$ to the classical field term, though the noise term just involves the matrix $B$ related to the diffusion matrix via $D=B B^{T}$. The functional Fokker-Planck for the time independent total fields $\psi_{\mu}(x)$ turns out to be exactly the same as in the standard functional Fokker-Planck equations resulting from just the $\frac{\partial}{\partial t} \hat{\rho}$ contribution in the characteristic functional, involving drift $A^{\mu}(x)$ and diffusion $D^{\mu \nu}(x, y)$ terms. Also, the Ito stochastic field equations are of the same form as if the mode functions were time independent. The classical field term is given by the drift term and the noise field term is related in the standard way to the diffusion term in the functional FokkerPlanck equation. Furthermore, the stochastic field $\psi_{\mu}^{s}(x, t)$ can be expanded in terms of the time dependent mode functions with the stochastic phase variables as coefficients, with the same form as for the non-stochastic fields $\psi_{\mu}(x)$.

Results for functional Fokker-Planck, Ito stochastic field equations and the Fokker-Planck, Ito stochastic phase variable equations for the combined approach where all time dependent modes are considered together, plus conversions and key results are as follows.

For the field theory results:

$$
\begin{aligned}
\frac{\partial}{\partial t} P\left[\psi, \psi^{+}, \psi^{*}, \psi^{+*}\right]= & \left\{-\sum_{\mu} \int d x \frac{\delta}{\delta \psi_{\mu}(x)} A^{\mu}(x)\right\} P\left[\psi, \psi^{+}, \psi^{*}, \psi^{+*}\right] \\
& +\left\{\frac{1}{2} \sum_{\mu} \sum_{\nu} \iint d x d y \frac{\delta}{\delta \psi_{\mu}(x)} \frac{\delta}{\delta \psi_{\nu}(y)} D^{\mu \nu}(x, y)\right\} P\left[\psi, \psi^{+}, \psi^{*}, \psi^{+*}\right] \\
\psi_{\mu}(x)= & \sum_{k} \alpha_{\mu k}(t) \phi_{k}^{\mu}(x, t) \quad \alpha_{\mu k}(t)=\int d y \phi_{k}^{-\mu}(y, t) \psi_{\mu}(y) \\
\frac{\partial}{\partial t} \psi_{\mu}^{s}(x, t)= & A^{\mu}(x)+\sum_{a} N_{a}^{\mu}(x) \Gamma_{a}\left(t_{+}\right) \\
D^{\mu \nu}(x, y)= & \sum_{a} N_{a}^{\mu}(x) N_{a}^{\nu}(y) \quad \alpha_{\mu k}^{s}(t)=\int d y \phi_{k}^{-\mu}(y, t) \psi_{\mu}^{s}(y, t) \\
\psi_{\mu}^{s}(x, t)= & \sum_{k} \alpha_{\mu k}^{s}(t) \phi_{k}^{\mu}(x, t) \quad(172)
\end{aligned}
$$


For the mode theory results:

$$
\begin{aligned}
\frac{\partial}{\partial t} P\left(\alpha, \alpha^{+}, \alpha^{*}, \alpha^{+*}\right) & =\left\{-\sum_{\mu k} \frac{\partial}{\partial \alpha_{\mu k}} A_{k}^{\mu}+\frac{1}{2} \sum_{\mu k} \sum_{\nu l} \frac{\partial}{\partial \alpha_{\mu k}} \frac{\partial}{\partial \alpha_{\nu l}} D_{k l}^{\mu \nu}\right\} P\left(\alpha, \alpha^{+}, \alpha^{*}, \alpha^{+*}\right) \\
\frac{\partial}{\partial t} \alpha_{\mu k}^{s} & =A_{k}^{\mu}+\sum_{l} C_{k l}^{\mu} \alpha_{\mu l}^{s}+\sum_{a} B_{k a}^{\mu} \Gamma_{a}\left(t_{+}\right) \\
D_{k l}^{\mu \nu} & =\sum_{a} B_{k a}^{\mu} B_{l a}^{\nu}
\end{aligned}
$$

Conversions between the quantities are as follows

$$
\begin{aligned}
A_{k}^{\mu} & =\int d x \phi_{k}^{-\mu}(x, t) A^{\mu}(x) \quad A^{\mu}(x)=\sum_{k} \phi_{k}^{\mu}(x, t) A_{k}^{\mu} \\
D_{k l}^{\mu \nu} & =\iint d x d y \phi_{k}^{-\mu}(x, t) D^{\mu \nu}(x, y) \phi_{l}^{-\nu}(y, t) \quad D^{\mu \nu}(x, y)=\sum_{k l} \phi_{k}^{\mu}(x, t) D_{k l}^{\mu \nu} \phi_{l}^{\nu}(y, t) \\
N_{a}^{\mu}(x) & =\sum_{k} B_{k a}^{\mu} \phi_{k}^{\mu}(x, t)
\end{aligned}
$$

Key results for the time dependences are

$$
\begin{aligned}
\frac{\partial}{\partial t} \phi_{k}^{\mu}(x, t) & =\sum_{l} C_{k l}^{-\mu} \phi_{l}^{\mu}(x, t) \\
\frac{\partial}{\partial t} \alpha_{\mu k} & =\sum_{l} C_{k l}^{\mu} \alpha_{\mu l}
\end{aligned}
$$

with coupling coefficients

$$
\begin{aligned}
C_{k l}^{-}(t) & =\int d x \frac{\partial \phi_{k}^{*}(x, t)}{\partial t} \phi_{l}(x, t)=C_{k l}(t) \\
C_{k l}^{+}(t) & =\int d x \frac{\partial \phi_{k}(x, t)}{\partial t} \phi_{l}^{*}(x, t)=C_{k l}^{*}(t)
\end{aligned}
$$




\section{References}

[1] A.J. Leggett, Rev. Mod. Phys. 73307 (2001).

[2] M. R. Andrews, C. G. Townsend, H.-J. Miesner, D. S. Durfee, D. M. Kurn and W. Ketterle, Science 275, 637 (1997).

[3] D. S. Hall, M. R. Matthews, C. E. Wieman and E. A. Cornell, Phys. Rev. Letts. 81, 1543 (1998).

[4] S. L. Cornish, N. R. Claussen, J. L. Roberts, E. A. Cornell and C. E. Wieman, Phys. Rev. Letts. 85, 1795 (2000).

[5] C. Orzel, A. K. Tuchman, M. L. Fenselau, M. Yasuda and M. A. Kasevich, Science 291, 2386 (2001).

[6] J. Esteve, C. Gross, A. Weller, S. Giovanazzi and M. K. Oberthaler, Nature, 455, 1216 (2008).

[7] P. Bouyer and M. A. Kasevich, Phys. Rev. A 56, R1083 (1997).

[8] J.A. Dunningham, K. Burnett and S.M. Barnett, Phys. Rev. Letts. 89, 150401 (2002).

[9] K. Bongs and K. Sengstock, Rep. Prog. Phys. 67, 907 (2004).

[10] J. Dunningham, K. Burnett and W. D. Phillips, Phil. Trans. R. Soc. A 363, 2165 (2005).

[11] A. D. Cronin, J. Schmeidmayer and D. E. Pritchard, Rev. Mod. Phys. 81, 1051 (2009).

[12] T. Schumm, S. Manz, R. Bucker, D. A. Smith and J. Schmiedmayer, In Atom Chips, Eds. J. Reichel and V. Vuletic (Wiley, New York, 2011).

[13] L. Pitaevskii and S. Stringari, Bose-Einstein Condensation, (Oxford University Press, Oxford, 2003).

[14] B. J. Dalton, Ann. Phys. 326, 668 (2011).

[15] R. Graham and H. Haken, Z. Phys. 234, 193 (1970).

[16] R. Graham and H. Haken, Z. Phys. 235, 166 (1970).

[17] P. D. Drummond and S. J. Carter, J. Amer. Opt. Soc. B 4, 1565 (1987).

[18] T. A. B. Kennedy and E. M. Wright, Phys. Rev. A 38212 (1988).

[19] A. Gatti, H. Wiedemann, L. A. Lugiato, I. Marzoli, G.-L. Oppo and S. M Barnett, Phys. Rev. A 56877 (1997).

[20] M. J. Steel, M. K. Olsen, L. I. Plimak, P. D. Drummond, S. M. Tan, M. J. Collett, D. F. Walls and R. Graham, Phys. Rev. A 584824 (1998). 
[21] K. Molmer, New J. Phys. 5, 55.1 (2003).

[22] C. W. Gardiner and P. Zoller, Phys. Rev. A 58536 (1998).

[23] C. W. Gardiner and P. Zoller, Phys. Rev. A 61033601 (2000).

[24] B. J. Dalton, J. Phys: Conf. Ser. 67, 012059 (2007).

[25] S. E. Hoffmann, J. F. Corney and P. D. Drummond, Phys. Rev. A 78 $013622(2008)$.

[26] P. B. Blakie, A. S. Bradley, M. J. Davis, R. J. Ballagh and C. W. Gardiner, Adv. Phys. 37, 363 (2008).

[27] M. Egorov, R. P. Anderson, V. Ivannikov, B. Opanchuk and P. D. Drummond, Phys. Rev. A 84021605 (2011).

[28] E. P. Gross, Nuo. Cim. 20, 454 (1961).

[29] L. P. Pitaevskii, Zh. Eksp. Teor. Fiz. 40, 646 (1961).

[30] P. A. M. Dirac, Proc. Cambridge. Philos. Soc. 26, 376 (1930).

[31] J. Frenkel, Wave Mechanics, (Oxford University Press, Oxford, 1934).

[32] R. W. Spekkens and J. E. Sipe, Phys. Rev. A 59, 3868 (1999).

[33] C. Menotti, J. R. Anglin, J. I. Cirac and P. Zoller, Phys. Rev. A 63, 023601 (2001).

[34] B. J. Dalton, J. Mod. Opt. 54, 615 (2007).

[35] A. I. Streltsov, O. E. Alon and L. S. Cederbaum, Phys. Rev. A 73, 063626 (2006).

[36] A. I. Streltsov, O. E. Alon and L. S. Cederbaum, Phys. Rev. Letts. 99, $030402(2007)$.

[37] O. E. Alon, A. I. Streltsov and L. S. Cederbaum, Phys. Rev. A 77, 033613 (2008).

[38] K. Sakmann, A. I. Streltsov, O. E. Alon and L. S. Cederbaum, Phys. Rev. Letts. 103, 220601 (2009).

[39] B. J. Dalton and S. Ghanbari, J. Mod. Opt. 59, 287 (2012).

[40] N. N. Bogoliubov, J. Phys. (USSR), 11, 23 (1947).

[41] R. Bach and K. Rzazewski, Phys. Rev. A 70, 063622 (2004).

[42] P. D. Drummond and C. W. Gardiner, J. Phys. A: Math. Gen. 13, 2353 (1980). 
[43] C.W. Gardiner, Quantum Noise (Springer-Verlag, Berlin 1991).

[44] S. M. Barnett and P. M. Radmore, Methods in Theoretical Quantum Optics, (Clarendon Press, Oxford, UK, 1997).

[45] T. Takagi, Collected Papers of T Takagi (Springer-Verlag, Berlin, Germany, 1990).

[46] R. A. Horn and C. R. Johnson, Matrix Analysis, (Cambridge University Press, Cambridge, UK, 1985).

\subsection{Acknowledgements}

This work was supported by the Australian Research Council Centre of Excellence for Quantum Atom Optics (ACQAO). The author thanks J. Corney, P. Deuar for helpful discussions and a referee for valuable advice on improving the presentation. . 


\section{Appendix - Functional Calculus}

\subsection{Functionals}

Essentially a functional $F[\psi(x)]$ of a field function $\psi(x)$ just defines a process that results in a c-number which depends on all the values of the field function, that is over the entire range of positions $x$. Unless otherwise stated the field function is complex. Generalisations to cases involving several functions $\psi(x), \psi^{+}(x)$ or $\psi(x), \psi^{+}(x), \psi^{*}(x), \psi^{+*}(x)$ etc are straightforward, as also when $x$ refers to position $\mathbf{r}$ in a $3 \mathrm{D}$ space. The field functions may also sometimes be time-dependent.

Examples of functionals are numerous. If $\chi(x)$ is fixed then the integral $\int d x \chi^{*}(x) \psi(x)$ is a functional of $\psi(x)$, often written $\chi[\psi(x)]$. This functional defines the scalar product, often written $(\chi, \psi)$. Here $\chi^{*}(x)$ acts as a kernel. A function $\psi(y)$ can also be considered as a functional of $\psi(x)$, with the delta function acting as the kernel. This specific functional is written as $F_{y}[\psi(x)]=$ $\int d x \delta(y-x) \psi(x)=\psi(y)$.

If the function $\psi(x)$ is expanded in terms of orthonormal mode functions $\phi_{k}(x)$ with expansion coefficients $\alpha_{k}$

$$
\begin{aligned}
\psi(x) & =\sum_{k} \alpha_{k} \phi_{k}(x) \\
\alpha_{k} & =\int d x \phi_{k}^{*}(x) \psi(x)
\end{aligned}
$$

then the functional $F[\psi(x)]$ can always be determined from the $\alpha_{k}$, so is the same as the function $f\left(\alpha_{1}, \alpha_{2}, . ., \alpha_{k}, ..\right)$ obtained by replacing $\psi(x)$ by its mode expansion in the process that determines the functional.

$$
F[\psi(x)]=f\left(\alpha_{1}, \alpha_{2}, . ., \alpha_{k}, . .\right)
$$

The orthonormality and completeness relationships are

$$
\begin{aligned}
\int d x \phi_{k}^{*}(x, t) \phi_{l}(x, t) & =\delta_{k l} \\
\sum_{k} \phi_{k}(x, t) \phi_{k}^{*}(y, t) & =\delta(x-y)
\end{aligned}
$$

\subsection{Functional Differentiation}

A summary of functional differentiation is as follows. For a functional $F[\psi(x)]$ of a field $\psi(x)$ the functional derivative $\frac{\delta F[\psi(x)]}{\delta \psi(x)}$ is defined by

$$
F[\psi(x)+\delta \psi(x)] \doteqdot F[\psi(x)]+\int d x \delta \psi(x)\left(\frac{\delta F[\psi(x)]}{\delta \psi(x)}\right)_{x}
$$


where $\delta \psi(x)$ is any small but arbitrary change in $\psi(x)$. In this equation the left side is a functional of $\psi(x)+\delta \psi(x)$ and the first term on the right side is a functional of $\psi(x)$. The second term on the right side is a functional of $\delta \psi(x)$ and thus the functional derivative must be a function of $x$; hence the subscript $x$. In most situations this subscript will be left understood. If we write $\delta \psi(x)=\epsilon \delta(x-y)$ for small $\epsilon$ then an equivalent result for the functional derivative at $x=y$ is

$$
\left(\frac{\delta F[\psi(x)]}{\delta \psi(x)}\right)_{x=y}=\lim _{\epsilon \rightarrow 0}\left(\frac{F[\psi(x)+\epsilon \delta(x-y)]-F[\psi(x)]}{\epsilon}\right) .
$$

Note that for a fixed function $\xi(x)$

$$
\frac{\delta \xi(x)}{\delta \psi(x)}=0
$$

since the fixed function does not change when $\psi(x)$ does. On the other hand

$$
\left(\frac{\delta \psi(y)}{\delta \psi(x)}\right)_{x}=\left(\frac{\delta F_{y}[\psi(x)]}{\delta \psi(x)}\right)_{x}=\delta(y-x)
$$

Functional differentiation satisfies many of the rules of ordinary differentiation including a product rule

$$
\left(\frac{\delta\{F[\psi(x)] G[\psi(x)]\}}{\delta \psi(x)}\right)=\left(\frac{\delta F[\psi(x)]}{\delta \psi(x)}\right) G[\psi(x)]+F[\psi(x)]\left(\frac{\delta G[\psi(x)]}{\delta \psi(x)}\right)
$$

Note that for a fixed function $\xi(x)$ we have from Eqs.(186) and (184)

$$
\frac{\delta\{\xi(x) F[\psi(x)]\}}{\delta \psi(x)}=\xi(x)\left(\frac{\delta F[\psi(x)]}{\delta \psi(x)}\right)
$$

so a fixed function may be moved through the functional differentiation.

Mode expansions can be used to relate functional differentiation and ordinary differentiation.

$$
\begin{aligned}
\left(\frac{\delta F[\psi(x)]}{\delta \psi(x)}\right)_{x} & =\sum_{k} \phi_{k}^{*}(x) \frac{\partial f\left(\alpha_{1}, \alpha_{2}, . ., \alpha_{k}, . .\right)}{\partial \alpha_{k}} \\
\frac{\partial f\left(\alpha_{1}, \alpha_{2}, . ., \alpha_{k}, . .\right)}{\partial \alpha_{k}} & =\int d x \phi_{k}(x)\left(\frac{\delta F[\psi(x)]}{\delta \psi(x)}\right)_{x}
\end{aligned}
$$

Note that the first of these simple results are obtained by considering in (182) the change in $\delta \psi(x)=\sum_{k} \delta \alpha_{k} \phi_{k}(x)$ given by changes in the $\alpha_{k}$, and then equating $F[\psi(x)+\delta \psi(x)]-F[\psi(x)]$ to the corresponding result $f\left(\alpha_{1}+\delta \alpha_{1}, \alpha_{2}+\right.$ $\left.\delta \alpha_{2}, . ., \alpha_{k}+\delta \alpha_{k}, ..\right)-f\left(\alpha_{1}, \alpha_{2}, . ., \alpha_{k}, ..\right)$. The second is then obtained via the orthogonality result (180). 
A Taylor series expansion to higher orders can also be obtained. Thus to second order

$F[\psi(x)+\delta \psi(x)] \doteqdot F[\psi(x)]+\int d x \delta \psi(x)\left(\frac{\delta F[\psi(x)]}{\delta \psi(x)}\right)_{x}+\frac{1}{2} \iint d x d y \delta \psi(x) \delta \psi(y)\left(\frac{\delta^{2} F[\psi(x)]}{\delta \psi(x) \delta \psi(y)}\right)_{x, y}$

and can be obtained via a Taylor expansion of $f\left(\alpha_{1}, \alpha_{2}, . ., \alpha_{k}, ..\right)$ plus the relationships in (188).

\subsection{Functional Integration}

A brief summary of functional integration is as follows. If there are $n$ modes then the range for each function $\psi(x)$ is divided up into $n$ small intervals $\Delta x_{i}=$ $x_{i+1}-x_{i}$ (the $i$ th interval, where $\epsilon>\left|\Delta x_{i}\right|$ ), then we may specify the value $\psi_{i}$ of the function $\psi(x)$ in the $i$ th interval via the average

$$
\psi_{i}=\frac{1}{\Delta x_{i}} \int_{\Delta x_{i}} d x \psi(x)
$$

and then any functional $F[\psi(x)]$ may be regarded as a function $F\left(\psi_{1}, \psi_{2}, . ., \psi_{i}, . ., \psi_{n}\right)$

of all the $\psi_{i}$, and ordinary integration over the $\psi_{i}$ is used to define the functional integral. If each function $\psi(x)=\psi_{x}(x)+i \psi_{y}(x)$.is written in terms of its real and imaginary parts, then the functional integration becomes an ordinary integration over the values $\psi_{i x}, \psi_{i y}$ of these components in each interval $i$ of the function $F\left(\psi_{1}, \psi_{2}, . ., \psi_{i}, . ., \psi_{n}\right)$ multiplied by a suitably chosen weight function $w\left(\psi_{1}, \psi_{2}, . ., \psi_{i}, . ., \psi_{n}\right)$. Thus the functional integral is defined by

$$
\begin{aligned}
\int D^{2} \psi F[\psi(x)]= & \lim _{n \rightarrow \infty} \lim _{\epsilon \rightarrow 0} \int \ldots \int d^{2} \psi_{1} d^{2} \psi_{2} . . d^{2} \psi_{i} . . d^{2} \psi_{n} w\left(\psi_{1}, \psi_{2}, . ., \psi_{i}, . ., \psi_{n}\right) \\
& \times F\left(\psi_{1}, \psi_{2}, . ., \psi_{i}, . ., \psi_{n}\right)
\end{aligned}
$$

where the number of modes is increased to infinity along with the space interval decreasing to zero. The symbol $D^{2} \psi$ stands for $d^{2} \psi_{1} d^{2} \psi_{2} . . d^{2} \psi_{i} . . d^{2} \psi_{n} w\left(\psi_{1}, \psi_{2}, . ., \psi_{i}, . ., \psi_{n}\right)$, where the quantity $d^{2} \psi_{i}$ means $d \psi_{i x} d \psi_{i y}$.

A useful integration by parts rule can often be established from Eq.(186). Consider the functional $H[\psi(x)]=F[\psi(x)] G[\psi(x)]$. Then

$$
\int D^{2} \psi F[\psi(x)]\left(\frac{\delta G[\psi(x)]}{\delta \psi(x)}\right)=\int D^{2} \psi\left(\frac{\delta H[\psi(x)]}{\delta \psi(x)}\right)-\int D^{2} \psi\left(\frac{\delta F[\psi(x)]}{\delta \psi(x)}\right) G[\psi(x)]
$$

Functional integration and phase space integration are inter-related via the mode expansion, so we can relate the value $\psi_{i}$ of the function in the $i$ th interval to that of the mode function $\phi_{k i}$ via

$$
\psi_{i}=\sum_{k} \alpha_{k} \phi_{k i}
$$


Choosing the number of intervals to coincide with the number of modes the inte-

gration $\int \ldots \int d^{2} \psi_{1} d^{2} \psi_{2} . . d^{2} \psi_{i} . . d^{2} \psi_{n}$ can be changed to an integration $\int \ldots \int d^{2} \alpha_{1} d^{2} \alpha_{2} . . d^{2} \alpha_{k} . . d^{2} \alpha_{n}$ over the expansion coefficients $\alpha_{1}, \alpha_{2}, . ., \alpha_{k}, . . \alpha_{n}$. We have

$$
\begin{aligned}
\int D^{2} \psi F[\psi(x)]= & \lim _{n \rightarrow \infty} \lim _{\epsilon \rightarrow 0} \int \ldots \int d^{2} \alpha_{1} d^{2} \alpha_{2} . . d^{2} \alpha_{k} . . d^{2} \alpha_{n}\left\|J\left(\alpha_{1}, \alpha_{2}, . ., \alpha_{k}, . . \alpha_{n}\right)\right\| \\
& \times v\left(\alpha_{1}, \alpha_{2}, . ., \alpha_{k}, . . \alpha_{n}\right) f\left(\alpha_{1}, \alpha_{2}, . ., \alpha_{k}, . . \alpha_{n}\right)
\end{aligned}
$$

where $v\left(\alpha_{1}, \alpha_{2}, . ., \alpha_{k}, . . \alpha_{n}\right)$ is the function that equals $w\left(\psi_{1}, \psi_{2}, . ., \psi_{i}, . ., \psi_{n}\right)$ and the Jacobian is given by

$$
\left\|J\left(\alpha_{1}, \alpha_{2}, . ., \alpha_{k}, . . \alpha_{n}\right)\right\|=\left\|\begin{array}{cccccccc}
\frac{\partial \psi_{1 x}}{\partial \alpha_{1 x}} & \frac{\partial \psi_{1 x}}{\partial \alpha_{2 x}} & \ldots & \frac{\partial \psi_{1 x}}{\partial \alpha_{n x}} & \frac{\partial \psi_{1 x}}{\partial \alpha_{1 y}} & \frac{\partial \psi_{1 x}}{\partial \alpha_{2 y}} & \ldots & \frac{\partial \psi_{1 x}}{\partial \alpha_{n y}} \\
\frac{\partial \psi_{2 x}}{\partial \alpha_{1 x}} & \frac{\partial \psi_{2 x}}{\partial \alpha_{2 x}} & \ldots & \frac{\partial \psi_{2 x}}{\partial \alpha_{n x}} & \frac{\partial \psi_{2 x}}{\partial \alpha_{1 y}} & \frac{\partial \psi_{2 x}}{\partial \alpha_{2 y}} & \ldots & \frac{\partial \psi_{2 x}}{\partial \alpha_{n y}} \\
\ldots & \ldots & \ldots & \ldots & \ldots & \ldots & \ldots & \ldots \\
\frac{\partial \psi_{n x}}{\partial \alpha_{1 x}} & \frac{\partial \psi_{n x}}{\partial \alpha_{2 x}} & & \frac{\partial \psi_{n x}}{\partial \alpha_{n x}} & \frac{\partial \psi_{n x}}{\partial \alpha_{1 y}} & \frac{\partial \psi_{n x}}{\partial \alpha_{2 y}} & & \frac{\partial \psi_{n x}}{\partial \alpha_{n y}} \\
\frac{\partial \psi_{1 y}}{\partial \alpha_{1 x}} & \frac{\partial \psi_{1 y}}{\partial \alpha_{2 x}} & \ldots & \frac{\partial \psi_{1 y}}{\partial \alpha_{n x}} & \frac{\partial \psi_{1 y}}{\partial \alpha_{1 y}} & \frac{\partial \psi_{1 y}}{\partial \alpha_{2 y}} & \ldots & \frac{\partial \psi_{1 y}}{\partial \alpha_{n y}} \\
\frac{\partial \psi_{2 y}}{\partial \alpha_{1 x}} & \frac{\partial \psi_{2 y}}{\partial \alpha_{2 x}} & \ldots & \frac{\partial \psi_{2 y}}{\partial \alpha_{n x}} & \frac{\partial \psi_{2 y}}{\partial \alpha_{1 y}} & \frac{\partial \psi_{2 y}}{\partial \alpha_{2 y}} & \ldots & \frac{\partial \psi_{2 y}}{\partial \alpha_{n y}} \\
\ldots & \ldots & \ldots & \ldots & \ldots & \ldots & \ldots & \ldots \\
\frac{\partial \psi_{n y}}{\partial \alpha_{1 x}} & \frac{\partial \psi_{n y}}{\partial \alpha_{2 x}} & & \frac{\partial \psi_{n y}}{\partial \alpha_{n x}} & \frac{\partial \psi_{n y}}{\partial \alpha_{1 y}} & \frac{\partial \psi_{n y}}{\partial \alpha_{2 y}} & & \frac{\partial \psi_{n y}}{\partial \alpha_{n y}}
\end{array}\right\|
$$

Now using Eq.(193)

$$
\begin{array}{lll}
\frac{\partial \psi_{i x}}{\partial \alpha_{k x}}=\phi_{k i x} & & \frac{\partial \psi_{i x}}{\partial \alpha_{k y}}=-\phi_{k i y} \\
\frac{\partial \psi_{i y}}{\partial \alpha_{k x}}=\phi_{k i y} & \frac{\partial \psi_{i y}}{\partial \alpha_{k y}}=\phi_{k i x}
\end{array}
$$

and evaluating the Jacobian after showing that $\left(J J^{T}\right)_{i a k b}=\delta_{i k} \delta_{a b} / \Delta x_{i}$ using the completeness relationship in Eq. (181) we find that

$$
\left\|J\left(\alpha_{1}, \alpha_{2}, . ., \alpha_{k}, . . \alpha_{n}\right)\right\|=\prod_{i} \frac{1}{\left(\Delta x_{i}\right)}
$$

and thus

$$
\begin{aligned}
\int D^{2} \psi F[\psi(x)]= & \lim _{n \rightarrow \infty} \lim _{\epsilon \rightarrow 0} \int \ldots \int d^{2} \alpha_{1} d^{2} \alpha_{2} . . d^{2} \alpha_{k} . . d^{2} \alpha_{n} \prod_{i} \frac{1}{\left(\Delta x_{i}\right)} \\
& \times v\left(\alpha_{1}, \alpha_{2}, . ., \alpha_{k}, . . \alpha_{n}\right) f\left(\alpha_{1}, \alpha_{2}, . ., \alpha_{k}, . . \alpha_{n}\right)
\end{aligned}
$$

This key result expresses the original functional integral as a phase space integral over the expansion coefficients $\alpha_{k}$ of the function $\psi(x)$ in terms of the mode functions $\phi_{k}(x)$.

The general result can be simplified with a special choice of the weight function

$$
w\left(\psi_{1}, \psi_{2}, . ., \psi_{i}, . ., \psi_{n}\right)=\prod_{i}\left(\Delta x_{i}\right)
$$


and we then get a simple expression for the functional integral

$$
\int D^{2} \psi F[\psi(x)]=\lim _{n \rightarrow \infty} \lim _{\epsilon \rightarrow 0} \int \ldots \int d^{2} \alpha_{1} d^{2} \alpha_{2} . . d^{2} \alpha_{k} . . d^{2} \alpha_{n} f\left(\alpha_{1}, \alpha_{2}, . ., \alpha_{k}, . . \alpha_{n}\right)
$$

In this form of the functional integral the original functional $F[\psi(x)]$ has been replaced by the equivalent function $f\left(\alpha_{1}, \alpha_{2}, . ., \alpha_{k}, . . \alpha_{n}\right)$ of the expansion coefficients $\alpha_{k}$, and the functional integration is now replaced by a phase space integration over the expansion coefficients. 


\section{Appendix - Equivalence of Separate Mode and Field Theory Treatments}

\subsection{Fokker-Planck and Functional Fokker-Planck Equa- tions}

The functional Fokker-Planck equation for the distribution functional $P\left[\underset{\psi}{\psi}, \underset{\psi^{*}}{\rightarrow}\right]$ must be equivalent to the ordinary Fokker-Planck equation Eq. (63) for the distribution function $P\left(\alpha, \alpha^{+}, \alpha^{*}, \alpha^{+*}\right)$. The drift and diffusion terms can be interrelated because functional and ordinary differentiation are related, as explained in Appendix 6. We use

$$
\begin{aligned}
\left(\frac{\delta}{\delta \psi_{A}^{\mu}(x, t)}\right)_{x} & =\sum_{k} \phi_{A k}^{-\mu}(x, t) \frac{\partial}{\partial \alpha_{A \mu k}(t)} \\
\frac{\partial}{\partial \alpha_{A \mu k}(t)} & =\int d x \phi_{A k}^{\mu}(x, t)\left(\frac{\delta}{\delta \psi_{A}^{\mu}(x, t)}\right)_{x}
\end{aligned}
$$

in the functional Fokker-Planck equation (129) which becomes

$$
\begin{aligned}
& \frac{\partial}{\partial t} P\left[\underset{\psi}{\rightarrow}, \underline{\psi}^{*}\right] \\
= & \frac{\partial}{\partial t} P\left(\alpha, \alpha^{+}, \alpha^{*}, \alpha^{+*}\right) \\
= & \left\{-\sum_{\mu A} \int d x \sum_{k} \phi_{A k}^{-\mu}(x, t) \frac{\partial}{\partial \alpha_{\mu A k}} A_{A}^{\mu}(x)\right\} P\left(\alpha, \alpha^{+}, \alpha^{*}, \alpha^{+*}\right) \\
& +\left\{\frac{1}{2} \sum_{\mu A} \sum_{\nu B} \iint d x d y \sum_{k} \phi_{A k}^{-\mu}(x, t) \frac{\partial}{\partial \alpha_{\mu A k}} \sum_{l} \phi_{B l}^{-\nu}(y, t) \frac{\partial}{\partial \alpha_{\nu B l}} E_{A B}^{\mu \nu}(x, y)\right\} P\left(\alpha, \alpha^{+}, \alpha^{*}, \alpha^{+*}\right) \\
= & \left\{-\sum_{\mu A k} \frac{\partial}{\partial \alpha_{\mu A k}} A_{A k}^{\mu}+\frac{1}{2} \sum_{\mu A k} \sum_{\nu B l} \frac{\partial}{\partial \alpha_{\mu A k}} \frac{\partial}{\partial \alpha_{\nu B l}} E_{A k B l}^{\mu \nu}\right\} P\left(\alpha, \alpha^{+}, \alpha^{*}, \alpha^{+*}\right)
\end{aligned}
$$

and gives the Fokker-Planck equation (63) with

$$
\begin{aligned}
A_{A k}^{\mu} & =\int d x \phi_{A k}^{-\mu}(x, t) A_{A}^{\mu}(x) \\
A_{A}^{\mu}(x) & =\sum_{k} \phi_{A k}^{\mu}(x, t) A_{A k}^{\mu} \\
E_{A k B l}^{\mu \nu} & =\iint d x d y \phi_{A k}^{-\mu}(x, t) E_{A B}^{\mu \nu}(x, y) \phi_{B l}^{-\nu}(y, t) \\
E_{A B}^{\mu \nu}(x, y) & =\sum_{k l} \phi_{A k}^{\mu}(x, t) E_{A k B l}^{\mu \nu} \phi_{B l}^{\nu}(y, t)
\end{aligned}
$$


which is the same as Eqs. (63). Note that in particular

$$
\begin{aligned}
& \iint d x d y \phi_{A k}^{-\mu}(x, t) \frac{1}{2}\left\{\delta_{A C} \delta_{B N C} \delta_{\mu-\nu}\left(\sum_{m} \sum_{n} \phi_{A m}^{\mu}(x, t) C_{A m B n}^{\mu}(t) \phi_{B n}^{\nu}(y, t)\right)\right\} \phi_{B l}^{-\nu}(y, t) \\
&+\iint d x d y \phi_{A k}^{-\mu}(x, t) \frac{1}{2}\left\{\delta_{B C} \delta_{A N C} \delta_{\nu-\mu}\left(\sum_{m} \sum_{n} \phi_{B n}^{\nu}(y, t) C_{B n A m}^{\nu}(t) \phi_{A m}^{\mu}(x, t)\right)\right\} \phi_{B l}^{-\nu}(y, t) \\
&=\frac{1}{2}\left\{\delta_{A C} \delta_{B N C} \delta_{\mu-\nu} C_{A k B l}^{\mu}+\delta_{B C} \delta_{A N C} \delta_{\nu-\mu} C_{B l A k}^{\nu}\right\}
\end{aligned}
$$

as in Eq. (64). Thus the functional and ordinary Fokker-Planck equations for the hybrid case are consistent with each other.

\subsection{Ito Stochastic Equations for Fields and Phase Vari- ables}

To see how the stochastic fields $\psi_{A}^{\mu s}(x, t)$ are related to the stochastic phases $\alpha_{\mu A k}^{s}$, we find that the Ito stochastic field equation (139) becomes on substituting for $\mathcal{G}^{\mu}(x)$ and $\mathcal{N}_{a}^{\mu}(x)$ from (147), (150) and (156)

$$
\begin{aligned}
\delta \psi_{A}^{\mu s}(x, t)= & \left(\sum_{k} \phi_{A k}^{\mu}(x, t) A_{A k}^{\mu}+\sum_{k} \phi_{A k}^{\mu}(x, t) \sum_{B l} C_{A k B l}^{\mu} \alpha_{\mu B l}^{s}+\sum_{k} \frac{\partial}{\partial t} \phi_{A k}^{\mu}(x, t) \alpha_{\mu A k}^{s}\right) \delta t \\
& +\sum_{k a} K_{A k a}^{\mu} \phi_{A k}^{\mu}(x, t) \int_{t}^{t+\delta t} d t_{1} \Gamma_{a}\left(t_{1}\right) \\
= & \sum_{k} \phi_{A k}^{\mu}(x, t)\left\{A_{A k}^{\mu} \delta t+\sum_{B l} C_{A k B l}^{\mu} \alpha_{\mu B l}^{s} \delta t+\sum_{a} K_{A k a}^{\mu} \int_{t}^{t+\delta t} d t_{1} \Gamma_{a}\left(t_{1}\right)\right\} \\
& +\sum_{k} \frac{\partial}{\partial t} \phi_{A k}^{\mu}(x, t) \alpha_{\mu A k}^{s} \delta t \\
= & \sum_{k} \phi_{A k}^{\mu}(x, t) \delta \alpha_{\mu A k}^{s}+\sum_{k} \frac{\partial}{\partial t} \phi_{A k}^{\mu}(x, t) \alpha_{\mu A k}^{s} \delta t
\end{aligned}
$$

on substituting for $\delta \alpha_{\mu A k}^{s}$ from Eq.(87)

$$
\frac{\partial}{\partial t} \alpha_{\mu A k}^{s}=A_{A k}^{\mu}+\sum_{l} C_{A k B l}^{\mu} \alpha_{B \mu l}^{s}+\sum_{a} K_{A k a}^{\mu} \Gamma_{a}\left(t_{+}\right)
$$

and substituting from (60) for the time derivative of the mode function. Hence we see that the Ito stochastic equation for $\psi_{A}^{\mu s}(x, t)$ is

$$
\frac{\partial}{\partial t} \psi_{A}^{\mu s}(x, t)=\sum_{k} \frac{\partial}{\partial t} \alpha_{\mu A k}^{s}(t) \phi_{A k}^{\mu}(x, t)+\sum_{k} \alpha_{\mu A k}^{s} \frac{\partial}{\partial t} \phi_{A k}^{\mu}(x, t)
$$

This shows that the stochastic fields are given by

$$
\psi_{A}^{\mu s}(x, t)=\sum_{k} \alpha_{\mu A k}^{s}(t) \phi_{A k}^{\mu}(x, t)
$$


where the stochastic feature results only from the phase variables being replaced by stochastic variables. These equations show that the stochastic field equation for $\psi_{\mu}^{s}(x, t)$ is equivalent to the stochastic phase variable equation for the $\alpha_{\mu k}^{s}(t)$. 


\section{Appendix - Derivation of Langevin Equations}

Details in carrying out the stochastic averaging for the derivation of the Langevin equations in Section 2 are set out here.

For the first order derivative terms

$$
\begin{aligned}
& \frac{\left\{\sum_{\mu A k}\left[\frac{\partial}{\partial \alpha_{\mu A k}} F\left(\alpha, \alpha^{+}\right)\right] \delta \alpha_{\mu A k}^{s}(t)\right\}}{\sum_{\mu A k}\left[\frac{\partial}{\partial \alpha_{\mu A k}} F\left(\alpha, \alpha^{+}\right)\right] \mathcal{A}_{A k}^{\mu}\left(\alpha_{\xi D m}^{s}(t)\right)} \delta t \\
& +\frac{\sum_{\mu A k}\left[\frac{\partial}{\partial \alpha_{\mu A k}} F\left(\alpha, \alpha^{+}\right)\right] \sum_{a} \mathcal{B}_{A k a}^{\mu}\left(\alpha_{\xi D m}^{s}(t)\right)}{\sum_{t}^{t+\delta t} d t_{1} \Gamma_{a}\left(t_{1}\right)} \\
= & \int_{\mu A k}\left[\frac{\partial}{\partial \alpha_{\mu A k}} F\left(\alpha, \alpha^{+}\right)\right] \mathcal{A}_{A k}^{\mu}\left(\alpha_{\xi D m}^{s}(t)\right) \delta t
\end{aligned}
$$

where the stochastic average rules for sums and products have been used, the non-correlation between the averages of functions of $\alpha_{\mu A k}^{s}(t)$ at time $t$ and the $\Gamma$ at later times between $t$ to $t+\delta t$ is applied and the term involving $\overline{\Gamma_{a}\left(t_{1}\right)}$ is equal to zero from (78).

For the second order derivative terms, we have on expanding the product $\delta \alpha_{\mu A k}^{s}(t) \delta \alpha_{\nu B l}^{s}(t)$ and using the stochastic average of a sum being the same as the sum of stochastic averages

$$
\begin{aligned}
& \frac{\left\{\frac{1}{2} \sum_{\mu A k} \sum_{\nu B l}\left[\frac{\partial}{\partial \alpha_{\mu A k}} \frac{\partial}{\partial \alpha_{\nu B l}} F\left(\alpha, \alpha^{+}\right)\right] \delta \alpha_{\mu A k}^{s}(t) \delta \alpha_{\nu B l}^{s}(t)\right\}}{\frac{1}{2} \sum_{\mu A k} \sum_{\nu B l}\left[\frac{\partial}{\partial \alpha_{\mu A k}} \frac{\partial}{\partial \alpha_{\nu B l}} F\left(\alpha, \alpha^{+}\right)\right]} \\
= & \times\left[\mathcal{A}_{A k}^{\mu}\left(\alpha_{\xi D m}^{s}(t)\right) \delta t \mathcal{A}_{B l}^{\nu}\left(\alpha_{\xi D m}^{s}(t)\right) \delta t\right] \\
+ & \times \frac{1}{2} \sum_{\mu A k} \sum_{\nu B l}\left[\frac{\partial}{\partial \alpha_{\mu A k}} \frac{\partial}{\partial \alpha_{\nu B l}} F\left(\alpha, \alpha^{+}\right)\right] \\
+ & \times\left[\mathcal{A}_{A k}^{\mu}\left(\alpha_{\xi D m}^{s}(t)\right) \delta t \sum_{b} \mathcal{B}_{B l b}^{\nu}\left(\alpha_{\xi D m}^{s}(t)\right) \int_{t}^{t+\delta t} d t_{2} \Gamma_{b}\left(t_{2}\right)\right] \\
+ & \left.\times \frac{1}{2} \sum_{\mu k A}^{\mu}\left(\alpha_{\xi D m}^{s}(t)\right) \int_{t}^{t+\delta t} d t_{1} \Gamma_{a}\left(t_{1}\right) \mathcal{A}_{B l}^{\nu}\left(\alpha_{\xi D m}^{s}(t)\right) \delta t\right] \\
& \times\left[\sum_{a} \mathcal{B}_{A k a}^{\mu}\left(\alpha_{\xi D m}^{s}(t)\right) \int_{t}^{t+\delta t} d t_{1} \Gamma_{a}\left(t_{1}\right) \sum_{b} \mathcal{B}_{B l b}^{\nu}\left(\alpha_{\xi D m}^{s}(t)\right) \int_{t}^{t+\delta t} d t_{2} \Gamma_{b}\left(t_{2}\right)\right]
\end{aligned}
$$

Using the result that the stochastic averages for the functions of the $\alpha_{\xi D m}^{s}(t)$ 
and the $\Gamma_{a}\left(t_{+}\right)$are uncorrelated we find that

$$
\begin{aligned}
& \frac{\left\{\frac{1}{2} \sum_{\mu A k} \sum_{\nu B l}\left[\frac{\partial}{\partial \alpha_{\mu A k}} \frac{\partial}{\partial \alpha_{\nu B l}} F\left(\alpha, \alpha^{+}\right)\right] \delta \alpha_{\mu A k}^{s}(t) \delta \alpha_{\nu B l}^{s}(t)\right\}}{\frac{1}{2} \sum_{\mu A k} \sum_{\nu B l}\left[\frac{\partial}{\partial \alpha_{\mu A k}} \frac{\partial}{\partial \alpha_{\nu B l}} F\left(\alpha, \alpha^{+}\right)\right]\left[\mathcal{A}_{A k}^{\mu}\left(\alpha_{\xi D m}^{s}(t)\right) \mathcal{A}_{B l}^{\nu}\left(\alpha_{\xi D m}^{s}(t)\right)\right]} \delta t^{2} \\
+ & \frac{1}{2} \sum_{\mu A k} \sum_{\nu B l}\left[\frac{\partial}{\partial \alpha_{\mu A k}} \frac{\partial}{\partial \alpha_{\nu B l}} F\left(\alpha, \alpha^{+}\right)\right]\left[\mathcal{A}_{A k}^{\mu}\left(\alpha_{\xi D m}^{s}(t)\right) \delta t \sum_{b} \mathcal{B}_{B l b}^{\nu}\left(\alpha_{\xi D m}^{s}(t)\right)\right] \\
& \times \frac{\int_{t}^{t+\delta t} d t_{2} \Gamma_{b}\left(t_{2}\right)}{\delta t} \\
& \frac{1}{2} \sum_{\mu A k} \sum_{\nu B l}\left[\frac{\partial}{\partial \alpha_{\mu A k}} \frac{\partial}{\partial \alpha_{\nu B l}} F\left(\alpha, \alpha^{+}\right)\right]\left[\sum_{a} \mathcal{B}_{A k a}^{\mu}\left(\alpha_{\xi D m}^{s}(t)\right) \mathcal{A}_{B l}^{\nu}\left(\alpha_{\xi D m}^{s}(t)\right)\right] \\
& \times \int_{t}^{t+\delta t} d t_{1} \Gamma_{a}\left(t_{1}\right) \delta t \\
& +\frac{1}{2} \sum_{\mu A k} \sum_{\nu B l}\left[\frac{\partial}{\partial \alpha_{\mu A k}} \frac{\partial}{\partial \alpha_{\nu B l}} F\left(\alpha, \alpha^{+}\right)\right]\left[\sum_{a} \mathcal{B}_{A k a}^{\mu}\left(\alpha_{\xi D m}^{s}(t)\right) \sum_{b} \mathcal{B}_{B l b}^{\nu}\left(\alpha_{\xi D m}^{s}(t)\right)\right] \\
& \times \int_{t}^{t+\delta t} d t_{1} \Gamma_{a}\left(t_{1}\right) \int_{t}^{t+\delta t} d t_{2} \Gamma_{b}\left(t_{2}\right)
\end{aligned}
$$

Now the terms involving a single $\Gamma$ have a zero stochastic average, whilst the terms with two $\Gamma$ give a stochastic average proportional to $\delta t$

$$
\begin{aligned}
\overline{\int_{t}^{t+\delta t} d t_{1} \Gamma_{a}\left(t_{1}\right) \int_{t}^{t+\delta t} d t_{2} \Gamma_{b}\left(t_{2}\right)} & =\int_{t}^{t+\delta t} d t_{1} \int_{t}^{t+\delta t} d t_{2} \overline{\Gamma_{a}\left(t_{1}\right) \Gamma_{b}\left(t_{2}\right)} \\
& =\int_{t}^{t+\delta t} d t_{1} \int_{t}^{t+\delta t} d t_{2} \delta_{a b} \delta\left(t_{1}-t_{2}\right) \\
& =\delta_{a b} \delta t
\end{aligned}
$$

so that correct to order $\delta t$ the second order derivative term is

$$
\begin{aligned}
& \overline{\left\{\frac{1}{2} \sum_{\mu A k} \sum_{\nu B l}\left[\frac{\partial}{\partial \alpha_{\mu A k}} \frac{\partial}{\partial \alpha_{\nu B l}} F\left(\alpha, \alpha^{+}\right)\right] \delta \alpha_{\mu A k}^{s}(t) \delta \alpha_{\nu B l}^{s}(t)\right\}} \\
& =\overline{\frac{1}{2} \sum_{\mu A k} \sum_{\nu B l}\left[\frac{\partial}{\partial \alpha_{\mu A k}} \frac{\partial}{\partial \alpha_{\nu B l}} F\left(\alpha, \alpha^{+}\right)\right]\left[\sum_{a} \mathcal{B}_{A k a}^{\mu}\left(\alpha_{\xi D m}^{s}(t)\right) \mathcal{B}_{B l a}^{\nu}\left(\alpha_{\xi D m}^{s}(t)\right)\right]} \delta t \\
& =\overline{\frac{1}{2} \sum_{\mu A k} \sum_{\nu B l}\left[\frac{\partial}{\partial \alpha_{\mu A k}} \frac{\partial}{\partial \alpha_{\nu B l}} F\left(\alpha, \alpha^{+}\right)\right]\left[\left[\mathcal{B}\left(\alpha_{\xi D m}^{s}(t)\right) \mathcal{B}^{T}\left(\alpha_{\xi D m}^{s}(t)\right)\right]_{A k, B l}^{\mu, \nu}\right]} \delta t
\end{aligned}
$$


The remaining terms give stochastic averages correct to order $\delta t^{2}$ or higher so that we have correct to first order in $\delta t$

$$
\begin{aligned}
& \overline{F\left(\alpha^{s}(t+\delta t), \alpha^{s+}(t+\delta t)\right)}-\overline{F\left(\alpha^{s}(t), \alpha^{s+}(t)\right)} \\
& =\left\{\overline{\sum_{\mu A k}\left[\frac{\partial}{\partial \alpha_{\mu A k}} F\left(\alpha, \alpha^{+}\right)\right] \mathcal{A}_{A k}^{\mu}\left(\alpha_{\xi D m}^{s}(t)\right)}\right\} \delta t \\
& +\left\{\overline{\frac{1}{2} \sum_{\mu A k} \sum_{\nu B l}\left[\frac{\partial}{\partial \alpha_{\mu A k}} \frac{\partial}{\partial \alpha_{\nu B l}} F\left(\alpha, \alpha^{+}\right)\right]\left[\left[\mathcal{B}\left(\alpha_{\xi D m}^{s}(t)\right) \mathcal{B}^{T}\left(\alpha_{\xi D m}^{s}(t)\right)\right]_{A k, B l}^{\mu, \nu}\right]}\right\} \delta t
\end{aligned}
$$




\section{Appendix - Derivation of Functional Fokker- Planck Equation}

This Appendix contains details in the derivation of the functional Fokker-Planck equation, the final form of which is set out in Section 3 .

\subsection{Form of Characteristic Functional}

In deriving (108) we note that integrals involving cross terms between condensate and non-condensate fields are zero via mode orthogonality and hence

$$
\begin{aligned}
\int d x \hat{\Psi}(x) \Xi^{+}(x) & =\int d x \hat{\Psi}_{C}(x, t) \Xi_{C}^{+}(x, t)+\int d x \hat{\Psi}_{N C}(x, t) \Xi_{N C}^{+}(x, t) \\
& =\sum_{k \epsilon C, N C} \hat{a}_{k} \xi_{k}^{+} \\
\int d x \Xi(x) \hat{\Psi}^{\dagger}(x) & =\int d x \Xi_{N C}(x, t) \hat{\Psi}_{N C}^{\dagger}(x, t)+\int d x \Xi_{N C}(x, t) \hat{\Psi}_{N C}^{\dagger}(x, t) \\
& =\sum_{k \epsilon C, N C} \xi_{k} \hat{a}_{k}^{\dagger} \\
\left.\frac{1}{2} \int d x \Xi_{C}(x) \Xi_{C}^{+}(x)\right) & =\frac{1}{2} \sum_{k \in C} \xi_{k} \xi_{k}^{+}
\end{aligned}
$$

In deriving (113) we note that

$$
\begin{aligned}
\int d x\left(\psi_{C}(x, t) \Xi_{C}^{+}(x, t)+\Xi_{C}(x, t) \psi_{C}^{+}(x, t)\right) & =\sum_{k \in C}\left(\alpha_{k} \xi_{k}^{+}+\xi_{k} \alpha_{k}^{+}\right) \\
\int d x \psi_{N C}(x, t) \Xi_{N C}^{+}(x, t) & =\sum_{k \in N C} \alpha_{k} \xi_{k}^{+} \int d x \Xi_{N C}(x, t) \psi_{N C}^{+}(x, t)=\sum_{k \in N C} \xi_{k} \alpha_{k}^{+}
\end{aligned}
$$

\subsection{Time Derivative of Characteristic Functional}

Details for the derivation of (121) are as follows.

The time derivative in Eq.(120) can be evaluated using Eqs. (103), (14) and 
(41)

$$
\begin{aligned}
& -\frac{1}{2} \frac{\partial}{\partial t} \int d x \Xi_{C}(x, t) \Xi_{C}^{+}(x, t) \\
= & -\frac{1}{2} \int d x\left(\frac{\partial}{\partial t} \Xi_{C}(x, t) \Xi_{C}^{+}(x, t)+\Xi_{C}(x, t) \frac{\partial}{\partial t} \Xi_{C}^{+}(x, t)\right) \\
= & -\frac{1}{2} \int d x\left(\left\{\sum_{k \in C} \sum_{l \in N C} C_{k l}(t) \xi_{l}(t) \phi_{k}(x, t)+\sum_{k \in C} \sum_{l \in N C} \xi_{k}(t) C_{k l}^{*} \phi_{l}(x, t)\right\}\right. \\
& \left.\times \sum_{m \in C} \xi_{m}^{+}(t) \phi_{m}^{*}(x, t)\right) \\
& -\frac{1}{2} \int d x\left(\sum_{m \in C} \xi_{m}(t) \phi_{m}(x, t)\right. \\
& \left.\times\left\{\sum_{k \epsilon C} \sum_{l \in N C} C_{k l}^{*}(t) \xi_{l}^{+}(t) \phi_{k}^{*}(x, t)+\sum_{k \in C} \sum_{l \in N C} \xi_{k}^{+}(t) C_{k l} \phi_{l}^{*}(x, t)\right\}\right) \\
= & -\frac{1}{2}\left\{\sum_{k \epsilon C} \sum_{l \in N C} C_{k l}(t) \xi_{l}(t) \xi_{k}^{+}(t)\right\}-\frac{1}{2}\left\{\sum_{k \in C} \sum_{l \in N C} C_{k l}^{*}(t) \xi_{k}(t) \xi_{l}^{+}(t)\right\}(216)
\end{aligned}
$$

where the $l \epsilon C$ terms have been eliminated using $C_{k l}(t)+C_{l k}^{*}(t)=0$ and orthogonality of the modes for $l \epsilon N C$ and $m \epsilon C$ has been used to eliminate the second and fourth contributions.

Since for $l \epsilon N C$ and $k \epsilon C$ we have from (103)

$$
\begin{array}{rlrl}
\xi_{l}(t) & =\int d x \phi_{l}^{*}(x, t) \Xi_{N C}(x, t) & \xi_{l}^{+}(t)=\int d x \phi_{l}(x, t) \Xi_{N C}^{+}(x, t) \\
\xi_{k}(t)=\int d y \phi_{k}^{*}(y, t) \Xi_{C}(y, t) & \xi_{k}^{+}(t)=\int d y \phi_{k}(y, t) \Xi_{C}^{+}(y, t)
\end{array}
$$

we see that

$$
\begin{aligned}
& -\frac{1}{2} \frac{\partial}{\partial t} \int d x \Xi_{C}(x, t) \Xi_{C}^{+}(x, t) \\
= & \frac{1}{2}\left\{\iint d x d y\left\{\sum_{k \in C} \sum_{l \in N C} \phi_{l}^{*}(x, t) C_{k l}(t) \phi_{k}(y, t)\right\}\left(i \Xi_{N C}(x, t)\right)\left(i \Xi_{C}^{+}(y, t)\right)\right\} \\
& +\frac{1}{2}\left\{\iint d x d y\left\{\sum_{l \in C} \sum_{k \in N C} \phi_{l}^{*}(x, t) C_{l k}^{*}(t) \phi_{k}(y, t)\right\}\left(i \Xi_{C}(x, t)\right)\left(i \Xi_{N C}^{+}(y, t)\right)\right\}
\end{aligned}
$$

The exponential factor in Eq. (120) can be recombined via (107) with the normally ordered characteristic functional $\operatorname{Tr}\left\{\exp i \int d x\left(\hat{\Psi}(x) \Xi^{+}(x)\right)\right\} \hat{\rho}\left\{\exp i \int d x\left(\Xi(x) \hat{\Psi}^{\dagger}(x)\right)\right\}$ to produce the original characteristic functional $\chi\left[\Xi_{C}, \Xi_{C}^{+}, \Xi_{N C}, \Xi_{N C}^{+}\right]$. Writing 
the characteristic functional in the form in Eq. (113) and noting that multiplication by $\left(i \Xi_{N C}(x, t)\right)\left(i \Xi_{C}^{+}(y, t)\right)$ etc. can be replaced by functional differentiations such as $\frac{\delta}{\delta \psi_{N C}^{+}(x, t)} \frac{\delta}{\delta \psi_{C}(y, t)}$ we see that the second term in Eq. (119) is the sum of two contributions

$$
\begin{aligned}
& \frac{1}{2}\left\{\iint d x d y\left\{\sum_{k \in C} \sum_{l \in N C} \phi_{l}^{*}(x, t) C_{k l}(t) \phi_{k}(y, t)\right\}\left(i \Xi_{N C}(x, t)\right)\left(i \Xi_{C}^{+}(y, t)\right)\right\} \\
& \times \chi\left[\Xi_{C}, \Xi_{C}^{+}, \Xi_{N C}, \Xi_{N C}^{+}\right] \\
& =\int D^{2} \underline{\psi} \frac{1}{2} \iint d x d y\left\{\sum_{k \in C} \sum_{l \in N C} \phi_{l}^{*}(x, t) C_{k l}(t) \phi_{k}(y, t)\right\}\left\{\frac{\delta}{\delta \psi_{N C}^{+}(x, t)} \frac{\delta}{\delta \psi_{C}(y, t)}\right\} \\
& \times \exp \left(i \int d x\left(\psi_{C}(x, t) \Xi_{C}^{+}(x, t)+\psi_{N C}(x, t) \Xi_{N C}^{+}(x, t)+\Xi_{C}(x, t) \psi_{C}^{+}(x, t)+\Xi_{N C}(x, t) \psi_{N C}^{+}(x, t)\right)\right. \\
& \times P\left[\underset{\psi}{\rightarrow}, \stackrel{\psi}{\psi}^{*}\right]
\end{aligned}
$$

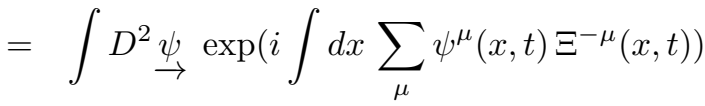

$$
\begin{aligned}
& \times \iint d x d y\left\{\frac{1}{2} \sum_{k \in C} \sum_{l \in N C} \phi_{N C l}^{+}(x, t) C_{C k N C l}^{-}(t) \phi_{C k}^{-}(y, t) \frac{\delta}{\delta \psi_{N C}^{+}(x, t)} \frac{\delta}{\delta \psi_{C}^{-}(y, t)} P\left[\underset{\rightarrow}{\rightarrow}, \psi^{*}\right]\right\}
\end{aligned}
$$

and

$$
\begin{aligned}
& \frac{1}{2}\left\{\iint d x d y\left\{\sum_{l \epsilon C} \sum_{k \in N C} \phi_{l}^{*}(x, t) C_{l k}^{*}(t) \phi_{k}(y, t)\right\}\left(i \Xi_{C}(x, t)\right)\left(i \Xi_{N C}^{+}(y, t)\right)\right\} \\
& \times \chi\left[\Xi_{C}, \Xi_{C}^{+}, \Xi_{N C}, \Xi_{N C}^{+}\right] \\
= & \int D^{2} \psi \frac{1}{\rightarrow} \iint d x d y\left\{\sum_{l \epsilon C} \sum_{k \in N C} \phi_{l}^{*}(x, t) C_{l k}^{*}(t) \phi_{k}(y, t)\right\}\left\{\frac{\delta}{\delta \psi_{C}^{+}(x, t)} \frac{\delta}{\delta \psi_{N C}(y, t)}\right\} \\
& \times \exp \left(i \int d x\left(\psi_{C}(x, t) \Xi_{C}^{+}(x, t)+\psi_{N C}(x, t) \Xi_{N C}^{+}(x, t)+\Xi_{C}(x, t) \psi_{C}^{+}(x, t)+\Xi_{N C}(x, t) \psi_{N C}^{+}(x, t)\right)\right. \\
& \times P\left[\underline{\psi}, \underline{\psi}^{*}\right] \\
= & \int D^{2} \underline{\psi} \rightarrow \exp \left(i \int d x \sum_{\mu} \psi^{\mu}(x, t) \Xi^{-\mu}(x, t)\right) \\
& \times \iint d x d y\left\{\frac{1}{2} \sum_{k \in N C} \sum_{l \epsilon C} \phi_{C l}^{+}(x, t) C_{C l N C k}^{+}(t) \phi_{N C k}^{-}(y, t) \frac{\delta}{\delta \psi_{C}^{+}(x, t)} \frac{\delta}{\delta \psi_{N C}^{-}(y, t)} P\left[\underline{\psi}_{\rightarrow}, \underline{\psi}^{*}\right]\right\}
\end{aligned}
$$

where functional integration by parts has been applied twice.

Equating both sides of $\frac{\partial}{\partial t} \chi\left[\Xi_{C}, \Xi_{C}^{+}, \Xi_{N C}, \Xi_{N C}^{+}\right]$via Eqs. (122), (127) and (128) gives the functional Fokker-Planck equation for the hybrid distribution function in the form 


$$
\begin{aligned}
& \frac{\partial}{\partial t} P\left[\stackrel{\psi}{\rightarrow}, \stackrel{\psi}{\rightarrow}^{*}\right] \\
& =\left\{-\sum_{\mu A} \int d x \frac{\delta}{\delta \psi_{A}^{\mu}(x, t)} A_{A}^{\mu}(x)+\frac{1}{2} \sum_{\mu A} \sum_{\nu B} \iint d x d y \frac{\delta}{\delta \psi_{A}^{\mu}(x, t)} \frac{\delta}{\delta \psi_{B}^{\nu}(y, t)} D_{A B}^{\mu \nu}(x, y)\right\} P\left[\underline{\psi}, \underline{\psi}_{\rightarrow}^{*}\right] \\
& +\frac{1}{2}\left\{\sum_{k \in C} \sum_{l \in N C} \iint d x d y \phi_{N C l}^{+}(x, t) C_{C k N C l}^{-}(t) \phi_{C k}^{-}(y, t) \frac{\delta}{\delta \psi_{N C}^{+}(x, t)} \frac{\delta}{\delta \psi_{C}^{-}(y, t)}\right\} P\left[\underline{\psi}, \underline{\psi}_{\rightarrow}^{*}\right] \\
& +\frac{1}{2}\left\{\sum_{k \in N C} \sum_{l \in C} \iint d x d y \phi_{C l}^{+}(x, t) C_{C l N C k}^{+}(t) \phi_{N C k}^{-}(y, t) \frac{\delta}{\delta \psi_{C}^{+}(x, t)} \frac{\delta}{\delta \psi_{N C}^{-}(y, t)}\right\} P\left[\underset{\psi}{\rightarrow}, \underline{\psi}^{*}\right] \\
& =\left\{-\sum_{\mu A} \int d x \frac{\delta}{\delta \psi_{A}^{\mu}(x, t)} A_{A}^{\mu}(x)+\frac{1}{2} \sum_{\mu A} \sum_{\nu B} \iint d x d y \frac{\delta}{\delta \psi_{A}^{\mu}(x, t)} \frac{\delta}{\delta \psi_{B}^{\nu}(y, t)} D_{A B}^{\mu \nu}(x, y)\right\} \\
& \times P\left[\stackrel{\psi}{\rightarrow}, \stackrel{\psi}{*}^{*}\right] \\
& +\frac{1}{2}\left\{\sum_{\mu A} \sum_{\nu B} \iint d x d y \frac{\delta}{\delta \psi_{A}^{\mu}(x, t)} \frac{\delta}{\delta \psi_{B}^{\nu}(y, t)}\left(\sum_{k} \sum_{l} \phi_{A k}^{\mu}(x, t) C_{A k B l}^{\mu}(t) \phi_{B l}^{\nu}(y, t)\right) \delta_{A C} \delta_{B N C} \delta_{\mu-\nu}\right\} \\
& \times P\left[\stackrel{\psi}{\rightarrow}, \stackrel{\psi}{*}^{*}\right]
\end{aligned}
$$




\section{Appendix - Derivation of Ito Stochastic Field Equations}

In this Appendix details for the derivation of the Ito stochastic field equations in Section 3 are set out.

\subsection{Derivative of Phase Space Functional Integral}

To evaluate the first term involving $\frac{\partial}{\partial t} F[\underline{\psi}]$ we first consider changes $\delta \psi_{A}^{\mu}(x, t)$ in $\psi_{A}^{\mu}(x, t)$ during an interval $\delta t$ that results in a change $\delta F[\underset{\psi}{\rightarrow}]$ to the functional $F[\underline{\psi}]$. From Eq.(182) we have

$$
\begin{aligned}
F[\underline{\psi}+\delta \underline{\psi}]-F[\underline{\psi}] & \doteqdot \sum_{\mu A} \int d x\left(\frac{\delta}{\delta \psi_{A}^{\mu}(x, t)} F[\underline{\psi}]\right) \delta \psi_{A}^{\mu}(x, t) \\
\delta F[\underline{\psi}] & \doteqdot \sum_{\mu A} \int d x\left(\frac{\delta}{\delta \psi_{A}^{\mu}(x, t)} F[\underline{\psi}]\right) \frac{\partial \psi_{A}^{\mu}(x, t)}{\partial t} \delta t \\
\frac{\partial}{\partial t} F[\underline{\psi}] & =\sum_{\mu A} \int d x\left(\frac{\delta}{\delta \psi_{A}^{\mu}(x, t)} F[\underline{\psi}]\right) \frac{\partial \psi_{A}^{\mu}(x, t)}{\partial t}
\end{aligned}
$$

Hence the first term in (132) is given by

$$
\begin{aligned}
& \left.\int D^{2} \underset{\psi}{\rightarrow} \frac{\partial}{\partial t} F[\underset{\psi}{\rightarrow}] \stackrel{\underline{\psi}}{\rightarrow}, \underline{\psi}^{*}\right] \\
& =\int D^{2} \underset{\rightarrow}{\rightarrow} \sum_{\mu A} \int d x\left(\frac{\delta}{\delta \psi_{A}^{\mu}(x, t)} F[\underline{\psi}]\right) \\
& \times\left\{\int d y \sum_{B}\left(\sum_{k l} \phi_{A k}^{\mu}(x, t) C_{A k B l}^{\mu} \phi_{B l}^{-\mu}(y, t)\right) \psi_{B}^{\mu}(y, t)\right\} P[\underset{\rightarrow}{\rightarrow}, \underset{\rightarrow}{*}] \\
& +\int D^{2} \underset{\psi}{\rightarrow} \sum_{\mu A} \int d x\left(\frac{\delta}{\delta \psi_{A}^{\mu}(x, t)} F[\underset{\psi}{\rightarrow}]\right) \\
& \left.\times\left\{\int d y \sum_{B}\left(\sum_{k l} \phi_{B l}^{\mu}(x, t) C_{A k B l}^{-\mu} \phi_{A k}^{-\mu}(y, t)\right) \psi_{A}^{\mu}(y, t)\right\} P \underset{\rightarrow}{\rightarrow}, \underset{\rightarrow}{\rightarrow}\right] \\
& =\left\langle\sum_{\mu A} \int d x\left(\frac{\delta}{\delta \psi_{A}^{\mu}(x, t)} F[\underset{\rightarrow}{\rightarrow})\left\{\int d y \sum_{B}\left(\sum_{k l} \phi_{A k}^{\mu}(x, t) C_{A k B l}^{\mu} \phi_{B l}^{-\mu}(y, t)\right) \psi_{B}^{\mu}(y, t)\right\}\right\rangle\right. \\
& +\left\langle\sum_{\mu A} \int d x\left(\frac{\delta}{\delta \psi_{A}^{\mu}(x, t)} F[\underset{\rightarrow}{\rightarrow})\left\{\int d y \sum_{B}\left(\sum_{k l} \phi_{B l}^{\mu}(x, t) C_{A k B l}^{-\mu} \phi_{A k}^{-\mu}(y, t)\right) \psi_{A}^{\mu}(y, t)\right\}\right\rangle\right.
\end{aligned}
$$

Using the functional Fokker-Planck equation (129) we find after using inte- 
gration by parts that the second term in (132) is

$$
\begin{aligned}
& \int D^{2} \underset{\rightarrow}{\rightarrow} F\left[\underset{\rightarrow}{\rightarrow} \frac{\partial}{\partial t} P\left[\stackrel{\psi}{\rightarrow}, \underline{\psi}^{*}\right]\right. \\
& =\int D^{2} \underset{\rightarrow}{\rightarrow}\left\{\sum_{\mu A} \int d x\left(\frac{\delta}{\delta \psi_{A}^{\mu}(x, t)} F[\underline{\psi}]\right) A_{A}^{\mu}(x)\right\} P\left[\underset{\rightarrow}{\rightarrow}, \underline{\psi}^{*}\right] \\
& +\int D^{2} \underset{\rightarrow}{\rightarrow}\left\{\frac{1}{2} \sum_{\mu A} \sum_{\nu B} \iint d x d y\left(\frac{\delta}{\delta \psi_{A}^{\mu}(x, t)} \frac{\delta}{\delta \psi_{B}^{\nu}(y, t)} F[\underline{\psi}]\right) E_{A B}^{\mu \nu}(x, y)\right\} P\left[\underline{\psi}, \underline{\psi}_{\rightarrow}^{*}\right] \\
& =\left\langle\sum_{\mu A} \int d x\left(\frac{\delta}{\delta \psi_{A}^{\mu}(x, t)} F[\underset{\rightarrow}{\rightarrow}) A_{A}^{\mu}(x)\right\rangle\right. \\
& +\left\langle\frac{1}{2} \sum_{\mu A} \sum_{\nu B} \iint d x d y\left(\frac{\delta}{\delta \psi_{A}^{\mu}(x, t)} \frac{\delta}{\delta \psi_{B}^{\nu}(y, t)} F[\underset{\psi}{\rightarrow}]\right) E_{A B}^{\mu \nu}(x, y)\right\rangle
\end{aligned}
$$

where functional integration by parts has been used.

\subsection{Derivative of Stochastic Field Averages}

For the first order derivative terms we have on substituting from (139) and expanding

$$
\begin{aligned}
& \overline{\int d x\left\{\sum_{\mu A} \delta \psi_{A}^{\mu s}(x, t)\left(\frac{\delta F\left[\psi^{s}, \psi^{s+}\right]}{\delta \psi_{A}^{\mu s}(x, t)}\right)_{x}\right\}} \\
= & \frac{\sqrt{d x \sum_{\mu A}\left(\frac{\delta F\left[\psi^{s}, \psi^{s+}\right]}{\delta \psi_{A}^{\mu s}(x, t)}\right)_{x} \mathcal{G}_{A}^{\mu}(x)} \delta t}{} \\
& +\frac{\int d x \sum_{\mu A}\left(\frac{\delta F\left[\psi^{s}, \psi^{s+}\right]}{\delta \psi_{A}^{\mu s}(x, t)}\right)_{x} \sum_{a} \mathcal{N}_{A a}^{\mu}(x)}{\int_{t}^{t+\delta t} d t_{1} \Gamma_{a}\left(t_{1}\right)} \\
= & \int d x \sum_{\mu A}\left(\frac{\delta F\left[\psi^{s}, \psi^{s+}\right]}{\delta \psi_{A}^{\mu s}(x, t)}\right)_{x} \mathcal{G}_{A}^{\mu}(x) \delta t
\end{aligned}
$$

where the stochastic average rules for sums and products have been used, the non-correlation between the averages of functions of $\psi_{\mu}^{s}(x, t)$ at time $t$ and the $\Gamma$ at later times between $t$ to $t+\delta t$ is applied and the term involving $\overline{\Gamma_{a}\left(t_{1}\right)}$ is equal to zero from (78). Note that this term is proportional to $\delta t$.

For the second order derivative terms we have on substituting from (139) 
and expanding we find that

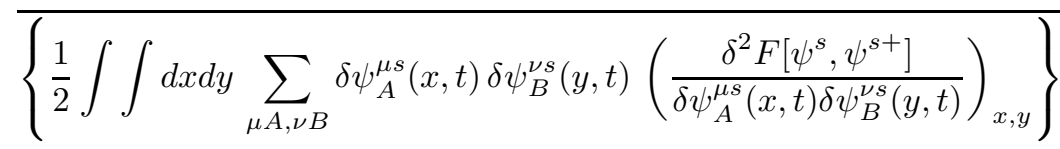

$$
\begin{aligned}
& =\overline{\frac{1}{2} \iint d x d y \sum_{\mu A, \nu B}\left(\frac{\delta^{2} F\left[\psi^{s}, \psi^{s+}\right]}{\delta \psi_{A}^{\mu s}(x, t) \delta \psi_{B}^{\nu s}(y, t)}\right)_{x, y}\left[\mathcal{G}_{A}^{\mu}(x) \mathcal{G}_{B}^{\nu}(y)\right]} \delta t^{2} \\
& +\overline{\frac{1}{2} \iint d x d y \sum_{\mu A, \nu B}\left(\frac{\delta^{2} F\left[\psi^{s}, \psi^{s+}\right]}{\delta \psi_{A}^{\mu S}(x, t) \delta \psi_{B}^{\nu S}(y, t)}\right)_{x, y} \mathcal{G}_{A}^{\mu}(x) \delta t \sum_{b} \mathcal{N}_{B b}^{\nu}(y)} \\
& \times \overline{\int_{t}^{t+\delta t} d t_{2} \Gamma_{b}\left(t_{2}\right)} \delta t \\
& +\overline{\frac{1}{2} \iint d x d y \sum_{\mu A, \nu B}\left(\frac{\delta^{2} F\left[\psi^{s}, \psi^{s+}\right]}{\delta \psi_{A}^{\mu s}(x, t) \delta \psi_{B}^{\nu s}(y, t)}\right)_{x, y}\left[\sum_{a} \mathcal{N}_{A a}^{\mu}(x) \mathcal{G}_{B}^{\nu}(y) \delta t\right]} \\
& \times \overline{\int_{t}^{t+\delta t} d t_{1} \Gamma_{a}\left(t_{1}\right)} \delta t \\
& +\overline{\frac{1}{2} \iint d x d y \sum_{\mu A, \nu B}\left(\frac{\delta^{2} F\left[\psi^{s}, \psi^{s+}\right]}{\delta \psi_{A}^{\mu s}(x, t) \delta \psi_{B}^{\nu s}(y, t)}\right)_{x, y}\left[\sum_{a} \mathcal{N}_{A a}^{\mu}(x) \sum_{b} \mathcal{N}_{B b}^{\nu}(y)\right]} \\
& \times \overline{\int_{t}^{t+\delta t} d t_{1} \Gamma_{a}\left(t_{1}\right) \int_{t}^{t+\delta t} d t_{2} \Gamma_{b}\left(t_{2}\right)}
\end{aligned}
$$

where the stochastic average rules for sums and products have been used, the non-correlation between the averages of functions of $\psi_{\mu}^{s}(x, t)$ at time $t$ and the $\Gamma$ at later times between $t$ to $t+\delta t$ is applied. The terms involving a single $\Gamma$ have a zero stochastic average, whilst from Eq.(78) the terms with two $\Gamma$ give a stochastic average proportional to $\delta t$

$$
\begin{aligned}
\overline{\int_{t}^{t+\delta t} d t_{1} \Gamma_{a}\left(t_{1}\right) \int_{t}^{t+\delta t} d t_{2} \Gamma_{b}\left(t_{2}\right)} & =\int_{t}^{t+\delta t} d t_{1} \int_{t}^{t+\delta t} d t_{2} \overline{\Gamma_{a}\left(t_{1}\right) \Gamma_{b}\left(t_{2}\right)} \\
& =\int_{t}^{t+\delta t} d t_{1} \int_{t}^{t+\delta t} d t_{2} \delta_{a b} \delta\left(t_{1}-t_{2}\right) \\
& =\delta_{a b} \delta t
\end{aligned}
$$


so that correct to order $\delta t$ the second order derivative term is

$$
\begin{aligned}
& \left\{\frac{1}{2} \iint d x d y \sum_{\mu A, \nu B} \delta \psi_{A}^{\mu s}(x, t) \delta \psi_{B}^{\nu s}(y, t)\left(\frac{\delta^{2} F\left[\psi^{s}, \psi^{s+}\right]}{\delta \psi_{A}^{\mu s}(x, t) \delta \psi_{B}^{\nu s}(y, t)}\right)_{x, y}\right\} \\
& =\overline{\frac{1}{2} \iint d x d y \sum_{\mu A, \nu B}\left(\frac{\delta^{2} F\left[\psi^{s}, \psi^{s+}\right]}{\delta \psi_{A}^{\mu s}(x, t) \delta \psi_{B}^{\nu s}(y, t)}\right)_{x, y}\left[\sum_{a} \mathcal{N}_{A a}^{\mu}(x) \mathcal{N}_{B a}^{\nu}(y)\right]} \delta t \\
& =\overline{\frac{1}{2} \iint d x d y \sum_{\mu A, \nu B}\left(\frac{\delta^{2} F\left[\psi^{s}, \psi^{s+}\right]}{\delta \psi_{A}^{\mu s}(x, t) \delta \psi_{B}^{\nu S}(y, t)}\right)_{x, y}\left[\left[\mathcal{N}(x) \mathcal{N}^{T}(y)\right]_{A, B}^{\mu, \nu}\right]} \delta t
\end{aligned}
$$

